TRANSACTIONS OF THE

AMERICAN MATHEMATICAL SOCIETY

Volume 354, Number 2, Pages 427-490

S 0002-9947(01)02885-9

Article electronically published on September 26, 2001

\title{
A NON-HOMOGENEOUS BOUNDARY-VALUE PROBLEM FOR THE KORTEWEG-DE VRIES EQUATION IN A QUARTER PLANE
}

\author{
JERRY L. BONA, S. M. SUN, AND BING-YU ZHANG
}

\begin{abstract}
The Korteweg-de Vries equation was first derived by Boussinesq and Korteweg and de Vries as a model for long-crested small-amplitude long waves propagating on the surface of water. The same partial differential equation has since arisen as a model for unidirectional propagation of waves in a variety of physical systems. In mathematical studies, consideration has been given principally to pure initial-value problems where the wave profile is imagined to be determined everywhere at a given instant of time and the corresponding solution models the further wave motion. The practical, quantitative use of the Korteweg-de Vries equation and its relatives does not always involve the pure initial-value problem. Instead, initial-boundary-value problems often come to the fore. A natural example arises when modeling the effect in a channel of a wave maker mounted at one end, or in modeling near-shore zone motions generated by waves propagating from deep water. Indeed, the initial-boundary-value problem
\end{abstract}

$$
\left\{\begin{array}{l}
\eta_{t}+\eta_{x}+\eta \eta_{x}+\eta_{x x x}=0, \quad \text { for } x, t \geq 0, \\
\eta(x, 0)=\phi(x), \quad \eta(0, t)=h(t),
\end{array}\right.
$$

studied here arises naturally as a model whenever waves determined at an entry point propagate into a patch of a medium for which disturbances are governed approximately by the Korteweg-de Vries equation. The present essay improves upon earlier work on (0.1) by making use of modern methods for the study of nonlinear dispersive wave equations. Speaking technically, local wellposedness is obtained for initial data $\phi$ in the class $H^{s}\left(R^{+}\right)$for $s>\frac{3}{4}$ and boundary data $h$ in $H_{l o c}^{(1+s) / 3}\left(R^{+}\right)$, whereas global well-posedness is shown to hold for $\phi \in H^{s}\left(R^{+}\right), h \in H_{l o c}^{\frac{7+3 s}{12}}\left(R^{+}\right)$when $1 \leq s \leq 3$, and for $\phi \in$ $H^{s}\left(R^{+}\right), h \in H_{\text {loc }}^{(s+1) / 3}\left(R^{+}\right)$when $s \geq 3$. In addition, it is shown that the correspondence that associates to initial data $\phi$ and boundary data $h$ the unique solution $u$ of $(0.1)$ is analytic. This implies, for example, that solutions may be approximated arbitrarily well by solving a finite number of linear problems.

Received by the editors April 19, 2000 and, in revised form, January 8, 2001.

2000 Mathematics Subject Classification. Primary 35Q53; Secondary 76B03, 76B15.

Key words and phrases. Korteweg-de Vries equation, KdV equation in a quarter plane, nonhomogeneous problems, well-posedness.

JLB was partially supported by the National Science Foundation and by the W. M. Keck Foundation.

SMS was partially supported by National Science Foundation grant DMS-9971764.

BYZ was partially supported by a Taft Competitive Faculty Fellowship. Part of the work was done while BYZ was a Research Fellow of the Texas Institute for Computational and Applied Mathematics at the University of Texas at Austin.

The line of argument in Section 3 reflects a very helpful suggestion by a referee, for which the authors are grateful. 


\section{INTRODUCTION}

This paper is concerned with the wave maker problem for the classical Kortewegde Vries equation. In this conception, it is imagined that water at rest in a channel is set in motion by a wave maker mounted at one end of the channel. If the frequency and amplitude of the wave maker oscillations are appropriately restricted, this will generate small-amplitude long waves that propagate down the channel, and thus will be brought into being motion that corresponds more or less exactly to the Korteweg-de Vries regime. Indeed, the amplitude of the wave maker is related to the amplitude of the generated waves, while the frequency of the wave maker is related inversely to the wavelength. In this situation, the most convenient and accurate measurements that can be made are to monitor the free surface at fixed points down the channel from the wave maker. This scheme has been followed in a number of experimental works (cf. Zabusky and Galvin 62, Hammack 32, Hammack and Segur [33] and Bona, Pritchard and Scott [7]). Such a physical configuration is naturally modeled by the initial-boundary-value problem

$$
\left\{\begin{array}{l}
\eta_{t}+\eta_{x}+\eta \eta_{x}+\eta_{x x x}=0, \quad \text { for } x, t \geq 0 \\
\eta(x, 0)=0, \quad \eta(0, t)=h(t)
\end{array}\right.
$$

where $x$ is proportional to distance along the channel with $x=0$ corresponding to the point closest to the wave maker where measurements are taken, $t$ is proportional to elapsed time with $t=0$ being the initial time when the water surface is quiescent and the wave maker is activated, and $\eta(x, t)$ is proportional to the deviation of the free surface at the point $x$ down the channel at time $t$ (see [7, 10] for more detailed commentary on this modeling stance). For each relevant time $t$, the value $h(t)$ is the measured deviation of the free surface from its rest position at the point $x=0$ at time $t$. The function $h$ acts as the driving force for the mathematical problem (1.1).

Several points are worth noting about the modeling inherent in (1.1). First, the perfect fluid assumption that leads to the Korteweg-de Vries equation has not been relaxed. Dissipative effects need to be taken into account in any practical use of this model, but they are ignored in the present analysis. Second, the channel has been assumed to extend infinitely away from the wave maker. This corresponds to ignoring wave reflection from the end of the channel or from a beach. In practice, this will mean either that the beach is very gently sloping so that little energy does in fact come back, or, in a channel, the experiment takes place over a time scale such that the wave motion does not reach the end of the channel. In any event, if there is significant reflected wave motion moving back toward the wave maker, this model is inappropriate, as one of its hallmarks is unidirectionality of propagation. (To take account of two-way propagation at the KdV level of approximation, a Boussinesq system of equations would be needed as in [3, 4 for example.) Furthermore, notice that the usual caveat where one removes the term $\eta_{x}$ from the equation by changing to traveling coordinates is not available without a real price in the quarter-plane problem. A change of variables where one lets $v(x, t)=\eta(x+t, t)$ does indeed dispense with the offending term in the evolution equation, but the boundary condition must now be applied in the form $v(-t, t)=h(t)$ for $t \geq 0$. Thus the boundary condition is applied at a changing spatial point and the problem is posed in the peculiar domain $\{(x, t): t \geq 0, x+t \geq 0\}$, rather than a quarter plane. The gain 
in simplicity of the equation does not appear to justify the difficulty caused by the application of a boundary condition at a moving point, and therefore we have elected to stay in laboratory coordinates as expressed in (1.1). If one drops the term $\eta_{x}$ arbitrarily, the resulting initial-boundary-value problem may be treated by a considerably simplified version of the analysis that is developed here for (1.1).

Analogous considerations apply to other physical situations modeled approximately by the Korteweg-de Vries equation, and lead also to the problem (1.1).

The problem (1.1) has received attention in the past, and a satisfactory theory exists corresponding to physically relevant smoothness assumptions on the initial and boundary data (cf. [6, 10, 11, 22, 23]). In fact, the problem is usually posed with allowance made for a more general initial configuration, thus in the form

$$
\left\{\begin{array}{l}
\eta_{t}+\eta_{x}+\eta \eta_{x}+\eta_{x x x}=0, \quad \text { for } x, t \geq 0, \\
\eta(x, 0)=\phi(x), \quad \eta(0, t)=h(t) .
\end{array}\right.
$$

Naturally, the consistency condition $\phi(0)=h(0)$ is imposed on the auxiliary data. Global well-posedness results for strong solutions up to the boundary were established in 10. 11 for suitably smooth $\phi$ and $h$ that satisfy certain compatibility conditions. Included in the theory is the continuity of the mapping that associates to given initial- and boundary-data the corresponding solution of (1.2). Faminskii, in a wide-ranging paper [23], deals with the initial-boundary-value problem (1.2) for a generalization of the $\mathrm{KdV}$ equation somewhat like that appearing later in Craig, Kappeler and Strauss [20]. He puts forward a theory of well-posedness for generalized solutions set in weighted $H^{1}$-Sobolev classes. Moreover, he obtains extra interior regularity in case the initial data decays suitably rapidly at $+\infty$. The program of Fokas, Its and Pelloni [24, 25, 26, 27] whereby the inverse-scattering transform on $R$ is adapted to $R^{+}$also deserves notice. This method yields very interesting and helpful formal long-time asymptotics, and it seems likely it will also be useful in further, detailed studies of the nonlinear problem. We also point to related work on the periodic- and two-point-boundary-value problem for the KdV equation posed on a finite interval (see [15], [49], [56], [63], [18]).

By contrast, the mathematical theory pertaining to the pure initial-value problem for the $\mathrm{KdV}$ equation posed on the whole real line $R$ or on a finite interval with periodic boundary conditions is considerably more advanced. Before recent developments, the problem

$$
\eta_{t}+\eta \eta_{x}+\eta_{x x x}=0, \quad \eta(x, 0)=\phi(x),
$$

posed for $x \in R$ or over a finite interval with periodic boundary conditions, was known to be locally well-posed in the space $H^{s}(R)$ of square-integrable functions whose first $s$ derivatives are also square integrable, for $s>3 / 2$ and globally wellposed in the same space if $s \geq 2$ (see [9, 35, 36, 37, 38]). Various types of weak solutions were also known to exist. These results were obtained by studying a corresponding regularized equation, by applying general abstract semigroup theory and by other methods of nonlinear functional analysis [9, 21, 34, 35, 51, 53, 58, As remarked already by Saut and Temam [52], solving the initial-value problem (1.3) cannot result in the solution being more regular in the $H^{s}$-spaces than it is initially, because the equation is time reversible. Thus, there is no smoothing associated with solving the initial-value problem (1.3) of the sort that obtains when one solves the linear heat equation or Burger's equation, for example. 
There is, however, more subtle smoothing associated with the initial-value problem (1.3). One of the early expressions of this fact appears in the papers of Cohen [16, 17] and, later, Sachs [50. These works made use of the inverse-scattering representation of solutions. The outcome is a set of results showing that decay of initial data at $+\infty$ translates into a local smoothing of the solution beyond that which it has initially. Starting late in the 1970's, those smoothing properties were investigated by techniques other than the inverse-scattering representation of solutions. Kato [37, 38, and independently, Kruzhkov and Faminskii [45, 46] realized among other things that solutions of (1.3) whose initial data lies in $H^{s}(R)$ not only lie in $C\left(0, T ; H^{s}(R)\right)$, but also in $L^{2}\left(0, T ; H_{l o c}^{s+1}(R)\right)$. This property, now known as Kato-smoothing, stimulated an extensive investigation of various smoothing properties of the $\mathrm{KdV}$-equation and other dispersive wave equations (see, for example, 13, 14, 19, 20, 30, 39, 40, 41, 42, 43, 44, 54, 59, 61 and the references therein). In particular, various Strichartz-type $L_{t}^{p}-L_{x}^{q}$ estimates were established for the linear problem

$$
\eta_{t}+\eta_{x x x}=0, \quad \eta(x, 0)=\phi(x),
$$

associated to (1.3) in the work of Kenig-Ponce-Vega [39]- 44] and Bourgain [13, 14]. Those linear estimates made it possible to apply the contraction-mapping principle to establish directly the well-posedness of the pure initial-value problem (1.3) for functions $\phi$ defined on $R$ which either decay to zero at infinity or which are periodic. Indeed, the problem (1.3) posed on the whole line $R$ was shown to be well-posed in the space $H^{s}(R)$ for $s>\frac{3}{4}$ by Kenig, Ponce and Vega 41], and in larger spaces by Bourgain [14] and Kenig, Ponce and Vega [43, 44, culminating in a well-posedness result set in $H^{s}(R)$ for any $s>-\frac{3}{4}$. As for the initial-value problem (1.3) posed on a periodic domain $S$, it was proved to be well-posed in $H^{s}(S)$ for $s \geq 0$ by Bourgain [14] and for $s>-1 / 2$ by Kenig, Ponce and Vega [44]. (Henceforth, the abbreviation "IVP" will stand for the often-used phrase "initial-value problem" while the mnemonic "IBVP" stands for "initial-boundary-value problem".)

Because of well-posedness, the IVP (1.3) defines a nonlinear map $K_{H}$ from the space $H^{s}$ to $C\left([0, T] ; H^{s}\right)\left(H^{s}\right.$ stands for $H^{s}(R)$ or $H^{s}(S)$ depending on whether (1.3) is considered on $R$ or on $S$ ). This map was shown to be continuous from $H^{s}$ to $C\left([0, T] ; H^{s}\right)$ by Bona and Smith [9] and Kato [36], and Hölder continuous with exponent $1 / 2$ from the space $H^{s+1 / 2}$ to the space $L^{\infty}\left(0, T ; H^{s}\right)$ by Saut and Temam [52]. Much stronger regularity can be established by taking advantage of the smoothing properties of the equation. Simply as a by-product of their contractionprinciple approach to the IVP (1.3), Kenig, Ponce and Vega [42] obtained that the map $K_{H}$ is Lipschitz continuous from the space $H^{s}$ to the space $C\left([0, T] ; H^{s}\right)$. Later, based on the previously mentioned works of Kenig, Ponce, Vega, and Bourgain, Zhang [64, 65, 66] proved that the map $K_{H}$ is infinitely Fréchet differentiable from the space $H^{s}$ to the space $C\left([0, T] ; H^{s}\right)$ and that for $\delta>0$ sufficiently small, the formal Taylor series expansion

$$
K_{H}(\phi+\psi)=\sum_{n=0}^{\infty} \frac{K_{H}^{(n)}(\phi)\left[\psi^{n}\right]}{n !}
$$

converges in $C\left([0, T] ; H^{s}\right)$ uniformly for $\|\psi\|_{s} \leq \delta$, which is the same as saying that the map $K_{H}$ is analytic from the space $H^{s}$ to the space $C\left([0, T] ; H^{s}\right)$. Here, $K_{H}^{(n)}(\phi)$ is the $n$-th derivative of $K_{H}$ at $\phi$, an $n$-multilinear map from the $n$-fold 
product of $H^{s}$ with itself to $C\left([0, T] ; H^{s}\right)$. Consequently, the solution of the IVP (1.3) may be approximated arbitrarily well on a finite time interval by solving a finite number of linear problems.

Our purpose in this paper is to bring the theory for the quarter-plane problem (1.2) at least partly into line with the modern theory for the pure initial-value problem (1.3) posed on $R$. The following results will be established in this paper.

The initial-boundary-value problem (1.2) is locally well-posed for initial data $\phi$ in the space $H^{s}\left(R^{+}\right)$and boundary data $h$ in the space $H_{l o c}^{(s+1) / 3}\left(R^{+}\right)$satisfying certain compatibility conditions (see Section 4 ) for $s>3 / 4$, whereas global well-posedness holds for $\phi \in H^{s}\left(R^{+}\right), h \in H^{\frac{7+3 s}{12}}\left(R^{+}\right)$when $1 \leq s \leq 3$ and for $\phi \in H^{s}\left(R^{+}\right)$, $h \in H_{\text {loc }}^{(s+1) / 3}\left(R^{+}\right)$when $s \geq 3$. Furthermore, the corresponding solution map is an analytic correspondence between the space of initial- and boundary-data and the solution space.

The crux of the modern analysis of nonlinear, dispersive evolution equations is the linear estimates to which reference was made above. For the IBVP (1.2) under consideration here, the associated linear problem

$$
\begin{cases}\eta_{t}+\eta_{x}+\eta_{x x x}=0, & \text { for } x, t \geq 0 \\ \eta(x, 0)=\phi(x), & \eta(0, t)=h(t)\end{cases}
$$

plays the same central role that the linearized IVP

$$
\eta_{t}+\eta_{x x x}=0, \quad \eta(x, 0)=\phi(x),
$$

does in the study of (1.3). Since (1.6) is posed in a quarter plane, the Fourier transform does not possess the same power it has when the problem is presented on all of $R^{2}$. As a potential global solution of (1.6) is defined on a half-line $R^{+}$in each of the two independent variables $x$ and $t$, it is not unnatural to think of replacing the use of the Fourier transform that comes to the fore in the analysis of (1.7) with the Laplace transform. By taking the Laplace transform with respect to $t$ of both sides of the equation in (1.6), the IBVP is converted to a one-parameter family of third-order, boundary-value problems

$$
\left\{\begin{array}{l}
\lambda \hat{\eta}(x, \lambda)+\hat{\eta}_{x}(x, \lambda)+\hat{\eta}_{x x x}(x, \lambda)=\phi(x) \\
\hat{\eta}(0, \lambda)=\hat{h}(\lambda), \quad \hat{\eta}(+\infty, \lambda)=0, \quad \hat{\eta}_{x}(+\infty, \lambda)=0
\end{array}\right.
$$

where $\hat{\eta}=\hat{\eta}(x, \lambda)$ denotes the Laplace transform of $\eta=\eta(x, t)$ with respect to $t$ and $\lambda>0$ is the dual variable. The solution of (1.8) is given by

$$
\hat{\eta}(x, \lambda)=\int_{0}^{+\infty} G\left(x, x_{0} ; \lambda\right) \phi\left(x_{0}\right) d x_{0}+e^{r(\lambda) x} \hat{h}(\lambda)
$$

where $G\left(x, x_{0} ; \lambda\right)$ is the Green's function associated with (1.8) in the special case wherein $\hat{h} \equiv 0$ and $r(\lambda)$ is the solution of

$$
\lambda+r+r^{3}=0
$$

for which $\operatorname{Re} r(\lambda)<0$. The solution $\eta$ of 1.6 is then given formally by

$$
\eta(x, t)=\frac{1}{2 \pi i} \int_{-\infty+i \gamma}^{+\infty+i \gamma} e^{\lambda t}\left(\int_{0}^{+\infty} G\left(x, x_{0} ; \lambda\right) \phi\left(x_{0}\right) d x_{0}+e^{r(\lambda) x} \hat{h}(\lambda)\right) d \lambda
$$


for $x, t \geq 0$, where $\gamma$ is a fixed, but arbitrary positive constant. At first sight, a solution formula like this does not appear well suited to analysis by Fourier techniques. However, by carefully choosing the contour over which the outer integral is computed, we are able to rewrite the representation in (1.9) to obtain the following, remarkable explicit formula. Define $H=H(x, t)$ by

$$
\left\{\begin{array}{l}
H(x, t)=\frac{1}{2 \pi} \int_{1}^{\infty} e^{i \mu^{3} t-i \mu t} \int_{0}^{\infty} e^{i \mu(x-\xi)} \phi(\xi) d \xi d \mu \\
+\frac{1}{2 \pi} \int_{1}^{\infty} e^{i \mu^{3} t-i \mu t} e^{-\frac{i \mu+\sqrt{3 \mu^{2}-4}}{2} x} \int_{0}^{\infty} e^{-i \mu \xi} \phi(\xi) d \xi d \mu \\
+\frac{1}{2 \pi i} \int_{0}^{\infty} e^{-\mu^{3} t-\mu t} e^{-\frac{\mu-i \sqrt{4+3 \mu^{2}}}{2} x} \int_{0}^{\infty} e^{-\mu \xi} \phi(\xi) d \xi d \mu \\
+\frac{1}{2 \pi} \int_{1}^{\infty} e^{i \mu^{3} t-i \mu t} e^{-\frac{\sqrt{\mu^{2}-4}+i \mu}{2} x}\left(3 \mu^{2}-1\right) \int_{0}^{\infty} e^{-i \mu^{3} \xi+i \mu \xi} h(\xi) d \xi d \mu .
\end{array}\right.
$$

In terms of $H$, the solution $\eta$ of (1.6) is, for $x, t \geq 0$,

$$
\eta(x, t)=H(x, t)+\overline{H(x, t)} .
$$

A cursory examination of (1.10)-(1.11) reveals that there are two types of smoothing properties associated with the linear problem (1.6); the smoothing effected upon $\eta$ with respect to its initial data $\phi$ and with regard to its boundary data $h$. As in the work on the IVP (1.3), these aspects are the heart of the theory to be developed here. It will be shown that for $h \equiv 0$, the solution $\eta$ of (1.6) satisfies the following estimates: For $\phi \in L_{2}\left(R^{+}\right)$,

$$
\begin{array}{r}
\sup _{0 \leq x<+\infty} \int_{0}^{\infty}\left|\partial_{x} \eta(x, t)\right|^{2} d t \leq C\|\phi\|_{L^{2}\left(R^{+}\right)}, \\
\sup _{0 \leq x<\infty}\|\eta(x, \cdot)\|_{H_{t}^{1 / 3}\left(R^{+}\right)} \leq C\|\phi\|_{L^{2}\left(R^{+}\right)},
\end{array}
$$

for $\phi \in H^{1 / 2}\left(R^{+}\right)$,

$$
\left(\int_{0}^{+\infty} \sup _{0 \leq x<\infty}\left|\partial_{x} \eta(x, t)\right|^{4} d t\right)^{1 / 4} \leq C\|\phi\|_{H^{1 / 2}\left(R^{+}\right)}
$$

whereas, for $s>\frac{3}{4}$ and $\phi \in H_{0}^{s}\left(R^{+}\right)$,

$$
\left(\int_{0}^{+\infty} \sup _{0 \leq t \leq T}|\eta(x, t)|^{2} d x\right)^{1 / 2} \leq C(1+T)\|\phi\|_{H^{s}\left(R^{+}\right)} .
$$

On the other hand, if $\phi \equiv 0$ and $h \in H^{1 / 3}\left(R^{+}\right)$, the solution $\eta$ of (1.6) will be shown to satisfy the following inequalities:

$$
\begin{aligned}
\sup _{0 \leq t<\infty}\|\eta(\cdot, t)\|_{L^{2}\left(R^{+}\right)} & \leq C\|h\|_{H^{1 / 3}\left(R^{+}\right)}, \\
\left(\int_{0}^{+\infty} \sup _{0 \leq t<\infty}|\eta(x, t)|^{2} d x\right) & \leq C\|h\|_{H^{1 / 3}\left(R^{+}\right)}
\end{aligned}
$$




$$
\begin{aligned}
\sup _{0 \leq x<\infty} & \left(\int_{0}^{+\infty}\left|\partial_{x} \eta(x, t)\right|^{2} d t\right)^{1 / 2} \leq C\|h\|_{H^{1 / 3}\left(R^{+}\right)}, \\
& \sup _{0 \leq x<\infty}\|\eta(x, \cdot)\|_{H_{t}^{1 / 3}\left(R^{+}\right)} \leq C\|h\|_{H^{1 / 3}\left(R^{+}\right)},
\end{aligned}
$$

and if $h \in H^{1 / 2}\left(R^{+}\right)$, then

$$
\left(\int_{0}^{+\infty} \sup _{0 \leq x<\infty}\left|\partial_{x} \eta(x, t)\right| d t\right)^{1 / 4} \leq C\|h\|_{H^{1 / 2}\left(R^{+}\right)} .
$$

The estimates (1.12) and (1.18) are sharp versions of the local smoothing effect of Kato-type. The estimates (1.14) and (1.20) reveal global smoothing effects of Strichartz-type for the linear problem (1.6). The estimates (1.15) and (1.17) are half-line versions of Corollary 2.9 in [41]. The estimates (1.13) and (1.19) comprise sharp trace results.

Armed with these linear estimates, we are able to obtain local well-posedness results for the IBVP (1.2) via the contraction-mapping principle. In our initial foray, we approached the linear estimates directly by way of the representation (1.10)-(1.11). One of the referees remarked that the estimates pertaining to the solution $\eta$ of (1.6) with $h \equiv 0$ can be reduced to the problem where $\phi \equiv 0$ together with the known results for the initial-value problem

$$
u_{t}+u_{x}+u_{x x x}=0, \quad u(x, 0)=\phi^{*}(x),
$$

for $x \in R, t \geq 0$, where $\phi^{*}(x)$ is a suitable extension of the initial data $\phi$ defined on the half-line $R^{+}$to the entire real axis. Indeed, by letting $v(x, t)=u(x, t)-\eta(x, t)$, one obtains a function defined on $R^{+} \times R^{+}$satisfying the linear KdV equation and with $v(0, t)=u(0, t)$ and $v(x, 0) \equiv 0$. In our development, we have taken advantage of this suggestion, thereby saving quite a few pages of detailed inequalities. On the other hand, we have kept the derivation of the exact solution (1.10)-(1.11) despite the fact that we need only a portion of this formula. Exact formulas are often useful when more detailed aspects of solutions are examined.

Global well-posedness follows from the local theory as soon as corresponding global a priori estimates are established. In contrast to the pure initial-value problem (1.3), where the needed global estimates are provided with little effort by the infinitely many conservation laws possessed by the IVP for the KdV equation, more protracted analysis is demanded in establishing global a priori estimates for the IBVP (1.2) because these conservation laws no longer hold.

In [10], using energy estimates, Bona and Winther showed that for any solution of (1.6) that is appropriately smooth up to the boundary, the following bounds hold:

$$
\sup _{0 \leq t \leq T}\|\eta(\cdot, t)\|_{H^{1}\left(R^{+}\right)} \leq \alpha_{1}\left(\|\phi\|_{H^{1}\left(R^{+}\right)}+\|h\|_{H^{2}(0, T)}\right)
$$

and

$$
\sup _{0 \leq t \leq T}\|\eta(\cdot, t)\|_{H^{s}\left(R^{+}\right)} \leq \alpha_{s}\left(\|\phi\|_{H^{s}\left(R^{+}\right)}+\|h\|_{H^{1+[s / 3]}(0, T)}\right),
$$

for $s=3 k$ or $3 k+1, k=1,2, \cdots$. The functions $\alpha_{j}: R^{+} \rightarrow R^{+}$are continuous and nondecreasing. It is worth pointing out that in the above estimates, more regularity of $h$ is required than that needed for the local well-posedness results indicated above and proved in Theorem 4.9. Moreover, missing from these results 
are direct bounds in $H^{2}\left(R^{+}\right), H^{5}\left(R^{+}\right), \cdots$, as well as in $H^{s}\left(R^{+}\right)$for non-integer $s$. An appraisal of the proof in Section 3 of [10] of these inequalities holds out little hope that such bounds will be forthcoming via direct energy estimates. Compared with the relative ease with which one obtains a priori estimates for the pure initialvalue problem, the IBVP (1.2) is more difficult because of the non-homogeneous boundary conditions and, at a more delicate level, the loss of regularity experienced when one takes the trace of a solution at $x=0$.

In this paper we will provide the following global a priori estimates for the IBVP (1.2): For any $T>0$,

$$
\sup _{0 \leq t \leq T}\|u(\cdot, t)\|_{H^{s}\left(R^{+}\right)} \leq \alpha_{s}\left(\|\phi\|_{H^{s}\left(R^{+}\right)}+\|h\|_{H^{\frac{7+3 s}{12}}(0, T)}\right)
$$

for $1 \leq s \leq 3$ and

$$
\sup _{0 \leq t \leq T}\|u(\cdot, t)\|_{H^{s}\left(R^{+}\right)} \leq \alpha_{s}\left(\|\phi\|_{H^{s}\left(R^{+}\right)}+\|h\|_{H^{\frac{s+1}{3}}(0, T)}\right)
$$

for $s \geq 3$. The various smoothing properties of (1.5) described earlier will play a central role in establishing these estimates. Another key tool is nonlinear interpolation theory as expounded in Tartar [57], and Bona and Scott [8].

Special arguments are also needed in discussing analyticity of the solution map $K_{I}$ associated to the IBVP (1.2) from the space $H^{s}\left(R^{+}\right) \times H^{s / 3}(0, T)$ to the space $C\left([0, T] ; H^{s}\left(R^{+}\right)\right)$. Because of the compatibility conditions that the initial data $\phi$ and the boundary data $h$ have to satisfy, the domain of the solution map $K_{I}$ is a linear subspace of $H^{s}\left(R^{+}\right) \times H^{s / 3}(0, T)$ only if $s \leq 7 / 2$. Thus the Taylor series expansion does not hold in the form depicted in (1.5) when $s>7 / 2$ and more subtle considerations are needed. In fact, it turns out to be convenient to generalize the setting to systems of $m$ equations that include the IBVP (1.2) as a special case. In this setting, an appropriate analyticity theory is formulated and proved, and the result then interpreted in the $\mathrm{KdV}$ setting to achieve an analyticity result for all $s>3 / 4$.

Finally, we point out that a linear problem related to (1.6), has been studied by Fokas and Pelloni [24], namely

$$
\left\{\begin{array}{l}
q_{t}+q_{x x x}=0, \quad x \geq 0, t>0, \\
q(x, 0)=q_{1}(x), \quad q(0, t)=q_{2}(t) .
\end{array}\right.
$$

They obtained the explicit solution

$$
q(x, t)=\frac{1}{2 \pi} \int_{-\infty}^{+\infty} e^{-i k x-i k^{3} t} \int_{0}^{\infty} e^{i k y} q_{1}(y) d y d k+\int_{L_{1}} e^{-i k x-i k^{3} t} \nu(k) d k,
$$

where the contour $L_{1}$ consists of the directed rays $\arg (k)=\frac{4}{3} \pi$ and $\arg (k)=\frac{5}{3} \pi$,

$$
\nu(k)=3 k^{2} \int_{0}^{\infty} e^{i k^{3} s} q_{2}(s) d s-\omega \hat{q_{1}}(\omega k)-\omega^{2} \hat{q}_{1}\left(\omega^{2} k\right), \quad \text { for } k \in L_{1},
$$

with $\omega=e^{-2 \pi i / 3}$ and

$$
\hat{q}_{1}(k)=\int_{0}^{\infty} e^{i k y} q_{1}(y) d y
$$


Of course, the term $q_{x}$ is missed from the equation in (1.21). To account for this, Fokas and Pelloni also considered the linear problem

$$
\begin{cases}q_{t}+q_{x x x}=0, & x \geq c t, t>0, \\ q(x, 0)=q_{1}(x), & x \geq 0, \\ q(c t, t)=q_{2}(t), & t \geq 0 .\end{cases}
$$

Under the Galilean transformation $x=\xi+c \tau, t=\tau$ mentioned in [24, (1.23) is equivalent to

$$
\begin{cases}q_{t}-c q_{x}+q_{x x x}=0, & x \geq 0, t>0, \\ q(x, 0)=q_{1}(x), & x \geq t, \\ q(0, t)=q_{2}(t), & t \geq 0 .\end{cases}
$$

The explicit solution

$$
q(x, t)=\frac{1}{2 \pi} \int_{-\infty}^{+\infty} e^{-i k x-i k^{3} t} \int_{0}^{\infty} e^{i k y} q_{1}(y) d y d k+\int_{L} e^{-i k x-i k^{3} t} \nu(k) d k,
$$

for the linear problem (1.23) is presented in [24], where the contour $L$ is the lower branch of hyperbola $3 k_{R}^{2}-k_{I}^{2}+c=0, k=k_{R}+i k_{I}$, in the complex $k$-plane and $\nu(k)$ is defined by $\nu(k)=G(k)+C(k)$ with

$$
G(k)=\left(3 k^{2}+c\right) \int_{0}^{\infty} e^{i\left(k^{3}+c k\right) s} q_{2}(s) d s
$$

and

$$
\begin{aligned}
C(k)= & \frac{3 k^{2}+2 c+3 k \bar{k}}{\left(3 k^{2}+2 c\right)\left(3 k^{2}+4\right)} \int_{0}^{\infty} e^{-i(k+\bar{k}) y} q_{1}(y) d y \\
& +\frac{3 k^{2}+2 c+3 k(k+\bar{k})}{\left(3 k^{2}+2 c\right)\left(3 k^{2}+4\right)} \int_{0}^{\infty} e^{-i \bar{k} y} q_{1}(y) d y
\end{aligned}
$$

Notice that one cannot take $c=-1$ in the latter formula, and hence this analysis does not provide a solution of the standard linear wave maker problem (1.6) as formulated above.

The plan of the present paper is as follows. Section 2 is devoted to the derivation of formal solution formulas for linear problems that lead to the results in (1.10)(1.11). This is somewhat tedious, but crucial in Section 3 to obtaining the linear estimates just described. Armed with these linear estimates, local well-posedness results are set down for auxiliary data in $H^{s}\left(R^{+}\right) \times H_{l o c}^{(s+1) / 3}$ provided only that $s>\frac{3}{4}$. At this stage of the development in Section 4, an interesting issue arises for larger values of $s$, which was already apparent in the work of Bona, Luo and Winther [6, 10, 11]. If $s$ is large enough, one needs more than the most obvious compatibility condition $\phi(0)=h(0)$ to infer existence of appropriately smooth solutions. Section 5 uses the local well-posedness theory, some a priori estimates, and nonlinear interpolation to attack global well-posedness in case $s \geq 1$. The last major section provides a theory pertaining to the analyticity of the solution mapping. 


\section{SOLUTION FORMULAS FOR LINEAR PROBLEMS}

In this section, explicit representation formulas are derived for solutions of initialboundary-value problems for the linear KdV equation.

Consideration is first directed to the homogeneous linear problem

$$
\begin{cases}u_{t}+u_{x}+u_{x x x}=0, & \text { for } x, t \geq 0, \\ u(x, 0)=\phi(x), & u(0, t)=0 .\end{cases}
$$

By semigroup theory, its solution may be obtained in the form

$$
u(t)=W_{c}(t) \phi,
$$

where the spatial variable is suppressed and $W_{c}(t)$ is the $C_{0}$-semigroup in the space $L^{2}\left(R^{+}\right)$generated by the operator

$$
A f=-f^{\prime \prime \prime}-f^{\prime}
$$

with the domain

$$
\mathcal{D}(A)=\left\{f \in H^{3}\left(R^{+}\right) \mid f(0)=0\right\} .
$$

By d'Alembert's formula, one may use the semigroup $W_{c}(t)$ to formally write the solution of the inhomogeneous linear problem

$$
\left\{\begin{array}{l}
u_{t}+u_{x}+u_{x x x}=f, \quad \text { for } x, t \geq 0, \\
u(x, 0)=0, \quad u(0, t)=0
\end{array}\right.
$$

in the form

$$
u(t)=\int_{0}^{t} W_{c}(t-\tau) f(\cdot, \tau) d \tau .
$$

The following proposition provides an explicit formula for $W_{c}(t) \phi$.

Proposition 2.1. For any $\phi \in L^{2}\left(R^{+}\right)$, define

$$
\begin{gathered}
U_{0}^{+}(t) \phi(x)=\frac{1}{2 \pi} \int_{1}^{\infty} e^{i \mu^{3} t-i \mu t} \int_{0}^{\infty} e^{i \mu(x-\xi)} \phi(\xi) d \xi d \mu \\
U_{1}^{+}(t) \phi(x)=-\frac{1}{2 \pi} \int_{1}^{\infty} e^{i \mu^{3} t-i \mu t} e^{-\left(\frac{i \mu+\sqrt{3 \mu^{2}-4}}{2}\right) x} \int_{0}^{\infty} e^{-i \mu \xi} \phi(\xi) d \xi d \mu
\end{gathered}
$$

and

$$
U_{2}^{+}(t) \phi(x)=\frac{1}{2 \pi i} \int_{0}^{\infty} e^{-\mu^{3} t-\mu t} e^{-\left(\frac{\mu-i \sqrt{3 \mu^{2}+4}}{2}\right) x} \int_{0}^{\infty} e^{-\mu \xi} \phi(\xi) d \xi d \mu .
$$

Then it follows that

$$
W_{c}(t) \phi(x)=\sum_{j=0}^{2}\left(U_{j}^{+}(t) \phi(x)+\overline{U_{j}^{+}(t) \phi(x)}\right) .
$$

Proof. Using the Laplace transform as described earlier, there obtains the formula

$$
u(x, t)=\frac{1}{2 \pi i} \int_{r-i \infty}^{r+i \infty} e^{\lambda t} R(\lambda, A) \phi(x) d \lambda
$$


where $r>0$ is a given constant and $R(\lambda, A)$ is the resolvent operator of $A$. If the function $v$ is defined by $v=R(\lambda, A) \phi$, then $v$ is a solution of

$$
\left\{\begin{array}{l}
\lambda v(x)+v^{\prime}(x)+v^{\prime \prime \prime}(x)=\phi(x), \quad 0 \leq x<+\infty \\
v(0)=0, \quad v(x), v^{\prime}(x) \rightarrow 0 \text { as } x \rightarrow+\infty
\end{array}\right.
$$

In consequence, the function $v$ has the representation

$$
v(x)=R(\lambda, A) \phi(x)=\int_{0}^{\infty} G(x, s, \lambda) \phi(s) d s,
$$

where $G=G(x, s, \lambda)$ is the associated Green's function for (2.4). If we let $\gamma_{1}, \gamma_{2}, \gamma_{3}$ be the three roots of the characteristic equation

$$
\lambda+\gamma+\gamma^{3}=0, \quad \text { for } \operatorname{Re} \lambda>0,
$$

ordered so that

$$
\operatorname{Re} \gamma_{1}<0, \quad \operatorname{Re} \gamma_{2}>0, \quad \operatorname{Re} \gamma_{3}>0
$$

then $G$ is given explicitly by

$$
\begin{aligned}
G(x, s, \lambda)= & \frac{1}{\Delta(\lambda)}\left\{\left(\gamma_{3}-\gamma_{1}\right) e^{\gamma_{1} x-\gamma_{2} s}+\left(\gamma_{1}-\gamma_{2}\right) e^{\gamma_{1} x-\gamma_{3} s}\right. \\
& +Y(x, s)\left(\gamma_{2}-\gamma_{3}\right) e^{\gamma_{1}(x-s)} \\
& \left.+(1-Y(x, s))\left(\left(\gamma_{1}-\gamma_{3}\right) e^{\gamma_{2}(x-s)}+\left(\gamma_{2}-\gamma_{1}\right) e^{\gamma_{3}(x-s)}\right)\right\}
\end{aligned}
$$

where

$$
\Delta(\lambda)=\left(\gamma_{1}-\gamma_{2}\right)\left(\gamma_{1}-\gamma_{3}\right)\left(\gamma_{2}-\gamma_{3}\right)
$$

and

$$
Y(x, s)= \begin{cases}1 & \text { if } 0 \leq s \leq x \\ 0 & \text { otherwise }\end{cases}
$$

Combining these formulas gives the representation

$$
W_{c}(t) \phi(x)=\frac{1}{2 \pi i} \int_{r-i \infty}^{r+i \infty} e^{\lambda t} \int_{0}^{\infty} G(x, s, \lambda) \phi(s) d s d \lambda,
$$

valid for any fixed $r>0$. Note that, for fixed values of $x$ and $s, G(x, s, \lambda)$ is analytic in the right-half plane $\operatorname{Re} \lambda>0$ and continuous for $\operatorname{Re} \lambda \geq 0$ except at

$$
\lambda=\nu_{ \pm} \equiv \pm \frac{2 i}{3 \sqrt{3}} \text {. }
$$

But, as $\lambda \rightarrow \nu_{ \pm}$, a little analysis shows that

$$
G(x, s, \lambda) \sim O\left(\left|\lambda-\nu_{ \pm}\right|^{-1 / 2}\right)
$$

uniformly for $x, s \geq 0$. As this singularity is integrable, we may let $r \rightarrow 0$ in (2.6), thereby obtaining

$$
\begin{aligned}
W_{c}(t) \phi(x) & =\frac{1}{2 \pi i} \int_{-i \infty}^{i \infty} e^{\lambda t} \int_{0}^{\infty} G(x, s, \lambda) \phi(s) d s d \lambda \\
& \equiv I+I I
\end{aligned}
$$


with

$$
I=\frac{1}{2 \pi i} \int_{0}^{i \infty} e^{\lambda t} \int_{0}^{\infty} G(x, s, \lambda) \phi(s) d s d \lambda
$$

and

$$
I I=\frac{1}{2 \pi i} \int_{-i \infty}^{0} e^{\lambda t} \int_{0}^{\infty} G(x, s, \lambda) \phi(s) d s d \lambda .
$$

Introduce the notation

$$
\begin{gathered}
A(x, \lambda)=A_{1}(x, \lambda)+A_{2}(x, \lambda), \\
B(x, \lambda)=\int_{0}^{x}\left(\gamma_{2}-\gamma_{3}\right) e^{\gamma_{1}(x-\xi)} \phi(\xi) d \xi
\end{gathered}
$$

and

$$
C(x, \lambda)=C_{1}(x, \lambda)+C_{2}(x, \lambda)
$$

with

$$
\begin{aligned}
& A_{1}(x, \lambda)=\int_{0}^{\infty}\left(\gamma_{3}-\gamma_{1}\right) e^{\gamma_{1} x-\gamma_{2} \xi} \phi(\xi) d \xi \\
& A_{2}(x, \lambda)=\int_{0}^{\infty}\left(\gamma_{1}-\gamma_{2}\right) e^{\gamma_{1} x-\gamma_{3} \xi} \phi(\xi) d \xi \\
& C_{1}(x, \lambda)=\int_{x}^{\infty}\left(\gamma_{1}-\gamma_{3}\right) e^{\gamma_{2}(x-\xi)} \phi(\xi) d \xi
\end{aligned}
$$

and

$$
C_{2}(x, \lambda)=\int_{x}^{\infty}\left(\gamma_{2}-\gamma_{1}\right) e^{\gamma_{3}(x-\xi)} \phi(\xi) d \xi .
$$

Then the quantities $I$ and $I I$ may be written in the form

$$
I=\frac{1}{2 \pi i} \int_{0}^{\infty i} \frac{e^{\lambda t}}{\Delta(\lambda)}[A(x, \lambda)+B(x, \lambda)+C(x, \lambda)] d \lambda
$$

and

$$
I I=\frac{1}{2 \pi i} \int_{-\infty i}^{0} \frac{e^{\lambda t}}{\Delta(\lambda)}[A(x, \lambda)+B(x, \lambda)+C(x, \lambda)] d \lambda .
$$

Consider first the integral $I$. It is convenient to make the change of variables

$$
\lambda=i \mu^{3}-i \mu, \quad \text { with } \quad 1 \leq \mu<+\infty,
$$

in the equation

$$
\gamma^{3}+\gamma+\lambda=0
$$

In terms of $\mu$, the three solutions of the characteristic equation 2.5) are

$$
\gamma_{1}^{+}=\frac{-\sqrt{3 \mu^{2}-4}-i \mu}{2}, \quad \gamma_{2}^{+}=\frac{\sqrt{3 \mu^{2}-4}-i \mu}{2}, \quad \gamma_{3}^{+}=i \mu .
$$

Notice that

$$
\gamma_{1}^{+}(1)=-i \quad \text { and } \quad \gamma_{2}^{+}(1)=0
$$


In terms of the variable $\mu$, the integral $I$ may be rewritten as

$$
I=\frac{1}{2 \pi i} \int_{1}^{\infty} \frac{e^{i \mu^{3} t-i \mu t}}{\Delta^{+}(\mu)} i\left(3 \mu^{2}-1\right)\left(A^{+}(x, \mu)+B^{+}(x, \mu)+C^{+}(x, \mu)\right) d \mu,
$$

where $\Delta^{+}(\mu), A^{+}(x, \mu), B^{+}(x, \mu)$ and $C^{+}(x, \mu)$ are obtained from $\Delta(x, \lambda), A(x, \lambda)$, $B(x, \lambda)$ and $C(x, \lambda)$, respectively, by replacing $\lambda$ with $i \mu^{3}-i \mu$ and $\gamma_{1}, \gamma_{2}$ and $\gamma_{3}$ by $\gamma_{1}^{+}, \gamma_{2}^{+}$and $\gamma_{3}^{+}$, respectively.

Similarly, in the analysis of the integral $I I$, make the change of variables

$$
\lambda=\overline{i \mu^{3}-i \mu}=-i \mu^{3}+i \mu, \quad \text { where } \quad \mu \geq 1,
$$

in the equation

$$
\gamma^{3}+\gamma+\lambda=0
$$

The three roots of this characteristic equation are given by

$$
\gamma_{1}^{-}(\mu)=\overline{\gamma_{1}^{+}(\mu)}, \quad \gamma_{2}^{-}(\mu)=\overline{\gamma_{2}^{+}(\mu)}, \quad \gamma_{3}^{-}(\mu)=\overline{\gamma_{3}^{+}(\mu)},
$$

in this case. Thus, it transpires that

$$
I I=\frac{1}{2 \pi i} \int_{+\infty}^{1} \frac{e^{-i \mu^{3} t+i \mu t}}{\Delta^{-}(\mu)} i\left(1-3 \mu^{2}\right)\left(A^{-}(x, \mu)+B^{-}(x, \mu)+C^{-}(x, \mu)\right) d \mu,
$$

where

$$
\begin{aligned}
\Delta^{-}(\mu) & =\overline{\Delta^{+}(\mu)}, \quad A^{-}(x, \mu)=\overline{A^{+}(x, \mu)}, \\
B^{-}(x, \mu) & =\overline{B^{+}(x, \mu)}, \quad C^{-}(x, \mu)=\overline{C^{+}(x, \mu)} .
\end{aligned}
$$

It is now proposed that

$$
\begin{aligned}
\overline{U_{0}^{+}(t) \phi(x)} & =\frac{1}{2 \pi i} \int_{1}^{\infty} \frac{e^{i \mu^{3} t-i \mu t}}{\Delta^{+}(\mu)} i\left(3 \mu^{2}-1\right) B^{+}(x, \mu) d \mu \\
& +\frac{1}{2 \pi i} \int_{+\infty}^{1} \frac{e^{-i \mu^{3} t+i \mu t}}{\Delta^{-}(\mu)} i\left(1-3 \mu^{2}\right) C_{2}^{-}(x, \mu) d \mu,
\end{aligned}
$$

from which it transpires that

$$
\begin{aligned}
U_{0}^{+}(t) \phi(x) & =\frac{1}{2 \pi i} \int_{+\infty}^{1} \frac{e^{-i \mu^{3} t+i \mu t}}{\Delta^{-}(\mu)} i\left(1-3 \mu^{2}\right) B^{-}(x, \mu) d \mu \\
& +\frac{1}{2 \pi i} \int_{1}^{\infty} \frac{e^{i \mu^{3} t-i \mu t}}{\Delta^{+}(\mu)} i\left(3 \mu^{2}-1\right) C_{2}^{+}(x, \mu) d \mu
\end{aligned}
$$

To see the validity of (2.7), let

$$
\Gamma_{3}^{+}=\left\{z: z=\gamma_{3}^{+}(\mu), 1 \leq \mu<+\infty\right\}
$$

and

$$
\Gamma_{1}^{-}=\left\{z: z=\gamma_{1}^{-}(\mu), 1 \leq \mu<+\infty\right\} .
$$

Both $\Gamma_{1}^{-}$and $\Gamma_{3}^{+}$are oriented curves in the complex $z$-plane starting at the same point $z=i$. Let $\Omega_{1}$ denote the region enclosed by $\Gamma_{1}^{-}$and $\Gamma_{3}^{+}$that includes a 
neighborhood of the imaginary axis from $i$ to $i \infty$ with negative real part. By a simple change of variables,

$$
\frac{1}{2 \pi i} \int_{1}^{\infty} \frac{e^{i \mu^{3} t-i \mu t}}{\Delta^{+}(\mu)} i\left(3 \mu^{2}-1\right) B^{+}(x, \mu) d \mu=\frac{1}{2 \pi i} \int_{\Gamma_{3}^{+}} F_{1}^{+}(x, t, z) d z
$$

with

$$
F_{1}^{+}(x, t, z)=e^{-\left(z^{3}+z\right) t} \tilde{B}^{+}(x, z)\left(-3 z^{2}-1\right) / \tilde{\Delta}^{+}(z)
$$

where

$$
\begin{gathered}
\tilde{B}^{+}(x, z)=\int_{0}^{x}\left(\tilde{\gamma}_{2}^{+}(z)-\tilde{\gamma}_{3}^{+}(z)\right) e^{\tilde{\gamma}_{1}^{+}(z)(x-\xi)} \phi(\xi) d \xi, \\
\tilde{\Delta}^{+}(z)=\left(\tilde{\gamma}_{1}^{+}(z)-\tilde{\gamma}_{2}^{+}(z)\right)\left(\tilde{\gamma}_{1}^{+}(z)-\tilde{\gamma}_{3}^{+}(z)\right)\left(\tilde{\gamma}_{2}^{+}(z)-\tilde{\gamma}_{3}^{+}(z)\right),
\end{gathered}
$$

and

$$
\tilde{\gamma}_{1}^{+}(z)=\frac{-\sqrt{-3 z^{2}-4}-z}{2}, \quad \tilde{\gamma}_{2}^{+}(z)=\frac{\sqrt{-3 z^{2}-4}-z}{2}, \quad \tilde{\gamma}_{3}^{+}=z .
$$

The function $F_{1}^{+}(x, t, z)$ is analytic in the region $\Omega_{1}$ and is continuous on the closure of $\Omega_{1}$. In addition, $F_{1}^{+}(x, t, z)$ tends to zero as $z \rightarrow \infty$ in $\Omega_{1}$, uniformly in $x$ and $t$. Hence, we are allowed to change the contour on the basis of Cauchy's Theorem and thereby determine that

$$
\frac{1}{2 \pi i} \int_{\Gamma_{3}^{+}} F_{1}^{+}(x, t, z) d z=\frac{1}{2 \pi i} \int_{\Gamma_{1}^{-}} F_{1}^{+}(x, t, z) d z .
$$

But, on $\Gamma_{1}^{-}$, we see that

$$
\begin{gathered}
z^{3}+z=\left(\gamma_{1}^{-}(\mu)\right)^{3}+\gamma_{1}^{-}(\mu)=i \mu^{3}-i \mu, \\
\left(3 z^{2}+1\right) d z=d\left(z^{3}+z\right)=i\left(3 \mu^{2}-1\right) d \mu, \\
\tilde{\gamma}_{1}^{+}(z)=\gamma_{3}^{-}(\mu), \tilde{\gamma}_{2}^{+}(z)=\gamma_{2}^{-}(\mu), \tilde{\gamma}_{3}^{+}(z)=\gamma_{1}^{-}(\mu), \\
\tilde{\Delta}^{+}(z)=\left(\gamma_{3}^{-}(\mu)-\gamma_{2}^{-}(\mu)\right)\left(\gamma_{3}^{-}(\mu)-\gamma_{1}^{-}(\mu)\right)\left(\gamma_{2}^{-}(\mu)-\gamma_{1}^{-}(\mu)\right) \\
=\left(1-3 \mu^{2}\right) \overline{\sqrt{3 \mu^{2}-4}},
\end{gathered}
$$

and

$$
\tilde{B}^{+}(x, z)=\int_{0}^{x} \overline{\sqrt{3 \mu^{2}-4}} e^{-i \mu(x-\xi)} \phi(\xi) d \xi .
$$

In consequence, it follows that

$$
\frac{1}{2 \pi i} \int_{\Gamma_{3}^{+}} F_{1}^{+}(x, t, z) d z=\frac{1}{2 \pi} \int_{1}^{\infty} e^{-i \mu^{3} t+i \mu t} \int_{0}^{x} e^{-i \mu(x-\xi)} \phi(\xi) d \xi d \mu .
$$

A direct computation reveals that

$$
\begin{aligned}
& \frac{1}{2 \pi i} \int_{+\infty}^{1} \frac{e^{-i \mu^{3} t+i \mu t}}{\Delta^{-}(\mu)} i\left(1-3 \mu^{2}\right) C_{2}^{-}(x, \mu) d \mu \\
= & \frac{1}{2 \pi} \int_{1}^{\infty} e^{-i \mu^{3} t+i \mu t} \int_{x}^{+\infty} e^{-i \mu(x-\xi)} \phi(\xi) d \xi d \mu,
\end{aligned}
$$

whence (2.7) holds. 
Next, it is established that

$$
\begin{gathered}
\frac{1}{2 \pi i} \int_{1}^{\infty} \frac{e^{i \mu^{3} t-i \mu t}}{\Delta^{+}(\mu)} i\left(3 \mu^{2}-1\right) C_{1}^{+}(x, \mu) d \mu \\
+\frac{1}{2 \pi i} \int_{+\infty}^{1} \frac{e^{-i \mu^{3} t+i \mu t}}{\Delta^{-}(\mu)} i\left(1-3 \mu^{2}\right) C_{1}^{-}(x, \mu) d \mu=0 .
\end{gathered}
$$

To prove this formula, let $\lambda=-\mu^{3}-\mu$ in the equation

$$
\gamma^{3}+\gamma+\lambda=0
$$

where $\mu \geq 0$. Write the three solutions of the characteristic equation as

$$
\gamma_{1}^{*}(\mu)=\frac{-\mu+i \sqrt{4+3 \mu^{2}}}{2}, \quad \gamma_{2}^{*}(\mu)=\frac{-\mu-i \sqrt{4+3 \mu^{2}}}{2}, \quad \gamma_{3}^{*}(\mu)=\mu .
$$

We have that $\gamma_{1}^{*}(0)=i=\gamma_{3}^{+}(1)$. Let

$$
\Gamma_{1}^{*}=\left\{z=\gamma_{1}^{*}(\mu): \quad 0 \leq \mu<+\infty\right\}
$$

and let $\Omega_{1}^{*}$ be the open region enclosed by $\Gamma_{1}^{*}$ and $\Gamma_{3}^{+}$. We may write

$$
\frac{1}{2 \pi i} \int_{1}^{\infty} \frac{e^{i \mu^{3} t-i \mu t}}{\Delta^{+}(\mu)} i\left(3 \mu^{2}-1\right) C_{1}^{+}(x, \mu) d \mu=\frac{1}{2 \pi i} \int_{\Gamma_{3}^{+}} F_{2}^{+}(x, t, z) d z
$$

with

$$
F_{2}^{+}(x, t, z)=e^{-\left(z^{3}+z\right) t} \frac{-\left(3 z^{2}+1\right)}{\tilde{\Delta}^{+}(z)} \int_{x}^{\infty}\left(\tilde{\gamma}_{1}^{+}(z)-\tilde{\gamma}_{3}^{+}(z)\right) e^{\tilde{\gamma}_{2}^{+}(z)(x-\xi)} \phi(\xi) d \xi .
$$

Since $F_{2}^{+}(x, t, z)$ is analytic in $\Omega_{1}^{*}$ and is continuous on $\overline{\Omega_{1}^{*}}$ and tends to zero as $z \rightarrow \infty$ in $\Omega_{1}^{*}$, we can change the contour of integration and conclude

$$
\frac{1}{2 \pi i} \int_{\Gamma_{3}^{+}} F_{2}^{+}(x, t, z) d z=\frac{1}{2 \pi i} \int_{\Gamma_{1}^{*}} F_{2}^{+}(x, t, z) d z .
$$

On $\Gamma_{1}^{*}$, for $0 \leq \mu<+\infty$,

$$
\begin{gathered}
z^{3}+z=\mu^{3}+\mu, \quad\left(3 z^{2}+1\right) d z=\left(3 \mu^{2}+1\right) d \mu, \\
\tilde{\gamma}_{1}^{+}(z)=\gamma_{2}^{*}(\mu), \tilde{\gamma}_{2}^{+}(z)=\gamma_{3}^{*}(\mu), \tilde{\gamma}_{3}^{+}(z)=\gamma_{1}^{*}(\mu), \\
\tilde{\Delta}^{+}(z)=\left(\gamma_{2}^{*}(\mu)-\gamma_{3}^{*}(\mu)\right)\left(\gamma_{2}^{*}(\mu)-\gamma_{1}^{*}(\mu)\right)\left(\gamma_{3}^{*}(\mu)-\gamma_{1}^{*}(\mu)\right) \\
=i\left(1+3 \mu^{2}\right) \sqrt{3 \mu^{2}+4},
\end{gathered}
$$

and

$$
\tilde{\gamma}_{1}^{+}(z)-\tilde{\gamma}_{3}^{+}(z)=-i \sqrt{3 \mu^{2}+4} \text {. }
$$

Consequently,

$$
\frac{1}{2 \pi i} \int_{\Gamma_{3}^{+}} F_{2}^{+}(x, t, z) d z=\frac{1}{2 \pi i} \int_{0}^{+\infty} e^{-\left(\mu^{3}+\mu\right) t} \int_{x}^{\infty} e^{\mu(x-\xi)} \phi(\xi) d \xi d \mu .
$$


In addition,

$$
\begin{aligned}
\int_{+\infty}^{1} & \frac{e^{-i \mu^{3} t+i \mu t}}{\Delta^{-}(\mu)} i\left(1-3 \mu^{2}\right) C_{1}^{-}(x, \mu) d \mu=-\overline{\int_{1}^{\infty} \frac{e^{i \mu^{3} t-i \mu t}}{\Delta^{+}(\mu)} i\left(3 \mu^{2}-1\right) C_{1}^{+}(x, \mu) d \mu} \\
= & -\int_{0}^{+\infty} e^{-\left(\mu^{3}+\mu\right) t} \int_{x}^{\infty} e^{\mu(x-\xi)} \phi(\xi) d \xi d \mu .
\end{aligned}
$$

The proposition in view is thus established.

Similarly, by appropriate changes of the contour of integration, it is inferred that

$$
\frac{1}{2 \pi i} \int_{1}^{\infty} \frac{e^{i \mu^{3} t-i \mu t}}{\Delta^{+}(\mu)} i\left(3 \mu^{2}-1\right) A_{1}^{+}(x, \mu) d \mu=-\overline{U_{2}^{+}(t) \phi(x)}
$$

and

$$
\frac{1}{2 \pi i} \int_{+\infty}^{1} \frac{e^{-i \mu^{3} t+i \mu t}}{\Delta^{-}(\mu)} i\left(1-3 \mu^{2}\right) A_{1}^{-}(x, \mu) d \mu=U_{2}^{+}(t) \phi(x) .
$$

Finally, a direct computation shows that

$$
\frac{1}{2 \pi i} \int_{1}^{\infty} \frac{e^{i \mu^{3} t-i \mu t}}{\Delta^{+}(\mu)} i\left(3 \mu^{2}-1\right) A_{2}^{+}(x, \mu) d \mu=U_{1}^{+}(t) \phi(x)
$$

and

$$
\frac{1}{2 \pi i} \int_{+\infty}^{1} \frac{e^{-i \mu^{3} t+i \mu t}}{\Delta^{-}(\mu)} i\left(1-3 \mu^{2}\right) A_{2}^{-}(x, \mu) d \mu=U_{1}^{-}(t) \phi(x) .
$$

The proof is complete.

Next, consideration is given to the non-homogeneous boundary-value problem

$$
\left\{\begin{array}{l}
u_{t}+u_{x}+u_{x x x}=0, \quad \text { for } x, t \geq 0, \\
u(x, 0)=0, \quad u(0, t)=h(t) .
\end{array}\right.
$$

Proposition 2.2. The solution of (2.9) may be written as

$$
u(x, t)=\left[W_{b}(t) h\right](x)=\left[U_{b}(t) h\right](x)+\overline{\left[U_{b}(t) h\right](x)}
$$

where, for $x, t \geq 0$,

$$
\left[U_{b}(t) h\right](x)=\frac{1}{2 \pi} \int_{1}^{\infty} e^{i \mu^{3} t-i \mu t} e^{-\left(\frac{\sqrt{3 \mu^{2}-4}+i \mu}{2}\right) x}\left(3 \mu^{2}-1\right) \int_{0}^{\infty} e^{-\left(\mu^{3} i-i \mu\right) \xi} h(\xi) d \xi d \mu .
$$

Proof. Let $\tilde{u}$ and $\tilde{h}$ denote the Laplace transform of $u$ and $h$ with respect to $t$, respectively. By applying the Laplace transform to both sides of the equation in (2.9), one obtains

$$
\lambda \tilde{u}(x, \lambda)+\tilde{u}_{x}(x, \lambda)+\tilde{u}_{x x x}(x, \lambda)=0, \quad \tilde{u}(0, \lambda)=\tilde{h}(\lambda) .
$$

As both $\tilde{u}(x, \lambda)$ and $\tilde{u}_{x}(x, \lambda)$ tend to zero as $x \rightarrow \infty$, it is concluded that for any $\lambda$ with $\operatorname{Re} \lambda>0$,

$$
\tilde{u}(x, \lambda)=\tilde{h}(\lambda) e^{r_{1}(\lambda) x}
$$

where $r_{1}(\lambda)$ is the unique solution of

$$
\lambda+r^{3}+r=0
$$


satisfying $\operatorname{Re} r_{1}(\lambda)<0$. Thus, for any fixed $\gamma>0$, one has the representation

$$
u(x, t)=\frac{1}{2 \pi i} \int_{-i \infty+\gamma}^{i \infty+\gamma} e^{\lambda t} \tilde{h}(\lambda) e^{r_{1}(\lambda) x} d \lambda
$$

Arguing as in Proposition 2.1 and using the fact that the right-hand side of this relation is continuous in $\gamma$ up to $\gamma=0$, there obtains

$$
u(x, t)=\frac{1}{2 \pi i} \int_{0}^{i \infty} e^{\lambda t} \tilde{h}(\lambda) e^{r_{1}(\lambda) x} d \lambda+\frac{1}{2 \pi i} \int_{-i \infty}^{0} e^{\lambda t} \tilde{h}(\lambda) e^{r_{1}(\lambda) x} d \lambda .
$$

On the positive imaginary axis, take $\lambda$ in the form $\lambda=i \mu^{3}-i \mu$ for a unique $\mu$ with $1 \leq \mu<+\infty$. In terms of $\mu$, the quantity $r_{1}(\lambda)$ has the value

$$
r_{1}(\lambda)=-\frac{\sqrt{3 \mu^{2}-4}+i \mu}{2}
$$

as before. By direct computation, it follows that for $x, t \geq 0$,

$$
\frac{1}{2 \pi i} \int_{0}^{i \infty} e^{\lambda t} \tilde{h}(\lambda) e^{r_{1}(\lambda) x} d \lambda=\left[U_{b}(t) h\right](x) .
$$

Similar reasoning shows that

$$
\frac{1}{2 \pi i} \int_{-i \infty}^{0} e^{\lambda t} \tilde{h}(\lambda) e^{r_{1}(\lambda) x} d \lambda=\overline{\left[U_{b}(t) h\right](x)}
$$

thus completing the proof.

Finally, attention is turned to the inhomogeneous initial-boundary-value problem

$$
\begin{cases}u_{t}+u_{x}+u_{x x x}=f, & \text { for } x, t \geq 0, \\ u(x, 0)=\phi(x), & u(0, t)=h(t),\end{cases}
$$

where $\phi$ and $h$ are assumed to satisfy the compatibility condition $h(0)=\phi(0)$. Let $u(x, t)=z(x, t)+e^{-x-t} h(0)$. It is easy to see that if $u$ solves (2.11), then $z(x, t)$ solves

$$
\left\{\begin{array}{l}
z_{t}+z_{x}+z_{x x x}=f+2 e^{-x-t} h(0), \quad \text { for } x, t \geq 0 \\
z(x, 0)=\phi(x)-e^{-x} \phi(0), \quad z(0, t)=h(t)-e^{-t} h(0)
\end{array}\right.
$$

Decompose $z$ in the form $z=w+v+y$ with

$$
\begin{aligned}
& \left\{\begin{array}{l}
w_{t}+w_{x}+w_{x x x}=f+2 e^{-x-t} h(0), \quad \text { for } x, t \geq 0, \\
w(x, 0)=0, \quad w(0, t)=0,
\end{array}\right. \\
& \left\{\begin{array}{l}
v_{t}+v_{x}+v_{x x x}=0, \quad \text { for } x, t \geq 0, \\
v(x, 0)=\phi(x)-e^{-x} \phi(0),
\end{array}, \quad v(0, t)=0,\right.
\end{aligned}
$$

and

$$
\left\{\begin{array}{l}
y_{t}+y_{x}+y_{x x x}=0, \quad \text { for } x, t \geq 0 \\
y(x, 0)=0, \quad y(0, t)=h(t)-e^{-t} h(0)
\end{array}\right.
$$

The following representation for the solution of (2.11) emerges from this decomposition together with the results of Lemmas 2.1 and 2.2 and Duhamel's principle. 
Proposition 2.3. The solution $u(x, t)$ of (2.11) is

$$
\begin{gathered}
u(x, t)=W_{c}(t)\left(\phi(x)-e^{-x} \phi(0)\right)+\int_{0}^{t} W_{c}(t-\tau)\left(f(x, \tau)+2 e^{-x-\tau} h(0)\right) d \tau \\
+\left[W_{b}(t)\left(h(t)-e^{-t} h(0)\right)\right](x)+e^{-x-t} h(0) .
\end{gathered}
$$

\section{LineAR EStimates}

In this section, estimates for the semigroups $W_{c}(t)$ and $W_{b}(t)$ are obtained. These estimates, analogous to those obtained by Kenig et al. [41, 42, for the linear $\mathrm{KdV}$ equation posed on the whole line $R$, reveal various smoothing properties of the semigroups $W_{c}(t)$ and $W_{b}(t)$ and will play an important role in establishing well-posedness results for the nonlinear problem in Sections 4 and 5 .

We start by laying out notation for the fractional-order Sobolev classes defined on $R^{+}$. For $s \geq 0$, write $s=m+\theta$ where $0 \leq \theta<1$ and $m$ is a non-negative integer. Thus $m=[s]$, the greatest integer in $s$. For $f \in C^{\infty}\left(R^{+}\right) \cap H^{m}\left(R^{+}\right)$, define a new function $J_{x}^{s} f$ by

$$
J_{x}^{s} f(x)= \begin{cases}\left|f^{(m)}(x)\right| & \text { if } \theta=0, \\ \left(\int_{0}^{\infty} \tau^{-(2 \theta+1)}\left|f^{(m)}(x+\tau)-f^{(m)}(x)\right|^{2} d \tau\right)^{1 / 2}, & \text { if } \theta>0,\end{cases}
$$

for any $x \in R^{+}$. Because $f^{(m)}$ is smooth and an $L^{2}\left(R^{+}\right)$-function and $\theta<1, J_{x}^{s} f(x)$ is finite for all $x$. The quantity

$$
\|f\|_{H^{s}\left(R^{+}\right)}^{2}=\|f\|_{L^{2}\left(R^{+}\right)}^{2}+\left\|J_{x}^{s} f\right\|_{L^{2}\left(R^{+}\right)}^{2}
$$

defines a norm on $C^{\infty}\left(R^{+}\right) \cap H^{m}\left(R^{+}\right)$and the completion of this space in the norm (3.1) is denoted by $H^{s}\left(R^{+}\right)$. The space $H_{0}^{s}\left(R^{+}\right)$is the completion of $C_{0}^{\infty}\left(R^{+}\right)$in the norm defined in (3.1). Clearly $H_{0}^{s}\left(R^{+}\right)$is a closed linear subspace of $H^{s}\left(R^{+}\right)$ and

$$
H_{0}^{s}\left(R^{+}\right)=H^{s}\left(R^{+}\right)
$$

if $0 \leq s \leq 1 / 2$. A good reference for this material is Lions and Magenes [47.

Next we present two technical lemmas that will find frequent use in this section. They play the same role in dealing with the half-line problem as does Plancherel's Theorem for the $\mathrm{KdV}$ equation posed on the entire real line.

Lemma 3.1. For any $f \in L^{2}(a,+\infty)$, let $K f$ be the function defined by

$$
K f(x)=\int_{a}^{+\infty} e^{\gamma(\mu) x} f(\mu) d \mu,
$$

where $a \in R$ and $\gamma(\mu)$ is a continuous complex-valued function defined on $(a, \infty)$ satisfying the following three conditions:

(i) $\operatorname{Re} \gamma(\mu)<0$, for $\mu>a$;

(ii) there exist $\delta>0$ and $b>0$ such that

$$
\sup _{a<\mu<a+\delta} \frac{|\operatorname{Re} \gamma(\mu)|}{\mu-a} \geq b ;
$$


(iii) there exists a complex number $\alpha+i \beta$ with $\alpha<0$ such that

$$
\lim _{\mu \rightarrow+\infty} \frac{\gamma(\mu)}{\mu}=\alpha+i \beta .
$$

Then there exists a constant $C$ such that for all $f \in L^{2}(a, \infty)$,

$$
\|K f\|_{L^{2}\left(R^{+}\right)} \leq C\|f\|_{L^{2}(a, \infty)} .
$$

Proof. By a translation of the coordinate system, we may assume $a=0$. In this frame of reference, our assumptions imply that there is a positive constant $d$ such that

$$
-\operatorname{Re}(\gamma(r)) \geq d r
$$

for all $r \geq 0$. Next, observe that

$$
\begin{aligned}
\|K f\|_{L^{2}\left(R^{+}\right)}^{2} & \leq \int_{0}^{+\infty}\left\{\int_{0}^{+\infty} e^{\operatorname{Re}(\gamma(s) x)}|f(s)| d s \int_{0}^{+\infty} e^{\operatorname{Re}(\gamma(t) x)}|f(t)| d t\right\} d x \\
& =\int_{0}^{+\infty} \int_{0}^{+\infty} \int_{0}^{+\infty} e^{\operatorname{Re}(\gamma(s)+\gamma(t)) x}|f(s) \| f(t)| d x d s d t \\
& =\int_{0}^{+\infty} \int_{0}^{+\infty} \frac{|f(s) f(t)|}{|\operatorname{Re}(\gamma(s)+\gamma(t))|} d s d t \\
& \leq\left\|\int_{0}^{+\infty} \frac{|f(s)| d s}{|\operatorname{Re}(\gamma(s)+\gamma(t))|}\right\|_{L_{t}^{2}\left(R^{+}\right)}\|f\|_{L^{2}\left(R^{+}\right)}
\end{aligned}
$$

by the Cauchy-Schwarz inequality. Changing variables and using the integral version of Minkowski's inequality yields

$$
\begin{aligned}
& \left\|\int_{0}^{+\infty} \frac{|f(s)| d s}{|\operatorname{Re}(\gamma(s)+\gamma(t))|}\right\|_{L_{t}^{2}\left(R^{+}\right)}=\left\|\int_{0}^{+\infty} \frac{|f(\mu t) t| d \mu}{|\operatorname{Re}(\gamma(\mu t)+\gamma(t))|}\right\|_{L_{t}^{2}\left(R^{+}\right)} \\
& \quad \leq \int_{0}^{+\infty}\left\|\frac{f(\mu t) t}{\operatorname{Re}(\gamma(\mu t)+\gamma(t))}\right\|_{L_{t}^{2}\left(R^{+}\right)} d \mu \\
& \quad \leq C \int_{0}^{\infty} \frac{1}{\sqrt{\mu}(1+\mu)} d \mu\|f\|_{L^{2}\left(R^{+}\right)} \\
& \quad \leq C\|f\|_{L^{2}\left(R^{+}\right)}
\end{aligned}
$$

since

$$
\|f(\mu t)\|_{L_{t}^{2}\left(R^{+}\right)}=\mu^{-1 / 2}\|f\|_{L^{2}\left(R^{+}\right)}
$$

and, because of (3.2),

$$
\frac{t}{|\operatorname{Re}(\gamma(\mu t)+\gamma(t))|} \leq \frac{1}{d(\mu+1)}
$$

for any $t \in(0,+\infty)$. The proof is complete.

Lemma 3.2. Let $a>0$ be given. For any $f \in L^{2}(0, a)$, let $G f$ be the function defined by

$$
G f(x)=\int_{0}^{a} e^{i \xi(\mu) x} f(\mu) d \mu
$$


where $\xi(\mu)$ is a continuous real-valued function defined on the interval $[0, a]$ which is $C^{1}$ on the open interval $(0, a)$ and satisfies the two conditions:

(i) $\xi^{\prime}(\mu) \neq 0$ for any $\mu \in(0, a)$ and

(ii) there is a constant $C_{1}$ such that $\frac{1}{\left|\xi^{\prime}(\mu)\right|} \leq C_{1}$ for $0<\mu<a$.

Then there exists a constant $C$ such that for all $f \in L^{2}(0, a)$,

$$
\|G f\|_{L^{2}\left(R^{+}\right)} \leq C\|f\|_{L^{2}(0, a)} .
$$

Proof. Let $\omega=\xi(\mu)$. Then $\mu=\xi^{-1}(\omega)$ and $d \omega=\xi^{\prime}(\mu) d \mu$ since $\xi(\mu)$ is invertible. In terms of the variable $\omega$,

$$
G f(x)=\int_{\xi(0)}^{\xi(a)} e^{i \omega x} f\left(\xi^{-1}(\omega)\right) \frac{1}{\xi^{\prime}\left(\xi^{-1}(\omega)\right)} d \omega .
$$

It follows from Plancherel's Theorem that

$$
\begin{aligned}
\|G f\|_{L^{2}\left(R^{+}\right)}^{2} & =\frac{1}{2 \pi} \int_{\xi(0)}^{\xi(a)} f\left(\xi^{-1}(\omega)\right)^{2}\left(\frac{1}{\xi^{\prime}\left(\xi^{-1}(\omega)\right)}\right)^{2} d \omega \\
& =\frac{1}{2 \pi} \int_{0}^{a}|f(\mu)|^{2} \frac{1}{\left|\xi^{\prime}(\mu)\right|} d \mu \\
& \leq \frac{C_{1}}{2 \pi} \int_{0}^{a}|f(\mu)|^{2} d \mu .
\end{aligned}
$$

The proof is complete.

The next step is to obtain estimates for the operators $W_{b}(t)$ and $U_{b}^{ \pm}(t)$ defined in Proposition 2.2. The first one is a standard energy inequality.

Lemma 3.3. Given $s \geq 0$, there exists a constant $C=C_{s}$ such that

$$
\sup _{t \geq 0}\left\|\left[W_{b}(t) h\right](\cdot)\right\|_{H_{x}^{s}\left(R^{+}\right)} \leq C\|h\|_{H^{\frac{s+1}{3}}\left(R^{+}\right)}
$$

for all $h \in H_{0}^{\frac{s+1}{3}}\left(R^{+}\right)$.

Proof. It suffices to establish the estimate for $U_{b}(t)$ and to consider only the cases where $s=n$ is an integer. The analogous result for non-integer values of $s$ may be obtained by standard interpolation theory. First, notice that

$$
\begin{aligned}
D_{x}^{n}\left[U_{b}(t) h\right](x)= & \frac{1}{2 \pi} \int_{1}^{\infty} e^{i\left(\mu^{3}-\mu\right) t} e^{\omega(\mu) x} \omega^{n}(\mu)\left(3 \mu^{2}-1\right) \\
& \times \int_{0}^{\infty} e^{-\left(\mu^{3}-\mu\right) \zeta i} h(\zeta) d \zeta d \mu \\
= & I I_{1}(x, t)+I I_{2}(x, t)
\end{aligned}
$$

with

$$
I I_{1}(x, t)=\frac{1}{2 \pi} \int_{2 / \sqrt{3}}^{+\infty} e^{i\left(\mu^{3}-\mu\right) t} e^{\omega(\mu) x} \omega^{n}(\mu)\left(3 \mu^{2}-1\right) \int_{0}^{\infty} e^{-\left(\mu^{3}-\mu\right) \zeta i} h(\zeta) d \zeta d \mu
$$

and

$$
I I_{2}(x, t)=\frac{1}{2 \pi} \int_{1}^{2 / \sqrt{3}} e^{i\left(\mu^{3}-\mu\right) t} e^{\omega(\mu) x} \omega^{n}(\mu)\left(3 \mu^{2}-1\right) \int_{0}^{\infty} e^{-\left(\mu^{3}-\mu\right) \zeta i} h(\zeta) d \zeta d \mu
$$


where $\omega(\mu)=-\frac{i \mu+\sqrt{3 \mu^{2}-4}}{2}$. Because of the obvious inequality

$$
\left|I I_{1}(x, t)\right| \leq C \int_{2 / \sqrt{3}}^{+\infty} e^{-\frac{\sqrt{3 \mu^{2}-4}}{2} x}|\omega(\mu)|^{n}\left(3 \mu^{2}-1\right)\left|\int_{0}^{\infty} e^{-\left(\mu^{3}-\mu\right) \zeta i} h(\zeta) d \zeta\right| d \mu
$$

it follows by Lemma 3.1 that

$$
\begin{aligned}
& \sup _{t \geq 0}\left\|I I_{1}(\cdot, t)\right\|_{L_{x}^{2}\left(R^{+}\right) \leq C \|} \int_{2 / \sqrt{3}}^{+\infty} e^{-\frac{\sqrt{3 \mu^{2}-4}}{2} x}(1+\mu)^{2+n} \\
& \quad \times\left|\int_{0}^{\infty} e^{-\left(\mu^{3}-\mu\right) \zeta i} h(\zeta) d \zeta\right| d \mu \|_{L_{x}^{2}\left(R^{+}\right)} \\
& \leq C\left(\int_{1}^{\infty}(1+\mu)^{4+2 n}\left|\int_{0}^{\infty} e^{-\left(\mu^{3}-\mu\right) \zeta i} h(\zeta) d \zeta\right|^{2} d \mu\right)^{1 / 2} \\
& \leq C\left(\int_{0}^{\infty}(1+\eta)^{2(n+1) / 3}\left|\int_{0}^{\infty} e^{-\eta \zeta i} h(\zeta) d \zeta\right|^{2} d \eta\right)^{1 / 2} \\
& \leq C\|h\|_{H^{(n+1) / 3}\left(R^{+}\right)} .
\end{aligned}
$$

As for $I I_{2}(x, t)$, note that $\omega(\mu) / i$ is real when $1 \leq \mu \leq 2 / \sqrt{3}$. Applying Lemma 3.2 directly to $I_{2}(x, t)$ yields that

$$
\begin{aligned}
& \sup _{t \geq 0}\left\|I I_{2}(\cdot, t)\right\|_{L_{x}^{2}\left(R^{+}\right)} \leq C\left(\int_{1}^{2 / \sqrt{3}}(1+\mu)^{4+2 n}\left|\int_{0}^{\infty} e^{-\left(\mu^{3}-\mu\right) \zeta i} h(\zeta) d \zeta\right|^{2} d \mu\right)^{1 / 2} \\
& \quad \leq C_{n}\left(\int_{0}^{\infty}\left|\int_{0}^{\infty} e^{-\eta \zeta i} h(\zeta) d \zeta\right|^{2} d \eta\right)^{1 / 2} \\
& \leq C_{n}\|h\|_{L^{2}\left(R^{+}\right)}
\end{aligned}
$$

Consequently

$$
\sup _{t \geq 0}\left\|\left[U_{b}(t) h\right](\cdot)\right\|_{H_{x}^{n}\left(R^{+}\right)} \leq C_{n}\|h\|_{H^{\frac{n+1}{3}}\left(R^{+}\right)} .
$$

The proof is complete.

The following inequality comprises a sharp version of the Kato smoothing property (see Kato [37] 38], Kruzhkov and Faminskii [45, 46]) for the semigroup $W_{b}(t)$.

Lemma 3.4. For any $s \geq 0$, there exists a constant $C=C_{s}$ such that

$$
\sup _{x \in R^{+}}\left(\int_{0}^{\infty}\left(J_{x}^{s+1}\left[W_{b}(t) h\right](x)\right)^{2} d t\right)^{1 / 2} \leq C\|h\|_{H^{\frac{s+1}{3}}\left(R^{+}\right)}
$$

for all $h \in H_{0}^{\frac{s+1}{3}}\left(R^{+}\right)$.

Proof. We prove the estimate for $0 \leq s<1$. The proof for other values of $s$ is similar. Consider first $U_{b}(t) h$. Let $\eta=\mu^{3}-\mu$ for $\mu \geq 1$ and, for $\eta \geq 0$, let $\mu=\delta(\eta)$ be the unique real solution of

$$
\eta=\mu^{3}-\mu
$$


Note that

$$
\begin{aligned}
D_{x}\left[U_{b}(t) h\right](x) & =\frac{1}{2 \pi} \int_{1}^{\infty} \omega(\mu) e^{i \mu^{3} t-i \mu t} e^{\omega(\mu) x}\left(3 \mu^{2}-1\right) \int_{0}^{\infty} e^{-\left(\mu^{3}-\mu\right) \xi i} h(\xi) d \xi d \mu \\
& =\frac{1}{2 \pi} \int_{0}^{\infty} \omega(\delta(\eta)) e^{i \eta t} e^{\omega(\delta(\eta)) x} \int_{0}^{\infty} e^{-\eta \xi i} h(\xi) d \xi d \eta
\end{aligned}
$$

Using the Plancherel Theorem with respect to $t$ in the above representation of $D_{x}\left(U_{b}(t) h\right)$, one sees that

$$
\int_{0}^{\infty}\left|D_{x}\left[U_{b}(t) h\right](x)\right|^{2} d t \leq C \int_{0}^{\infty}(1+\eta)^{2 / 3}\left|\int_{0}^{\infty} e^{-\eta \xi i} h(\xi) d \xi\right|^{2} d \eta
$$

for any $x \in R^{+}$. Thus the lemma holds for $s=0$. For $0<s<1$, since

$$
\begin{gathered}
\left|J_{x}^{1+s}\left[U_{b}(t) h\right](x)\right|^{2}=\frac{1}{2 \pi} \mid J_{x}^{s} \int_{1}^{\infty} \omega(\mu) e^{i \mu^{3} t-i \mu t} e^{\omega(\mu) x}\left(3 \mu^{2}-1\right) \\
\times\left.\int_{0}^{\infty} e^{-i\left(\mu^{3}-\mu\right) \xi} h(\xi) d \xi d \mu\right|^{2} \\
=\frac{1}{2 \pi} \int_{0}^{\infty} \tau^{-(2 s+1)} \mid \int_{1}^{\infty} \omega(\mu) e^{i \mu^{3} t-i \mu t} e^{\omega(\mu) x}\left(e^{\omega(\mu) \tau}-1\right) \\
\times\left.\left(3 \mu^{2}-1\right) \int_{0}^{\infty} e^{-i\left(\mu^{3}-\mu\right) \xi} h(\xi) d \xi d \mu\right|^{2} d \tau \\
=\left.\frac{1}{2 \pi} \int_{0}^{\infty} \tau^{-(2 s+1)}\right|_{1} ^{\infty} \omega(\delta(\eta)) e^{i \eta t} e^{\omega(\delta(\eta)) x}\left(e^{\omega(\delta(\eta)) \tau}-1\right) \\
\times\left.\int_{0}^{\infty} e^{-i \eta \xi} h(\xi) d \xi d \mu\right|^{2} d \tau,
\end{gathered}
$$

the Plancherel Theorem may be used as before to adduce

$$
\begin{aligned}
& \int_{0}^{\infty}\left|J_{x}^{1+s}\left[U_{b}(t) h\right](x)\right|^{2} d t \\
\leq & C \int_{0}^{\infty} \tau^{-(2 s+1)} \int_{0}^{\infty}\left|e^{\omega(\delta(\eta)) \tau}-1\right|^{2}\left|\omega(\delta(\eta)) \int_{0}^{\infty} e^{-i \eta \xi} h(\xi) d \xi\right|^{2} d \eta d \tau \\
\leq & C \int_{0}^{\infty} \int_{0}^{\infty} \tau^{-(2 s+1)}\left|e^{\omega(\delta(\eta)) \tau}-1\right|^{2} d \tau\left|\omega(\delta(\eta)) \int_{0}^{\infty} e^{-i \eta \xi} h(\xi) d \xi\right|^{2} d \eta \\
\leq & C \int_{0}^{\infty} y^{-(2 s+1)}\left|e^{-y}-1\right|^{2} d y \int_{0}^{\infty}|\omega(\delta(\eta))|^{2(s+1)}\left|\int_{0}^{\infty} e^{-i \eta \xi} h(\xi) d \xi\right|^{2} d \eta \\
\leq & C \int_{0}^{\infty}(1+\eta)^{2(s+1) / 3}\left|\int_{0}^{\infty} e^{-i \eta \xi} h(\xi) d \xi\right|^{2} d \eta \leq C|| h \|_{H^{(1+s) / 3}\left(R^{+}\right)} .
\end{aligned}
$$

It is thus proved that the lemma is true for $0 \leq s<1$.

Solutions of the linear $\mathrm{KdV}$ equation

$$
u_{t}+u_{x}+u_{x x x}=0,
$$


have the formal property that temporal derivatives are balanced by three times as many spatial derivatives, viz.,

$$
D_{t}^{s} \sim D_{x}^{3 s}
$$

for $s>0$. The following lemma gives precision to this observation.

Lemma 3.5. For any $s \geq 0$, there exists a constant $C=C_{s}$ such that

$$
\sup _{x \in R^{+}}\left\|D_{x}^{k}\left[W_{b}(\cdot) h\right](x)\right\|_{H_{t}^{\frac{s-k+1}{3}}\left(R^{+}\right)} \leq C\|h\|_{H^{\frac{s+1}{3}}\left(R^{+}\right)}
$$

for $k=0,1, \cdots,[s]$ and all $h \in H_{0}^{\frac{s+1}{3}}\left(R^{+}\right)$.

Proof. It suffices to show that (3.6) holds for $\left[U_{b}(t) h\right](x)$. A change of variables as in Lemma 3.4 gives

$$
\begin{gathered}
D_{x}^{k}\left[U_{b}(t) h\right](x)=\frac{1}{2 \pi} \int_{1}^{\infty} e^{i\left(\mu^{3}-\mu\right) t} \omega^{k}(\mu) e^{\omega(\mu) x}\left(3 \mu^{2}-1\right) \\
\times \int_{0}^{\infty} e^{-i\left(\mu^{3}-\mu\right) \xi} h(\xi) d \xi d \mu \\
=\frac{1}{2 \pi} \int_{0}^{\infty} e^{i \eta t} \omega^{k}(\delta(\eta)) e^{\omega(\delta(\eta)) x} \int_{0}^{\infty} e^{-i \eta \xi} h(\xi) d \xi d \eta,
\end{gathered}
$$

where $\delta(\eta)$ is specified in the last proof. It follows from arguments, by now familiar, that for any $x \geq 0$,

$$
\begin{aligned}
\left\|D_{x}^{k}\left[U_{b}(\cdot) h\right](x)\right\|_{H_{t}^{\frac{s-k+1}{3}}}^{2}\left(R^{+}\right) \\
\quad \leq C \int_{0}^{\infty}(1+\eta)^{\frac{2(s-k+1)}{3}}|\omega(\delta(\eta))|^{2 k}\left|\int_{0}^{\infty} e^{-i \eta \xi} h(\xi) d \xi\right|^{2} d \eta \\
\quad \leq C \int_{0}^{\infty}(1+\eta)^{\frac{2(s+1)}{3}}\left|\int_{0}^{\infty} e^{-i \eta \xi} h(\xi) d \xi\right|^{2} d \eta=C\|h\|_{H^{\frac{s+1}{3}}\left(R^{+}\right)} .
\end{aligned}
$$

Here we note that we can take $t$-derivatives of $U_{b}(t) h$ of any fractional order directly using Fourier transforms and cutoff functions. The proof is completed.

Lemma 3.6. For any non-negative integer $n$, there exists a constant $C_{n}$ such that

$$
\left(\int_{0}^{\infty} \sup _{t \geq 0}\left|D_{x}^{n}\left[W_{b}(t) h\right](x)\right|^{2} d x\right)^{1 / 2} \leq C_{n}\|h\|_{H^{(n+1) / 3}\left(R^{+}\right)}
$$

for all $h \in H_{0}^{(n+1) / 3}\left(R^{+}\right)$.

Proof. The proof is given in detail for $U_{b}(t) h$. As in the proof of Lemma 3.3,

$$
D_{x}^{n}\left[U_{b}(t) h\right](x)=I I_{1}(x, t)+I I_{2}(x, t)
$$


where $I I_{1}(x, t)$ and $I I_{2}(x, t)$ are defined in (3.3) and (3.4), respectively. By the inequality (3.5), it follows from Lemma 3.1 that

$$
\begin{aligned}
& \left(\int_{0}^{\infty} \sup _{t \geq 0}\left|I I_{1}(x, t)\right|^{2} d x\right)^{1 / 2} \\
& \quad \leq C\left(\int_{0}^{\infty}|\omega(\mu)|^{2 n}\left(3 \mu^{2}-1\right)^{2}\left|\int_{0}^{\infty} e^{-\left(\mu^{3}-\mu\right) \zeta i} h(\zeta) d \zeta\right|^{2} d \mu\right)^{1 / 2} \\
& \quad \leq C\left(\int_{0}^{\infty}(1+\eta)^{2(n+1) / 3}\left|\int_{0}^{\infty} e^{-\eta \zeta i} h(\zeta) d \zeta\right|^{2} d \eta\right)^{1 / 2} \\
& \quad=C\|h\|_{H^{(n+1) / 3}\left(R^{+}\right)} .
\end{aligned}
$$

As for $I I_{2}(x, t)$, recall $\omega(\mu)$ is purely imaginary for $1 \leq \mu \leq 2 / \sqrt{3}$. If we make the change of variables from $\mu$ to $y$, where

$$
y=\omega(\mu) / i=-\frac{\mu+\sqrt{4-3 \mu^{2}}}{2},
$$

in the representation of $I I_{2}(x, t)$, there appears the formula

$$
\begin{aligned}
I I_{2}(x, t) & =\frac{1}{2 \pi} \int_{1}^{2 / \sqrt{3}} e^{i \mu^{3} t-i \mu t} e^{\omega(\mu) x}\left(3 \mu^{2}-1\right) \int_{0}^{\infty} e^{-i\left(\mu^{3}-\mu\right) \xi} h(\xi) d \xi d \mu \\
& =\frac{1}{2 \pi} \int_{-1}^{-1 / \sqrt{3}} e^{i y^{3} t-i y t} e^{i y x}\left(3 y^{2}-1\right) \int_{0}^{\infty} e^{-i \eta(y) \xi} h(\xi) d \xi d y \\
& =\frac{1}{2 \pi} \int_{-\infty}^{+\infty} e^{i y^{3} t-i y t} e^{i y x} \hat{g}(y) d y
\end{aligned}
$$

where $\mu=\eta(y)$ is the unique real solution of the equation (3.7) in the range $[1,2 / \sqrt{3}], g$ is the inverse Fourier transform of the function

$$
q(y)=\chi(y) \frac{3 y^{2}-1}{3 \eta^{2}(y)-1} \int_{0}^{\infty} e^{-i \eta(y) \xi} \psi(\xi) d \xi,
$$

and $\chi$ is the characteristic function of the interval $(-1,-1 / \sqrt{3})$. Applying Corollary 2.9 of [41] yields that, for a given $s>3 / 4$, there exists a constant $C=C_{s}$ such that

$$
\left(\int_{0}^{\infty} \sup _{t \geq 0}\left|I I_{2}(x, t)\right|^{2} d x\right)^{1 / 2} \leq C\|g\|_{H^{s}(R)}
$$

Since

$$
\begin{aligned}
\|g\|_{H^{s}(R)}^{2} & =\int_{-\infty}^{+\infty}\left(1+y^{2}\right)^{s}\left|\chi(y) i^{n} y^{n}\left(3 y^{2}-1\right) \int_{0}^{\infty} e^{\left(y^{3}-y\right) \xi i} h(\xi) d \xi\right|^{2} d y \\
& =\int_{-1}^{-\frac{1}{\sqrt{3}}}\left(1+y^{2}\right)^{s} y^{2 n}\left(3 y^{2}-1\right)^{2}\left|\int_{0}^{\infty} e^{\left(y^{3}-y\right) \xi i} h(\xi) d \xi\right|^{2} d y \\
& \leq C_{n}\|h\|_{L^{2}\left(R^{+}\right)},
\end{aligned}
$$


one obtains

$$
\left(\int_{0}^{\infty} \sup _{t \geq 0}\left|I I_{2}(x, t)\right|^{2} d x\right)^{1 / 2} \leq C_{n}\|h\|_{L^{2}\left(R^{+}\right)} .
$$

The estimate for $U_{b}(t) h$ is thus established. The proof is complete.

Next is presented a half-line version of Theorem 2.4 in 41 which reveals a global smoothing effect of Strichartz type for the semigroup $W_{b}(t)$. We first consider the operator $\mathcal{U}(t)$ defined by

$$
\mathcal{U}(t) \psi(x)=\frac{1}{2 \pi} \int_{1}^{+\infty} e^{i \mu^{3} t-i \mu t} e^{\omega(\mu) x} \int_{-\infty}^{\infty} e^{-i \mu \xi} \psi(\xi) d \xi d \mu
$$

for any $\psi \in L^{2}(R)$.

Lemma 3.7. For any $(\theta, \beta) \in[0,1] \times[0,1 / 2]$ and any $T>0$, there exists a constant $C_{T}$ such that

$$
\left(\int_{0}^{T}\left\|\mathcal{D}^{\theta \beta / 2} \mathcal{U}(t) \psi\right\|_{L^{p}\left(R^{+}\right)}^{q} d t\right)^{1 / q} \leq C_{T}\|\psi\|_{L^{2}(R)}
$$

for any $\psi \in L^{2}(R)$ where

$$
(q, p)=\left(\frac{6}{\theta(\beta+1)}, \frac{2}{1-\theta}\right) .
$$

Here, by definition, for $r \geq 0$,

$$
\mathcal{D}^{r} \mathcal{U}(t) \psi(x)=\frac{1}{2 \pi} \int_{1}^{\infty} \mu^{r} e^{i \mu^{3} t-i \mu t} e^{\omega(\mu) x} \int_{-\infty}^{\infty} e^{-i \mu \xi} \psi(\xi) d \xi d \mu .
$$

Proof. Rewrite $\mathcal{U}(t) \psi$ as $\mathcal{U}^{+}(t) \psi+\mathcal{U}^{-}(t) \psi$ with

$$
\mathcal{U}^{+}(t) \psi(x)=\frac{1}{2 \pi} \int_{1}^{+\infty} e^{i \mu^{3} t-i \mu t} e^{\omega(\mu) x} \int_{0}^{\infty} e^{-i \mu \xi} \psi(\xi) d \xi d \mu
$$

and

$$
\mathcal{U}^{-}(t) \psi(x)=\frac{1}{2 \pi} \int_{1}^{+\infty} e^{i \mu^{3} t-i \mu t} e^{\omega(\mu) x} \int_{-\infty}^{0} e^{-i \mu \xi} \psi(\xi) d \xi d \mu .
$$

The estimate (3.8) for $\mathcal{U}^{+}(t) \psi$ is established in detail. The proof for $\mathcal{U}^{-}(t) \psi$ is similar. Write $\mathcal{U}^{+}(t) \psi(x)$ as

$$
\mathcal{U}^{+}(t) \psi(x)=\mathcal{U}_{1}(t) \psi(x)+\mathcal{U}_{2}(t) \psi(x)+\mathcal{U}_{3}(t) \psi(x)
$$

with

$$
\begin{aligned}
& \mathcal{U}_{1}(t) \psi(x)=\frac{1}{2 \pi} \int_{1}^{2 / \sqrt{3}} e^{i \mu^{3} t-i \mu t} e^{\omega(\mu) x} \int_{0}^{\infty} e^{-i \mu \xi} \psi(\xi) d \xi d \mu \\
& \mathcal{U}_{2}(t) \psi(x)=\frac{1}{2 \pi} \int_{2 / \sqrt{3}}^{2} e^{i \mu^{3} t-i \mu t} e^{\omega(\mu) x} \int_{0}^{\infty} e^{-i \mu \xi} \psi(\xi) d \xi d \mu
\end{aligned}
$$

and

$$
\mathcal{U}_{3}(t) \psi(x)=\frac{1}{2 \pi} \int_{2}^{+\infty} e^{i \mu^{3} t-i \mu t} e^{\omega(\mu) x} \int_{0}^{\infty} e^{-i \mu \xi} \psi(\xi) d \xi d \mu .
$$


To prove estimate (3.8) it suffices to show that

$$
\left(\int_{0}^{T}\left\|\mathcal{D}^{\theta \beta / 2} \mathcal{U}_{j}(t) \psi\right\|_{L^{p}\left(R^{+}\right)}^{q} d t\right)^{1 / q} \leq C_{T}\|\psi\|_{L^{2}\left(R^{+}\right)}
$$

for $j=1,2,3$.

For $\mathcal{U}_{1}(t) \psi$, the argument appearing in the proof of Lemma 3.6 shows this quantity can be written in the form

$$
\begin{aligned}
\mathcal{U}_{1}(t) \psi(x) & =\frac{1}{2 \pi} \int_{1}^{2 / \sqrt{3}} e^{i \mu^{3} t-i \mu t} e^{\omega(\mu) x} \int_{0}^{\infty} e^{-i \mu \xi} \psi(\xi) d \xi d \mu \\
& =\frac{1}{2 \pi} \int_{-1}^{-1 / \sqrt{3}} e^{i y^{3} t-i y t} e^{i y x} \frac{3 y^{2}-1}{3 \eta^{2}(y)-1} \int_{0}^{\infty} e^{-i \eta(y) \xi} \psi(\xi) d \xi d y \\
& =\frac{1}{2 \pi} \int_{-\infty}^{+\infty} e^{i y^{3} t-i y t} e^{i y x} \hat{g}(y) d y
\end{aligned}
$$

where $g$ is the inverse Fourier transform of the function

$$
q(y)=\chi(y) \frac{3 y^{2}-1}{3 \eta^{2}(y)-1} \int_{0}^{\infty} e^{-i \eta(y) \xi} \psi(\xi) d \xi
$$

and $\chi$ is the characteristic function of the interval $(-1,-1 / \sqrt{3})$. Applying Theorem 2.4 of 41 yields

$$
\left(\int_{0}^{\infty}\left\|\mathcal{D}^{\frac{\theta \beta}{2}} \mathcal{U}_{1}(t) \psi\right\|_{L_{x}^{p}\left(R^{+}\right)}^{q} d t\right)^{1 / q} \leq C\|g\|_{L^{2}(R)} \leq C\|\psi\|_{L^{2}\left(R^{+}\right)}
$$

For $\mathcal{U}_{2}(t) \psi$, we have

$$
\begin{aligned}
& \left\|\mathcal{D}^{\frac{\theta \beta}{2}} \mathcal{U}_{2}(t) \psi\right\|_{L_{x}^{2}\left(R^{+}\right)} \leq C \int_{\frac{2}{\sqrt{3}}}^{2} \frac{1}{\left(\mu-\frac{2}{\sqrt{3}}\right)^{\frac{1}{2 p}}}\left|\int_{0}^{\infty} e^{-i \mu \xi} \psi(\xi) d \xi\right| d \mu \\
& \quad \leq C\left(\int_{\frac{2}{\sqrt{3}}}^{2} \frac{1}{\left(\mu-\frac{2}{\sqrt{3}}\right)^{\frac{1}{p}}} d \mu\right)^{1 / 2}\left(\int_{\frac{2}{\sqrt{3}}}^{2}\left|\int_{0}^{\infty} e^{-i \mu \xi} \psi(\xi) d \xi\right|^{2} d \mu\right)^{1 / 2} \\
& \leq C\|\phi\|_{L^{2}\left(R^{+}\right)}
\end{aligned}
$$

for any $t \geq 0$. Thus we arrive at

$$
\left(\int_{0}^{T}\left\|\mathcal{D}^{\frac{\theta \beta}{2}} \mathcal{U}_{2}(t) \psi\right\|_{L_{x}^{p}\left(R^{+}\right)}^{q} d t\right)^{1 / q} \leq C T\|\psi\|_{L^{2}\left(R^{+}\right)} .
$$

On the other hand, the inequality in (3.9) with $j=3$ is equivalent by duality to the inequality

$$
\left\|\int_{0}^{\infty} \mathcal{D}^{\theta \beta / 2} \mathcal{U}_{3}(t) f(\cdot, t) d t\right\|_{L^{2}\left(R^{+}\right)} \leq C\left(\int_{0}^{\infty}\|f(\cdot, t)\|_{L^{p^{\prime}}}^{q^{\prime}} d t\right)^{\frac{1}{q^{\prime}}}
$$

where

$$
\frac{1}{p}+\frac{1}{p^{\prime}}=\frac{1}{q}+\frac{1}{q^{\prime}}=1
$$


Observe that

$$
\begin{aligned}
& \left\|\int_{0}^{\infty} \mathcal{D}^{\theta \beta / 2} \mathcal{U}_{3}(t) f(\cdot, t) d t\right\|_{L^{2}\left(R^{+}\right)}^{2} \\
& =\int_{0}^{\infty} \int_{0}^{\infty} f\left(\zeta_{1}, t\right) \int_{0}^{\infty} \int_{0}^{\infty} \overline{f\left(\zeta_{2}, \tau\right)} H\left(\zeta_{1}, \zeta_{2}, t, \tau\right) d \zeta_{2} d \tau d \zeta_{1} d t
\end{aligned}
$$

with

$$
\begin{gathered}
H\left(\zeta_{1}, \zeta_{2}, t, \tau\right) \\
=\int_{2}^{+\infty} \int_{2}^{+\infty} e^{i \mu_{1}^{3} t-i \mu_{1} t-i \mu_{2}^{3} \tau+i \mu_{2} \tau} e^{-\mu_{1} \zeta_{1} i+\mu_{2} \zeta_{2} i}\left(\mu_{1} \mu_{2}\right)^{\theta \beta / 2} \\
\times \int_{0}^{\infty} e^{\left(\omega\left(\mu_{1}\right)+\overline{\omega\left(\mu_{2}\right)}\right) x} d x d \mu_{1} d \mu_{2} \\
=\int_{2}^{+\infty} \int_{2}^{+\infty} e^{i \mu_{1}^{3} t-i \mu_{1} t-i \mu_{2}^{3} \tau i+i \mu_{2} \tau} e^{-\mu_{1} \zeta_{1} i+\mu_{2} \zeta_{2} i} \frac{\left(\mu_{1} \mu_{2}\right)^{\theta \beta / 2}}{-\omega\left(\mu_{1}\right)-\overline{\omega\left(\mu_{2}\right)}} d \mu_{1} d \mu_{2}
\end{gathered}
$$

and, as in (3.5),

$$
\omega(\mu)=-\frac{i \mu+\sqrt{3 \mu^{2}-4}}{2} .
$$

Again appealing to duality, it suffices to show that there is a constant $C$ such that

$$
\left\|\int_{0}^{\infty}\right\| \int_{0}^{\infty} \overline{f\left(\zeta_{2}, \tau\right)} H\left(\zeta_{1}, \zeta_{2}, t, \tau\right) d \zeta_{2}\left\|_{L_{\zeta_{1}}^{p}}^{q} d \tau\right\|_{L_{t}^{q}} \leq C\left(\int_{0}^{\infty}\|f(\cdot, t)\|_{L^{p^{\prime}}}^{q^{\prime}} d t\right)^{\frac{1}{q^{\prime}}}
$$

To this end, first change variables to derive the inequality

$$
\begin{aligned}
& \left|\int_{0}^{\infty} \overline{f\left(\zeta_{2}, \tau\right)} H\left(\zeta_{1}, \zeta_{2}, t, \tau\right) d \zeta_{2}\right| \\
= & \mid \int_{0}^{\infty} \overline{f\left(\zeta_{2}, \tau\right)} \\
& \times\left(\int_{2}^{+\infty} d \mu_{1} \int_{2 / \mu_{1}}^{\infty} \frac{\mu_{1}^{\theta \beta} y^{\frac{\theta \beta}{2}} e^{i \mu_{1}^{3}\left(t-y^{3} \tau\right)-i \mu_{1}(t-y \tau)-i \mu_{1}\left(\zeta_{1}-\zeta_{2} y\right)}}{\mu_{1}^{-1} \omega\left(\mu_{1}\right)+\mu_{1}^{-1} \overline{\omega\left(\mu_{1} y\right)}} d y\right) d \zeta_{2} \mid \\
\leq & C \int_{0}^{\infty} \frac{y^{\frac{\theta \beta}{2}}}{1+y} \int_{0}^{\infty} d \mu_{1} \mu_{1}^{\theta \beta} e^{i \mu_{1}^{3}\left(t-y^{3} \tau\right)-i \mu_{1}(t-y \tau)-i \mu_{1}\left(\zeta_{1}-\zeta_{2} y\right)} \overline{f\left(\zeta_{2}, \tau\right)} d \zeta_{2} \mid d y .
\end{aligned}
$$


It follows from this that

$$
\begin{aligned}
& I I I_{1}(t, \tau) \equiv\left\|\int_{0}^{\infty} \overline{f\left(\zeta_{2}, \tau\right)} H\left(\zeta_{1}, \zeta_{2}, t, \tau\right) d \zeta_{2}\right\|_{L_{\zeta_{1}}^{p}} \\
\leq \quad & \| \int_{0}^{\infty} \frac{y^{\frac{\theta \beta}{2}}}{1+y} \\
& \times\left|\int_{2 / y}^{\infty} d \mu_{1} \int_{0}^{\infty} \mu_{1}^{\theta \beta} e^{i \mu_{1}^{3}\left(t-y^{3} \tau\right)-i \mu_{1}(t-y \tau)-i \mu_{1}\left(\zeta_{1}-\zeta_{2} y\right)} \overline{f\left(\zeta_{2}, \tau\right)} d \zeta_{2}\right| d y \|_{L_{\zeta_{1}}^{p}} \\
\leq \quad & \int_{0}^{\infty} \frac{y^{\frac{\theta \beta}{2}}}{1+y} \\
\quad & \left\|\int_{2 / y}^{\infty} \int_{0}^{\infty} \mu_{1}^{\theta \beta} e^{i \mu_{1}^{3}\left(t-y^{3} \tau\right)-i \mu_{1}(t-y \tau)-i \mu_{1}\left(\zeta_{1}-\zeta_{2} y\right)} \overline{f\left(\zeta_{2}, \tau\right)} d \zeta_{2} d \mu_{1}\right\|_{L_{\zeta_{1}}^{p}} d y .
\end{aligned}
$$

If the inequality

$$
\begin{gathered}
\left\|\int_{2 / y}^{\infty} \int_{0}^{\infty} \overline{f(z, \tau)} \mu_{1}^{\theta \beta} e^{i \mu_{1}^{3}\left(t-y^{3} \tau\right)-i \mu_{1}(t-y \tau)-i \mu_{1}\left(\zeta_{1}-z\right)} d \mu_{1} d z\right\|_{L_{\zeta_{1}}^{p}} \\
\quad \leq C\left|t-y^{3} \tau\right|^{-\frac{\theta(\beta+1)}{3}}\|f(\cdot, \tau)\|_{L^{p^{\prime}}}
\end{gathered}
$$

can be proved, then the preceding inequality gives

$$
I I I_{1}(t, \tau) \leq C \int_{0}^{\infty} \frac{y^{\frac{\theta \beta}{2}-\frac{1}{p}}}{1+y}\left|t-y^{3} \tau\right|^{-\frac{\theta(\beta+1)}{3}} d y\|f(\cdot, \tau)\|_{L^{p^{\prime}}}
$$

from which it follows that

$$
\begin{aligned}
\| \int_{0}^{\infty} & \operatorname{III}_{1}(\cdot, \tau) d \tau \|_{L_{t}^{q}} \\
\leq & \int_{0}^{\infty} \frac{y^{\frac{\theta \beta}{2}-\frac{1}{p}}}{1+y}\left\|\int_{0}^{\infty}\left|t-y^{3} \tau\right|^{-\frac{\theta(\beta+1)}{3}}\right\| f(\cdot, \tau)\left\|_{L^{p^{\prime}}} d \tau\right\|_{L_{t}^{q}} d y \\
= & C \int_{0}^{\infty} \frac{y^{\frac{\theta \beta}{2}-\frac{1}{p}}}{1+y} y^{\frac{3}{q}-\theta(\beta+1)} \\
& \times\left(\int_{0}^{\infty}\left(\int_{0}^{\infty}|\xi-\tau|^{-\frac{\theta(\beta+1)}{3}}\|f(\cdot, \tau)\|_{L^{p^{\prime}}} d \tau\right)^{q} d \xi\right)^{1 / q} d y
\end{aligned}
$$


provided $q$ is finite. This inequality may be extended using a classical integral inequality (cf. Hardy, Littlewood and Polya [31], Th. 382, p. 288), viz.

$$
\begin{aligned}
\left\|\int_{0}^{+\infty} I I I_{1}(\cdot, \tau) d \tau\right\|_{L_{t}^{q}} & \leq C \int_{0}^{\infty} \frac{y^{\frac{\theta \beta}{2}-\frac{1}{p}+\frac{3}{q}-\theta(\beta+1)}}{1+y} d y\left(\int_{0}^{\infty}\|f(\cdot, t)\|_{L^{p^{\prime}}}^{q^{\prime}} d t\right)^{\frac{1}{q^{\prime}}} \\
& =C \int_{0}^{\infty} \frac{1}{y^{1 / 2}(1+y)} d y\left(\int_{0}^{\infty}\|f(\cdot, t)\|_{L^{p^{\prime}}}^{q^{\prime}} d t\right)^{\frac{1}{q^{\prime}}} \\
& \leq C\left(\int_{0}^{\infty}\|f(\cdot, t)\|_{L^{p^{\prime}}}^{q^{\prime}} d t\right)^{\frac{1}{q^{\prime}}} .
\end{aligned}
$$

If $q=\infty, \theta=0$, so $p=2$ and the result follows by a separate, but easier argument.

Thus it remains to establish the estimate (3.10) to complete the proof. To accomplish this it suffices to verify the following claim which yields estimate (3.10) by simply taking

$$
\alpha=\beta, \quad T=t-y \tau, \quad t=t-y^{3} \tau,
$$

and $\phi=\overline{f(z, \tau)}$ in the claim.

Claim. For given $y>0, T \in R$ and $(\theta, \alpha) \in[0,1] \times[0,1 / 2]$, define

$$
S_{\theta \alpha}(x, t)=\int_{2 / y}^{\infty} \mu^{\alpha \theta} e^{\mu^{3} i t} e^{-i \mu T} e^{i \mu x} d \mu
$$

and, for $\phi \in H^{2 /(1+\theta)}$,

$$
L_{\theta \alpha}(t) \phi=S_{\theta \alpha}(\cdot, t) * \phi .
$$

It follows that for any $t>0$,

$$
\left\|L_{\theta \alpha}(t) \phi\right\|_{2 /(1-\theta)} \leq C t^{-\theta(\alpha+1) / 3}\|\phi\|_{2 /(1+\theta)}
$$

where $C$ is independent of $t, y$ and $T$.

To see if the claim is true, introduce the analytic family of operators

$$
\begin{aligned}
& L_{\alpha+i \beta}(t) \phi=S_{\alpha+i \beta}(\cdot, t) * \phi \\
& \quad=\int_{2 / y}^{\infty} \mu^{\alpha+i \beta} e^{\mu^{3} i t} e^{-i \mu T} \int_{0}^{\infty} e^{-i \mu(x-\zeta)} \phi(\zeta) d \zeta d \mu
\end{aligned}
$$

for $(\alpha, \beta) \in[0,1 / 2] \times R$. First, it is straightforward to determine that

$$
\left\|L_{i \beta}(t) \phi\right\|_{L^{2}\left(R^{+}\right)} \leq C\|\phi\|_{L^{2}\left(R^{+}\right)}
$$

for a constant $C$ which is independent of $t, T, y$ and $\beta$. Using the argument appearing in the proof of Lemma 2.1 in [39] yields

$$
\left|S_{\alpha+i \beta}(x, t)\right| \leq C(1+|\beta|) t^{-(\alpha+1) / 3}
$$

for any $x \geq 0$ and $t>0$, where the constant $C$ is again independent of $t, y$ and $T$. As a result, for $\alpha \in[0,1 / 2]$, there obtains

$$
\left\|L_{\alpha+i \beta}(t) \phi\right\|_{L^{\infty}\left(R^{+}\right)} \leq C t^{-(\alpha+1) / 3}\|\phi\|_{L^{1}\left(R^{+}\right)} .
$$

Estimate (3.11) is obtained by a straightforward complex interpolation (see [55] Chapter V, Theorem 41). The proof is complete.

As a corollary to Lemma 3.7, there follows some related inequalities. 
Lemma 3.8. Given $s \geq 0$, there exists a constant $C=C_{s}$ such that

$$
\left(\int_{0}^{\infty} \sup _{x \in R^{+}}\left|\mathcal{D}^{s+1 / 4}\left[W_{b}(t) h\right](x)\right|^{4} d t\right)^{1 / 4} \leq C\|h\|_{H^{\frac{s}{3}}\left(R^{+}\right)}
$$

for all $h \in H_{0}^{\frac{s}{3}}\left(R^{+}\right)$. Here, by definition, for $r \geq 0$,

$$
\mathcal{D}^{r} W_{b}(t) h=\mathcal{D}^{r} U_{b}(t) h+\overline{\mathcal{D}^{r} U_{b}(t) h}
$$

with

$$
\left[\mathcal{D}^{r} U_{b}(t) h\right](x)=\frac{1}{2 \pi} \int_{1}^{\infty} e^{i\left(\mu^{3}-\mu\right) t} \mu^{r} e^{\omega(\mu) x}\left(3 \mu^{2}-1\right) \int_{0}^{\infty} e^{-i\left(\mu^{3}-\mu\right) \xi} h(\xi) d \xi d \mu .
$$

In particular, for any integer $n \geq 1$, there corresponds a constant $C=C_{n}$ such that

$$
\left(\int_{0}^{\infty} \sup _{x \in R^{+}}\left|D_{x}^{n}\left[W_{b}(t) h\right](x)\right|^{4} d t\right)^{1 / 4} \leq C\|h\|_{H^{\frac{4 n-1}{12}}\left(R^{+}\right)}
$$

for all $h \in H_{0}^{\frac{4 n-1}{12}}\left(R^{+}\right)$.

Proof. We write $\mathcal{D}^{s} U_{b}(t) h$ as

$$
\left[\mathcal{D}^{s} U_{b}(t) h\right](x)=\frac{1}{2 \pi} \int_{1}^{\infty} e^{i \mu^{3} t-i \mu t} e^{\omega(\mu) x} \hat{f}(\mu) d \mu=\mathcal{U}(t) f(x)
$$

where the Fourier transform of $f$ is

$$
\hat{f}(\mu)=|\mu|^{s}\left(3 \mu^{2}-1\right) \int_{0}^{\infty} e^{-\left(\mu^{3}-\mu\right) i \zeta} h(\zeta) d \zeta .
$$

One easily checks that if $h \in H_{0}^{\frac{s}{3}}\left(R^{+}\right)$, then $f \in L^{2}(R)$. Thus, applying Lemma 3.7 with $\theta=1$ and $\beta=1 / 2$ to $\mathcal{U}(t) f(x)$ yields

$$
\left(\int_{0}^{\infty} \sup _{x \in R^{+}}\left|\mathcal{D}_{x}^{1 / 4} \mathcal{U}(t) f(x)\right|^{4} d t\right)^{1 / 4} \leq C\|f\|_{L^{2}(R)} \leq C\|h\|_{H^{\frac{s}{3}}\left(R^{+}\right)}
$$

which gives the inequality required in the lemma using (3.12). The proof is complete.

Attention is now turned to the semigroup $W_{c}(t)$ defined in Proposition 2.1. As mentioned earlier, the corresponding estimates for $W_{c}(t)$, which are similar to those in Lemmas 3.3 to 3.8 , may be obtained directly from the integral representation appearing in Proposition 2.1. However, as pointed out by a referee, there is a short-cut based on the next observation.

Let a function $\phi$ be defined on the half line $R^{+}$and let $\phi^{*}$ be an extension of $\phi$ to the whole line $R$. The mapping $\phi \rightarrow \phi^{*}$ can be organized so that it defines a bounded linear operator from $H^{s}\left(R^{+}\right)$to $H^{s}(R)$ for all $s \geq 0$ (see [47]). Henceforth, $\phi^{*}$ will refer to the result of such an extension operator applied to $\phi \in H^{s}\left(R^{+}\right)$. Assume that $v=v(x, t)$ is the solution of

$$
v_{t}+v_{x}+v_{x x x}=0, \quad v(x, 0)=\phi^{*}(x)
$$

for $x \in R, t \geq 0$. If $g(t)=v(0, t)$, then $v_{g}=v_{g}(x, t)=W_{b}(t) g$ is the corresponding solution of the non-homogeneous boundary-value problem (2.9) with boundary condition $h(t)=g(t)$ for $t \geq 0$. It is clear that for $x>0$ the function $v(x, t)-v_{g}(x, t)$ solves the IBVP (2.1), and this in turn leads to a representation of the semigroup 
$W_{c}(t)$ in terms of $W_{b}(t)$ and $W_{R}(t)$, where $W_{R}(t)$ is the $C_{0}$-semigroup in the space $L^{2}(R)$ generated by the operator $A^{*}$ defined by

$$
A^{*} f=-f^{\prime}-f^{\prime \prime \prime}
$$

with domain $\mathcal{D}\left(A^{*}\right)=H^{3}(R)$ and $v(x, t)=W_{R}(t) \phi^{*}(x)$.

Proposition 3.9. For a given $s \geq 0$ and any $\phi \in H^{s}\left(R^{+}\right)$with $\phi(0)=0$, if $\phi^{*}$ is its extension to $R$ as described above, then $W_{c}(t) \phi$ may be written in the form

$$
W_{c}(t) \phi=W_{R}(t) \phi^{*}-W_{b}(t) g
$$

for any $x, t>0$, where $g$ is the trace of $W_{R}(t) \phi^{*}$ at $x=0$.

To have appropriate estimates of $W_{c}(t) \phi$, the following trace result related to the semigroup $W_{R}(t)$ is needed.

Lemma 3.10. If $s \geq 0$ is given, then there exists a constant $C$ depending only on s such that

$$
\sup _{x \in R}\left\|W_{R}(\cdot) \psi(x)\right\|_{H_{t}^{(s+1) / 3}(R)} \leq C\|\psi\|_{H^{s}(R)}
$$

for all $\psi \in H^{s}(R)$.

Proof. Observe that

$$
\begin{aligned}
W_{R}(t) \psi(x) & =\int_{-\infty}^{+\infty} e^{i\left(\mu^{3}-\mu\right) t} e^{i \mu x} \hat{\psi}(\mu) d \mu \\
& =I_{1}(x, t)+I_{2}(x, t)+I_{3}(x, t)
\end{aligned}
$$

with

$$
I_{1}(x, t)=\int_{\frac{1}{\sqrt{3}}}^{\infty} e^{i\left(\mu^{3}-\mu\right) t} e^{i \mu x} \hat{\psi}(\mu) d \mu, \quad I_{2}(x, t)=\int_{-\frac{1}{\sqrt{3}}}^{\frac{1}{\sqrt{3}}} e^{i\left(\mu^{3}-\mu\right) t} e^{i \mu x} \hat{\psi}(\mu) d \mu,
$$

and

$$
I_{3}(x, t)=\int_{-\infty}^{\frac{1}{\sqrt{3}}} e^{i\left(\mu^{3}-\mu\right) t} e^{i \mu x} \hat{\psi}(\mu) d \mu .
$$

Note that the cubic equation

$$
\eta=\mu^{3}-\mu
$$

has only one real solution $\mu=\delta_{1}(\eta)$ when $1 / \sqrt{3} \leq \mu<\infty$. By a change of variables, we may write

$$
I_{1}(x, t)=\int_{-\frac{2}{3 \sqrt{3}}}^{\infty} e^{i \eta t} e^{i \delta_{1}(\eta) x}\left(3 \delta_{1}^{2}(\eta)-1\right)^{-1} \int_{-\infty}^{\infty} e^{-\delta_{1}(\eta) \xi} \psi(\xi) d \xi d \eta .
$$

Applying the Plancherel Theorem to $I_{1}(x, t)$, there results

$$
\begin{aligned}
\left\|I_{1}(x, \cdot)\right\|_{H^{(s+1) / 3}(R)}^{2} & \leq C \int_{-\frac{2}{3 \sqrt{3}}}^{+\infty}\left|3 \delta_{1}^{2}(\eta)-1\right|^{-2}(1+|\eta|)^{2(s+1) / 3}\left|\hat{\psi}\left(\delta_{1}(\eta)\right)\right|^{2} d \eta \\
& \leq C \int_{\frac{1}{\sqrt{3}}}^{+\infty}(1+\mu)^{2 s}|\hat{\psi}(\mu)|^{2} d \mu=C\|\psi\|_{H^{s}(R)}^{2}
\end{aligned}
$$

for all $x \in R$. 
Similar arguments yield the uniform bounds

$$
\left\|I_{j}(x, \cdot)\right\|_{H_{t}^{(s+1) / 3}(R)} \leq C\|\psi\|_{H^{s}(R)}
$$

for $j=2,3$ and $x \in R$.

The following estimates for $W_{c}(t)$ follow from Proposition 3.9, Lemma 3.10, similar estimates of $W_{R}(t)$ obtained in Kenig, Ponce and Vega [41], and the estimates of $W_{b}(t)$ established earlier in Lemmas 3.3 to 3.8 .

Lemma 3.11. For any given $s \in[0,7 / 2]$, there exists a constant $C$ such that if $\phi \in H_{0}^{s}\left(R^{+}\right)$for $0 \leq s \leq 1$ or $\phi \in H_{0}^{1}\left(R^{+}\right) \cap H^{s}\left(R^{+}\right)$for $s>1$, then

$$
\begin{gathered}
\sup _{0 \leq t<+\infty}\left\|W_{c}(t) \phi\right\|_{H^{s}\left(R^{+}\right)} \leq C\|\phi\|_{H^{s}\left(R^{+}\right)}, \\
\sup _{x \in R^{+}} \int_{0}^{\infty}\left|J_{x}^{s+1} W_{c}(t) \phi(x)\right|^{2} d t \leq C\|\phi\|_{H^{s}\left(R^{+}\right)}^{2}, \\
\sup _{x \in R^{+}}\left\|D_{x}^{k} W_{c}(\cdot) \phi(x)\right\|_{H_{t}^{(s-k+1) / 3}\left(R^{+}\right)} \leq C\|\phi\|_{H^{s}\left(R^{+}\right)}
\end{gathered}
$$

for $k=0,1$, and

$$
\left(\int_{0}^{\infty}\left\|\mathcal{D}^{s+1 / 4} W_{c}(t) \phi\right\|_{L_{x}^{\infty}\left(R^{+}\right)}^{4} d t\right)^{1 / 4} \leq C\|\phi\|_{H^{s}\left(R^{+}\right)} .
$$

In addition, if $s>3 / 4$, then

$$
\left(\int_{0}^{\infty} \sup _{t \in[0, T]}\left|W_{c}(t) \phi(x)\right|^{2} d x\right)^{1 / 2} \leq C(1+T)\|\phi\|_{H^{s}\left(R^{+}\right)}
$$

We conclude this section with a technical lemma which is needed to handle the non-homogeneous boundary condition.

Lemma 3.12. Let $0 \leq s \leq 7 / 2$ and $T>0$ be given. Let $f(x, t)=e^{-x} h(t)$ where $h \in H^{s}\left(R^{+}\right)$. Then there exists a constant $C$ such that the function u given by

$$
u(x, t)=\int_{0}^{t} W_{c}(t-\tau) f(\cdot, \tau) d \tau
$$

obeys the inequalities

$$
\begin{aligned}
& \sup _{0 \leq t<+\infty}\|u(\cdot, t)\|_{H^{s}\left(R^{+}\right)}+\left(\sup _{x \in R^{+}}\left\|J_{x}^{s+1} u(x, t)\right\|_{L_{t}^{2}\left(R^{+}\right)}^{2}\right)^{1 / 2} \\
& +\sum_{k=0}^{1} \sup _{x \in R^{+}}\left\|D_{x}^{k} u\right\|_{H_{t}^{(s-k+1) / 3}\left(R^{+}\right)} \\
& +\left(\int_{0}^{\infty}\left\|\mathcal{D}^{s+1 / 4} u(x, t)\right\|_{L_{x}^{\infty}\left(R^{+}\right)}^{4} d t\right)^{1 / 4}+\left(\int_{0}^{\infty} \sup _{t \in[0, T]}|u(x, t)|^{2} d x\right)^{1 / 2} \\
& \leq \begin{cases}C\|h\|_{L^{2}\left(R^{+}\right)} & \text {for } 0 \leq s \leq 2, \\
C\|h\|_{H^{(s-2) / 3}\left(R^{+}\right)} & \text {for } 2<s \leq 7 / 2 .\end{cases}
\end{aligned}
$$


Proof. Let $\psi(x)$ be an extension of $e^{-x}$ from $R^{+}$to $R$ such that $\psi \in H^{6}(R)$. Then we may write $u(x, t)$ as

$$
u(x, t)=\int_{0}^{t}\left[W_{R}(t-\tau) \psi(\cdot) h(\tau)\right](x) d \tau-\int_{0}^{t}\left[W_{b}(t) \tilde{g}(\cdot-\tau)\right](x) d \tau
$$

where $\tilde{g}(t)$ is the trace of $W_{R}(t) \psi(\cdot) h(\tau)$ at $x=0$. By switching the order of application of the linear operator $W_{b}(t)$ and integration with respect to $\tau$, it appears that $u(x, t)=w(x, t)-\left[W_{b}(t) g\right](x)$ with

$$
w(x, t)=\int_{0}^{t} W_{R}(t-\tau) \psi(\cdot) h(\tau) d \tau
$$

and $g(t)=w(0, t)$. Note that $w(x, t)$ solves

$$
w_{t}(x, t)=-w_{x}(x, t)-w_{x x x}(x, t)+\psi(x) h(t), \quad w(x, 0)=0
$$

for $x, t \in R$. Applying the estimates of the $x$-derivatives of $w(x, t)$ obtained in [41, it is straightforward to see that

$$
\|g(t)\|_{H^{1}\left(R^{+}\right)}=\|w(0, \cdot)\|_{H^{1}\left(R^{+}\right)} \leq C\|h\|_{L^{2}\left(R^{+}\right)}
$$

and

$$
\|g(t)\|_{H^{2}\left(R^{+}\right)}=\|w(0, \cdot)\|_{H^{2}\left(R^{+}\right)} \leq C\|h\|_{H^{1}\left(R^{+}\right)} .
$$

Standard interpolation theory then implies

$$
\|g(t)\|_{H^{1+s}\left(R^{+}\right)}=\|w(0, \cdot)\|_{H^{1+s}\left(R^{+}\right)} \leq C\|h\|_{H^{s}\left(R^{+}\right)}
$$

for $0 \leq s \leq 1$. The classical estimates for $w$ obtained in [41] together with Lemmas 3.3 to 3.8 for $W_{b}(t) g$ yields the inequality in the lemma.

\section{LOCAL WELL-POSEDNESS}

Considered in this section is the fully nonlinear initial-boundary-value problem

$$
\left\{\begin{array}{l}
u_{t}+u_{x}+u u_{x}+u_{x x x}=0, \quad \text { for } x, t \geq 0, \\
u(x, 0)=\phi(x), \quad u(0, t)=h(t) .
\end{array}\right.
$$

for the KdV equation. Solving (4.1) will be shown to define a continuous mapping from the product space $H^{s}\left(R^{+}\right) \times H^{(s+1) / 3}(0, T)$, from which the auxiliary data are drawn, to the space $C\left([0, T] ; H^{s}\left(R^{+}\right)\right)$where the solution $u$ resides if $s>3 / 4$, at least for small values of $T$. This is a result of local well-posedness. While the arguments leading to our result are a little involved, they follow from the estimates put forward in Section 3 together with standard modern ideas for dealing with nonlinear dispersive wave equations.

The development begins with the introduction of several seminorms and some Banach spaces as in the paper of Kenig, Ponce and Vega [41]. For given $s \geq 0$, $T>0$ and any function $w \equiv w(x, t): R^{+} \times[0, T] \rightarrow R$, define

$$
\begin{gathered}
\Lambda_{1, s}^{T}(w) \equiv \sup _{0 \leq t \leq T}\|w(\cdot, t)\|_{H^{s}\left(R^{+}\right)}, \\
\Lambda_{2, s}^{T}(w) \equiv\left(\sup _{x \in R^{+}} \int_{0}^{T}\left|J_{x}^{s+1} w(x, t)\right|^{2} d t\right)^{1 / 2},
\end{gathered}
$$




$$
\begin{gathered}
\Lambda_{3, s}^{T}(w) \equiv \sup _{x \in R^{+}}\|w(x, \cdot)\|_{H^{\frac{s+1}{3}}(0, T)}+\sup _{x \in R^{+}}\left\|D_{x} w(x, \cdot)\right\|_{H^{\frac{s}{3}}(0, T)} \\
\Lambda_{4}^{T}(w) \equiv\left(\int_{0}^{T} \sup _{x \in R^{+}}\left|D_{x} w(x, t)\right|^{4} d t\right)^{1 / 4} \\
\Lambda_{5}^{T}(w)=\left(\int_{0}^{\infty} \sup _{t \in[0, T]}|w(x, t)|^{2} d x\right)^{1 / 2}
\end{gathered}
$$

In addition, let

$$
\lambda_{1, s}^{T}(w)=\max \left\{\Lambda_{1, s}^{T}(w), \Lambda_{2, s}^{T}(w), \Lambda_{3, s}^{T}(w)\right\}
$$

and

$$
\lambda_{T, s}(w)=\lambda_{1, s}^{T}(w)+\Lambda_{4}^{T}(w)+\Lambda_{5}^{T}(w) .
$$

It is convenient to summarize the linear estimates established in Section 3 in terms of these quantities. This reinterpretation is stated as a set of four lemmas.

Lemma 4.1. For a given $s \in[0,7 / 2]$ and $T>0$, there exists a constant $C$ depending only on s such that

$$
\lambda_{1, s}^{T}\left(W_{c}(t) \phi\right) \leq C\|\phi\|_{H^{s}\left(R^{+}\right)}
$$

for $\phi \in H_{0}^{s}\left(R^{+}\right)$if $s \leq 1$ or for $\phi \in H_{0}^{1}\left(R^{+}\right) \cap H^{s}\left(R^{+}\right)$if $s>1$;

$$
\lambda_{1, s}^{T}\left(W_{b}(t) h\right) \leq C\|h\|_{H^{(s+1) / 3}\left(R^{+}\right)}
$$

for $h \in H_{0}^{(s+1) / 3}\left(R^{+}\right)$;

$$
\lambda_{1, s}^{T}\left(\int_{0}^{t} W_{c}(t-\tau) f(x, \tau) d \tau\right) \leq C \int_{0}^{T}\|f(\cdot, \tau)\|_{H^{s}\left(R^{+}\right)} d \tau
$$

for $f \in L^{1}\left(0, T ; H_{0}^{s}\left(R^{+}\right)\right)$if $s \leq 1$ or for $f \in L^{1}\left(0, T ; H_{0}^{1}\left(R^{+}\right) \cap H^{s}\left(R^{+}\right)\right)$if $s>1$.

Lemma 4.2. There exists a constant $C$ such that for any $T>0$,

$$
\Lambda_{4}^{T}\left(W_{c}(t) \phi\right) \leq C\|\phi\|_{H^{1 / 2}\left(R^{+}\right)}
$$

for $\phi \in H_{0}^{1 / 2}\left(R^{+}\right)$;

$$
\Lambda_{4}^{T}\left(W_{b}(t) h\right) \leq C\|h\|_{H^{1 / 2}(0, T)}
$$

for $h \in H_{0}^{1 / 2}\left(R^{+}\right)$;

$$
\Lambda_{4}^{T}\left(\int_{0}^{t} W_{c}(t-\tau) f(x, \tau) d \tau\right) \leq C \int_{0}^{T}\|f(\cdot, \tau)\|_{H^{1 / 2}\left(R^{+}\right)} d \tau
$$

for $f \in L^{1}\left(0, T ; H_{0}^{1 / 2}\left(R^{+}\right)\right)$. 
Lemma 4.3. For any $s>3 / 4$ and $T>0$, there exists a constant $C$ depending only on s such that

$$
\Lambda_{5}^{T}\left(W_{c}(t) \phi\right) \leq C(1+T)\|\phi\|_{H^{s}\left(R^{+}\right)}
$$

for any $\phi \in H^{s}\left(R^{+}\right)$with $\phi(0)=0$;

$$
\Lambda_{5}^{T}\left(W_{b}(t) h\right) \leq C(1+T)\|h\|_{H^{(s+1) / 3}\left(R^{+}\right)}
$$

for any $h \in H^{(s+1) / 3}\left(R^{+}\right)$with $h(0)=0$;

$$
\Lambda_{5}^{T}\left(\int_{0}^{t} W_{c}(t-\tau) f(x, \tau) d \tau\right) \leq C(1+T) \int_{0}^{T}\|f(\cdot, \tau)\|_{H^{s}\left(R^{+}\right)} d \tau
$$

for any $f \in L^{1}\left(0, T ; H^{s}\left(R^{+}\right)\right)$with $f(0, t) \equiv 0$ for $0 \leq t \leq T$.

Remark 4.1. In the above inequalities, the condition $s>3 / 4$ is sharp in the sense that the estimate fails if $s<3 / 4$ (cf. [41]).

Lemma 4.4. Let $f(x, t) \equiv e^{-x} h(t)$, and let $T>0$ be given. For any $s \in[0,7 / 2]$ and $\epsilon>0$, there exists a constant $C$ depending only on $s$ such that if $0 \leq s \leq 2$, then

$$
\lambda_{T, s}\left(\int_{0}^{t} W_{c}(t-\tau) f(x, \tau) d \tau\right) \leq C\left(1+T^{1 / 2}\right)\|h\|_{L^{2}(0, T)}
$$

for any $h \in L^{2}((0, T)$; if $2<s<7 / 2$, then

$$
\lambda_{T, s}\left(\int_{0}^{t} W_{c}(t-\tau) f(x, \tau) d \tau\right) \leq C\left(T^{1 / 2}\|h\|_{L^{2}(0, T)}+\left(1+T^{1 / 2}\right)\|h\|_{H^{\frac{s-2}{3}}(0, T)}\right)
$$

for $h \in H^{\frac{s-2}{3}}(0, T)$.

Consider the initial-boundary-value problem

$$
\begin{cases}u_{t}+u_{x}+u_{x x x}=f, & \text { for } x, t \geq 0, \\ u(x, 0)=\phi(x), & u(0, t)=h(t) .\end{cases}
$$

The preceding lemmas imply the following estimates for its solution $u$.

Proposition 4.5. Let $s \geq 0$ and $\epsilon>0$ be given. There exists a constant $C$ depending only on $s$ and on $\epsilon$ when it appears, such that for any $T>0$,

(i) for $0 \leq s \leq 1 / 2$,

$$
\lambda_{1, s}^{T}(u) \leq C\left(\|\phi\|_{H^{s}\left(R^{+}\right)}+\|h\|_{H^{(s+1) / 3}\left(R^{+}\right)}+\int_{0}^{T}\|f(\cdot, t)\|_{H^{s}\left(R^{+}\right)} d t\right) ;
$$

(ii) for $1 / 2<s \leq 2$ and $\phi(0)=h(0)$,

$$
\begin{aligned}
\lambda_{1, s}^{T}(u) & \leq C\left(\|\phi\|_{H^{s}\left(R^{+}\right)}+\|h\|_{H^{(s+1) / 3}\left(R^{+}\right)}+\int_{0}^{T}\|f(\cdot, t)\|_{H^{s}\left(R^{+}\right)} d t\right) \\
& +C\left(1+T^{1 / 2}\right)\|f(0, \cdot)\|_{L^{2}(0, T)}
\end{aligned}
$$


(iii) for $2 \leq s \leq 3$ and $\phi(0)=h(0)$,

$$
\begin{aligned}
& \lambda_{1, s}^{T}(u) \leq C\left(\|\phi\|_{H^{s}\left(R^{+}\right)}+\|h\|_{H^{(s+1) / 3}\left(R^{+}\right)}+\int_{0}^{T}\|f(\cdot, t)\|_{H^{s}\left(R^{+}\right)} d t\right) \\
& +C\left(1+T^{1 / 2}\right)\|f(0, \cdot)\|_{H_{t}^{\frac{s-2}{3}}(0, T)} ;
\end{aligned}
$$

(iv) if $\phi(0)=h(0)$, then

$$
\begin{aligned}
\Lambda_{4}^{T}(u) \leq & \left.C \quad\|\phi\|_{H^{1 / 2}\left(R^{+}\right)}+\|h\|_{H^{1 / 2}\left(R^{+}\right)}+\int_{0}^{T}\|f(\cdot, t)\|_{H^{1 / 2}\left(R^{+}\right)} d t\right) \\
& +C\left(|\phi(0)|+T^{1 / 4}\|f(0, \cdot)\|_{L^{2}(0, T)}\right)
\end{aligned}
$$

(v) if $\phi(0)=h(0)$, then

$$
\begin{aligned}
\Lambda_{5}^{T}(u) & \leq C\left(\|\phi\|_{H^{\frac{3}{4}+\epsilon}\left(R^{+}\right)}+\|h\|_{H^{\frac{7}{12}}+\epsilon\left(R^{+}\right)}+\int_{0}^{T}\|f(\cdot, t)\|_{H^{\frac{3}{4}+\epsilon}\left(R^{+}\right)} d t\right) \\
& +C T^{1 / 2}\|f(0, \cdot)\|_{L^{2}(0, T)} .
\end{aligned}
$$

Proof. For $0 \leq s \leq 1 / 2$, the solution $u$ of (4.2) is given by

$$
u(x, t)=W_{c}(t) \phi(x)+\left[W_{b}(t) h\right](x)+\int_{0}^{t} W_{c}(t-\tau) f(x, \tau) d \tau,
$$

whereas for $1 / 2<s \leq 3$, by Proposition 2.3, if $\phi(0)=h(0)$, then its solution $u$ can be written as

$$
\begin{gathered}
u(x, t)=W_{c}(t) \phi_{1}(x)+\int_{0}^{t} W_{c}(t-\tau)\left(f_{1}(x, \tau)+e^{-x} f(0, \tau)+2 e^{-x-\tau} h(0)\right) d \tau \\
+\left[W_{b}(t) h_{1}\right](x)+e^{-x-t} h(0)
\end{gathered}
$$

with

$$
\phi_{1}(x)=\phi(x)-e^{-x} \phi(0), \quad f_{1}(x, t)=f(x, t)-e^{-x} f(0, t)
$$

and

$$
h_{1}(t)=h(t)-e^{-t} h(0) .
$$

The advertised estimates then follow by combining the estimates in Lemmas 4.14.4 .

For any $T>0$ and $s$ in the interval $0 \leq s \leq 3$, let $Z_{T}^{s}$ be the collection of all functions $u \in C\left([0, T] ; H^{s}\left(R^{+}\right)\right)$satisfying

$$
\begin{cases}\lambda_{1, s}^{T}(u)<\infty & \text { if } 0 \leq s \leq 1 / 2 ; \\ \lambda_{1, s}^{T}(u)+\Lambda_{4}^{T}(u)<\infty & \text { if } 1 / 2<s \leq 3 / 4 \\ \lambda_{T, s}(u)<\infty & \text { if } 3 / 4<s \leq 3 .\end{cases}
$$


For $v \in Z_{T}^{s}$, define its norm $\|v\|_{Z_{T}^{s}}$ as

$$
\|v\|_{Z_{T}^{s}}= \begin{cases}\lambda_{1, s}^{T}(v), & \text { if } 0 \leq s \leq 1 / 2 \\ \lambda_{1, s}^{T}(v)+\Lambda_{4}^{T}(v), & \text { if } 1 / 2<s \leq 3 / 4 \\ \lambda_{T, s}(v), & \text { if } 3 / 4<s \leq 3\end{cases}
$$

The space $Z_{T}^{s}$ possesses the following property which is one of the keys to establishing the well-posedness of the initial-boundary-value problem under consideration.

Lemma 4.6. Let $3 / 4<s \leq 3$ and $T>0$ be given. For any $u, v \in Z_{T}^{s}$, $u v_{x} \in$ $L^{2}\left(0, T ; H^{s}\left(R^{+}\right)\right)$and

$$
\left\|u v_{x}\right\|_{L^{2}\left(0, T ; H^{s}\left(R^{+}\right)\right)} \leq C\|u\|_{Z_{T}^{s}}\|v\|_{Z_{T}^{s}}
$$

where $C$ depends on $s$, but is independent of $T, u$ and $v$.

Proof. We prove (4.4) for $3 / 4<s<1$. The proof for other values of $s$ is similar. Since

$$
\begin{aligned}
\left\|u v_{x}\right\|_{L^{2}\left(0, T ; H^{s}\left(R^{+}\right)\right)}^{2} & \\
= & \int_{0}^{T}\left\|u(\cdot, t) v_{x}(\cdot, t)\right\|_{L^{2}\left(R^{+}\right)}^{2} d t \\
& +\int_{0}^{T}\left\|J_{x}^{s}\left(u(\cdot, t) v_{x}(\cdot, t)\right)\right\|_{L^{2}\left(R^{+}\right)}^{2} d t
\end{aligned}
$$

and it is straightforward to deduce that

$$
\int_{0}^{T}\left\|u(\cdot, t) v_{x}(\cdot, t)\right\|_{L^{2}\left(R^{+}\right)}^{2} d t \leq C\|u\|_{Z_{T}^{s}}^{2}\|v\|_{Z_{T}^{s}}^{2}
$$

it is only necessary to show that

$$
\int_{0}^{T}\left\|J_{x}^{s}\left(u(\cdot, t) v_{x}(\cdot, t)\right)\right\|_{L^{2}\left(R^{+}\right)}^{2} d t \leq C\|u\|_{Z_{T}^{s}}^{2}\|v\|_{Z_{T}^{s}}^{2}
$$


To this end, argue as follows:

$$
\begin{gathered}
\int_{0}^{T}\left\|J_{x}^{s}\left(u(\cdot, t) v_{x}(\cdot, t)\right)\right\|_{L^{2}\left(R^{+}\right)}^{2} d t \\
=\int_{0}^{T} \int_{0}^{\infty} \tau^{-(2 s+1)} \int_{0}^{\infty}\left|u(x+\tau, t) v_{x}(x+\tau, t)-u(x, t) v_{x}(x, t)\right|^{2} d x d \tau d t \\
\leq 2 \int_{0}^{T} \int_{0}^{\infty} \tau^{-(2 s+1)} \int_{0}^{\infty}|u(x+\tau, t)-u(x, t)|^{2}\left|v_{x}(x+\tau, t)\right|^{2} d x d \tau d t \\
\quad+2 \int_{0}^{T} \int_{0}^{\infty} \tau^{-(2 s+1)} \int_{0}^{\infty}\left|v_{x}(x+\tau, t)-v_{x}(x, t)\right|^{2}|u(x, t)|^{2} d x d \tau d t \\
\leq 2 \int_{0}^{\infty} \int_{0}^{T} \int_{0}^{\infty} \tau^{-(2 s+1)}\left|v_{x}(x+\tau, t)-v_{x}(x, t)\right|^{2}|u(x, t)|^{2} d \tau d t d x \\
+2 \int_{0}^{T}\left\|v_{x}(\cdot, t)\right\|_{L^{\infty}\left(R^{+}\right)}^{2} \int_{0}^{\infty} \tau^{-(2 s+1)} \int_{0}^{\infty}|u(x+\tau, t)-u(x, t)|^{2} d x d \tau d t \\
\leq 2 \int_{0}^{\infty} \sup _{0 \leq t \leq T}|u(x, t)|^{2} \int_{0}^{T} \int_{0}^{\infty} \tau^{-(2 s+1)}\left|v_{x}(x+\tau, t)-v_{x}(x, t)\right|^{2} d \tau d t d x \\
+2 \int_{0}^{T}\left\|v_{x}(\cdot, t)\right\|_{L^{\infty}\left(R^{+}\right)}^{2} \int_{0}^{\infty} \tau^{-(2 s+1)} \int_{0}^{\infty}|u(x+\tau, t)-u(x, t)|^{2} d x d \tau d t \\
\leq \quad 2 \int_{0}^{\infty} \sup _{0 \leq t \leq T}|u(x, t)|^{2} d x \sup _{x \in R^{+}} \int_{0}^{T} \int_{0}^{\infty} \tau^{-(2 s+1)}\left|v_{x}(x+\tau, t)-v_{x}(x, t)\right|^{2} d \tau d t \\
+2 \int_{0}^{T}\left\|v_{x}(\cdot, t)\right\|_{L^{\infty}\left(R^{+}\right)}^{2} \int_{0}^{\infty} \tau^{-(2 s+1)} \int_{0}^{\infty}|u(x+\tau, t)-u(x, t)|^{2} d x d \tau d t \\
\leq \quad\|u\|_{Z_{T}^{s}}^{2}\|v\|_{Z_{T}^{s} \cdot}^{2} .
\end{gathered}
$$

The proof is complete.

Lemma 4.7. Let $s \in[0,3 / 4]$ and $T>0$ be given. Then for any $u \in Z_{T}^{1}$ and $v \in Z_{T}^{s}, u v_{x} \in L^{2}\left(0, T ; H^{s}\left(R^{+}\right)\right)$and

$$
\left\|(u v)_{x}\right\|_{L^{2}\left(0, T ; H^{s}\left(R^{+}\right)\right)} \leq C\|u\|_{Z_{T}^{1}}\|v\|_{Z_{T}^{s}}
$$

where $C$ is independent of $u$ and $v$.

Proof. The proof is similar to the proof of Lemma 4.6.

As in many initial-boundary-value problems, some compatibility conditions are needed for relating the initial data $\phi$ and the boundary value $h$. A simple computation shows that if $u$ is a $C^{\infty}$-smooth solution of (4.1) up to the boundary, then its initial data $u(x, 0)=\phi(x)$ and its boundary value $u(0, t)=h(t)$ must satisfy

$$
\phi_{k}(0)=h_{k}(0)
$$


for $k=0,1, \cdots$, where $h_{k}(t) \equiv h^{(k)}(t)$ is the $k$-th order derivative of $h$,

$$
\left\{\begin{array}{l}
\phi_{0}(x)=\phi(x), \quad \text { and } \\
\phi_{k}(x)=-\left(\phi_{k-1}^{\prime \prime \prime}(x)+\phi_{k-1}^{\prime}(x)+\sum_{j=0}^{k-1}\left[\phi_{j}(x) \phi_{k-j-1}(x)\right]^{\prime}\right)
\end{array}\right.
$$

for $k=1,2, \cdots$.

Definition (s-compatibility). Given $T>0$ and $s \geq 0$, we say that $(\phi, h) \in$ $H^{s}\left(R^{+}\right) \times H^{(s+1) / 3}(0, T)$ is $s$-compatible if

$$
\phi_{k}(0)=h_{k}(0)
$$

for $k=0,1, \cdots,[s / 3]-1$ when $s-3[s / 3] \leq 1 / 2$ and for $k=0,1, \cdots,[s / 3]$ when $s-3[s / 3]>1 / 2$.

Here is the local well-posedness result for the problem (4.1) that is the ultimate focus in this section.

Theorem 4.8. Let $T>0$ and $s \in(3 / 4,3]$ be given. For a pair of s-compatible functions $\phi \in H^{s}\left(R^{+}\right)$and $h \in H^{(1+s) / 3}(0, T)$, there exists a $T^{*} \in(0, T]$ depending only on $\|\phi\|_{H^{s}\left(R^{+}\right)}+\|h\|_{H^{(s+1) / 3(0, T)}}$ such that the problem (4.1) admits a unique solution $u \in Z_{T^{*}}^{s}$.

Remark 4.2. The proof given below shows that the solution map

$$
(\phi, h) \mapsto u
$$

from $H^{s}\left(R^{+}\right) \times H^{(s+1) / 3}(0, T) \rightarrow Z_{T *}^{s}$ is Lipschitz-continuous. It will be shown later in Section 6 that this map has much stronger regularity; namely, it is real analytic.

Proof. For the given $s$-compatible pair $(\phi, h)$, let $0<\beta \leq T$ and $r>0$ be two constants (to be determined later) and define

$$
S_{\beta, r}=\left\{w \in Z_{\beta}^{s}: \quad w(0, t)=h(t), \quad w(x, 0)=\phi(x), \quad\|w\|_{Z_{\beta}^{s}} \leq r\right\} .
$$

The set $S_{\beta, r}$ is a closed subset of the space $Z_{\beta}^{s}$. According to Proposition 4.5, for any $v \in S_{\beta, s}$, the linear problem

$$
\left\{\begin{array}{l}
u_{t}+u_{x}+u_{x x x}=-v v_{x}, \quad \text { for } x, t \geq 0, \\
u(x, 0)=\phi(x), \quad u(0, t)=h(t)
\end{array}\right.
$$

has a unique solution $u \in Z_{\beta}^{s}$. Thus (4.8) defines a map $\Gamma$ from $Z_{\beta}^{s}$ to $Z_{\beta}^{s}$, say

$$
u=\Gamma(v)
$$

for any $v \in Z_{\beta}^{s}$. In addition, for any $\epsilon>0$, there exists a constant $C$ such that

$$
\begin{aligned}
& \lambda_{\beta, s}(\Gamma(v)) \leq C\left(\|\phi\|_{H^{s}\left(R^{+}\right)}+\|h\|_{H^{(s+1) / 3}(0, T)}\right) \\
& +C\left(\int_{0}^{\beta}\left\|v(\cdot, \tau) v_{x}(\cdot, \tau)\right\|_{H^{s}\left(R^{+}\right)} d \tau+\left\|v(0, \cdot) v_{x}(0, \cdot)\right\|_{L^{2}(0, \beta)}\right)
\end{aligned}
$$


if $3 / 4<s \leq 2$ and

$$
\begin{aligned}
& \lambda_{\beta, s}(\Gamma(v)) \leq C\left(\int_{0}^{\beta}\left\|v(\cdot, \tau) v_{x}(\cdot, \tau)\right\|_{H^{s}\left(R^{+}\right)} d \tau+\|\phi\|_{H^{s}\left(R^{+}\right)}\right) \\
& +C\left(\left(\beta^{1 / 2}+\beta^{1 / 4}\right)\left\|v(0, \cdot) v_{x}(0, \cdot)\right\|_{H^{\frac{s-2}{3}}(0, \beta)}+\|h\|_{H^{\frac{s+1}{3}}(0, T)}\right)
\end{aligned}
$$

if $2<s \leq 3$. By Lemma 4.6 , it is known that

$$
\int_{0}^{\beta}\left\|v(\cdot, \tau) v_{x}(\cdot, \tau)\right\|_{H^{s}\left(R^{+}\right)} d \tau \leq C \beta^{1 / 2} \lambda_{\beta, s}(v) \lambda_{\beta, s}(v) .
$$

In addition, for $3 / 4<s \leq 2$, it is clear that

$$
\begin{aligned}
\left(\int_{0}^{\beta}\left|v_{x}(0, t) v(0, t)\right|^{2} d t\right)^{1 / 2} & =\left(\int_{0}^{\beta}\left|v_{x}(0, t) h(t)\right|^{2} d t\right)^{1 / 2} \\
& \leq C\left(\int_{0}^{\beta}\left|v_{x}(0, t)\right|^{2} d t\right)^{1 / 2}\|h\|_{H^{(s+1) / 3}(0, T)} \\
& \leq C \beta^{1 / 4} \lambda_{T, s}(v)\|h\|_{H^{(s+1) / 3}(0, T)} .
\end{aligned}
$$

If $2<s \leq 3$, then

$$
\begin{aligned}
\left\|v_{x}(0, \cdot) v(0, \cdot)\right\|_{H^{(s-2) / 3}(0, \beta)} & \leq C \beta^{\frac{5-s}{6}}\left\|v_{x}(0, \cdot) v(0, \cdot)\right\|_{H^{(s+4) / 9}(0, \beta)} \\
& \leq C \beta^{\frac{5-s}{6}} \lambda_{\beta, s}(v) \lambda_{\beta, s}(v) .
\end{aligned}
$$

Combining the above estimates yields

$$
\lambda_{\beta, s}(\Gamma(v)) \leq C\left(\|\phi\|_{H^{s}\left(R^{+}\right)}+\|h\|_{H^{(1+s) / 3}(0, T)}+\left(\beta^{1 / 4}+\beta^{1 / 2}\right) \lambda_{\beta, s}^{2}(v)\right)
$$

for any $s \in(3 / 4,3]$ and $0<\beta \leq T$. Here $C$ is independent of $\phi, h$ and $\beta$. Setting

$$
r=2 C\left(\|\phi\|_{H^{s}\left(R^{+}\right)}+\|h\|_{H^{(1+s) / 3}(0, T)}\right)
$$

and choosing $\beta \in(0, T]$ such that

$$
C\left(\beta^{1 / 4}+\beta^{1 / 2}\right) r \leq 1 / 2,
$$

it is seen immediately that

$$
\lambda_{\beta, s}(\Gamma(v)) \leq r \quad \text { for any } v \in S_{\beta, r} .
$$

Thus $\Gamma$ is a map from $S_{\beta, r}$ to $S_{\beta, r}$ if $\beta$ and $r$ are chosen according to (4.9) and (4.10). A similar argument shows that for such $\beta$ and $r$,

$$
\lambda_{\beta, s}\left(\Gamma\left(v_{1}\right)-\Gamma\left(v_{2}\right)\right) \leq \frac{1}{2} \lambda_{\beta, s}\left(v_{1}-v_{2}\right)
$$

for any $v_{1}, v_{2} \in S_{\beta, r}$. Thus $\Gamma$ is a contraction from $S_{\beta, r}$ to $S_{\beta, r}$. Its unique fixed point is the desired solution of (4.1); it is defined on the temporal interval $[0, \beta]$.

The next step is to extend Theorem 4.8 to the case where $s>3$. First, the definition of the space $Z_{T}^{s}$ is extended to values of $s>3$. For $s>3$, write $s$ in the form

$$
s=3 m+s^{\prime}
$$


with $m=[s / 3]$ or $m=[s / 3]-1$ and $0<s^{\prime} \leq 3$. For a given $T>0$ and such a value $s$, let $Z_{T}^{s}$ be the collection of all functions $u \in C^{m-1}\left([0, T] ; H^{3}\left(R^{+}\right)\right)$with $\partial_{t}^{m} u \in C\left([0, T] ; H^{s^{\prime}}\left(R^{+}\right)\right)$satisfying

$$
\|u\|_{Z_{T}^{s}}=\left\|\partial_{t}^{m} u\right\|_{Z_{T}^{s^{\prime}}}+\sum_{k=0}^{m-1}\left\|\partial_{t}^{k} u\right\|_{Z_{T}^{3}}<+\infty .
$$

Theorem 4.9. Let $T>0$ and $s>3$ be given with $s=3 m+s^{\prime}$ where $m=$ $[s / 3]$ and $0<s^{\prime} \leq 3$. For any given pair of $s$-compatible functions $(\phi, h) \in$ $H^{s}\left(R^{+}\right) \times H^{(s+1) / 3}(0, T)$, there exists a $T^{*} \in(0, T]$ depending only on $\|\phi\|_{H^{s}\left(R^{+}\right)}+$ $\|h\|_{H^{(s+1)}(0, T)}$ such that 4.1 admits a unique solution $u \in Z_{T^{*}}^{s}$.

Proof. As in the proof of Theorem 4.8, for the given $s$-compatible $(\phi, h) \in H^{s}\left(R^{+}\right) \times$ $H^{(s+1) / 3}(0, T)$, let $\beta \in(0, T]$ and $r>0$ be two constants to be determined and let

$$
S_{\beta, r}=\left\{w \in Z_{\beta}^{s}: w(0, t)=h(t), w(x, 0)=\phi(x),\|w\|_{Z_{\beta}^{s}} \leq r\right\} .
$$

The set $S_{\beta, r}$ is a closed subspace of $Z_{\beta}^{s}$. Define the map $\Gamma$ from $Z_{\beta}^{s}$ to $Z_{\beta}^{s}$ by

$$
u=\Gamma(v)
$$

where $v \in Z_{\beta, s}$ and $u$ is the unique solution of the linear problem

$$
\left\{\begin{array}{l}
u_{t}+u_{x}+u_{x x x}=-v v_{x}, \quad \text { for } x, t \geq 0, \\
u(x, 0)=\phi(x), \quad u(0, t)=h(t) .
\end{array}\right.
$$

As above, it will transpire that if $\beta$ and $r$ are appropriately chosen, then $\Gamma$ is a contraction map from $S_{\beta, r}$ to $S_{\beta, r}$.

The proof of Theorem 4.8 implies that there is a constant $C$ such that

$$
\lambda_{\beta, 3}(\Gamma(v)) \leq C\left(\|(\phi, h)\|_{H^{s}\left(R^{+}\right) \times H^{(s+1) / 3}(0, T)}+\left(\beta^{1 / 2}+\beta^{1 / 4}\right) \lambda_{\beta, 3}^{2}(v)\right) .
$$

Let $w^{(k)}=\partial_{t}^{k} \Gamma(v)$ for $k=1,2, \cdots, m$. The function $w^{(k)}$ solves the initialboundary-value problem

$$
\left\{\begin{array}{l}
w_{t}^{(k)}+w_{x}^{(k)}+w_{x x x}^{(k)}=-\left(\partial_{t}^{k}\left(v v_{x}\right), \quad \text { for } x, t \geq 0\right. \\
w^{(k)}(x, 0)=\phi_{k}(x), \quad w^{(k)}(0, t)=h_{k}(t),
\end{array}\right.
$$

for $k=1,2, \cdots, m$. By Proposition 4.5, there is a constant $C$ such that

$$
\lambda_{\beta, 3}\left(w^{(k)}\right) \leq C\left(\left\|\left(\phi_{k}, h_{k}\right)\right\|_{H^{3}\left(R^{+}\right) \times H^{(3+1) / 3}(0, T)}+\left\|\partial_{t}^{k}\left(v v_{x}\right)\right\|_{L^{1}\left(0, \beta ; H^{3}\left(R^{+}\right)\right)}\right)
$$

for $k=1, \cdots, m-1$. In addition, we know that

$$
\begin{array}{r}
\lambda_{1, s^{\prime}}^{\beta}\left(w^{(m)}\right) \leq C\left(\left\|\phi_{m}\right\|_{H^{s^{\prime}}\left(R^{+}\right)}+\left\|h_{m}\right\|_{H^{\left(s^{\prime}+1\right) / 3}(0, T)}\right. \\
\left.+\left\|\partial_{t}^{m}\left(v v_{x}\right)\right\|_{L^{1}\left(0, \beta ; H^{s^{\prime}}\left(R^{+}\right)\right)}\right)
\end{array}
$$

if $0<s^{\prime} \leq 1 / 2$

$$
\begin{array}{r}
\lambda_{1, s^{\prime}}^{\beta}\left(w^{(m)}\right)+\Lambda_{4}^{\beta}\left(w^{(m)}\right) \leq C\left(\left\|\phi_{m}\right\|_{H^{s^{\prime}}\left(R^{+}\right)}+\left\|h_{m}\right\|_{H^{\left(s^{\prime}+1\right) / 3}(0, T)}\right) \\
+C\left(\left\|\partial_{t}^{m}\left(v v_{x}\right)\right\|_{L^{1}\left(0, \beta ; H^{s^{\prime}}\left(R^{+}\right)\right)}+\left\|\left(\partial_{t}^{m}\left(v v_{x}\right)\right)(0, t)\right\|_{L^{2}(0, \beta)}\right)
\end{array}
$$


if $1 / 2<s^{\prime} \leq 3 / 4$;

$$
\begin{aligned}
\lambda_{\beta, s^{\prime}}\left(w^{(m)}\right) & \leq C\left(\left\|\phi_{m}\right\|_{H^{s^{\prime}}\left(R^{+}\right)}+\left\|h_{m}\right\|_{H^{\left(s^{\prime}+1\right) / 3}(0, T)}\right) \\
+ & C\left(\left\|\partial_{t}^{m}\left(v v_{x}\right)\right\|_{L^{1}\left(0, \beta ; H^{s^{\prime}}\left(R^{+}\right)\right)}+\left\|\left(\partial_{t}^{m}\left(v v_{x}\right)\right)(0, t)\right\|_{L^{2}(0, \beta)}\right)
\end{aligned}
$$

if $3 / 4<s^{\prime} \leq 2$; and

$$
\begin{aligned}
& \lambda_{\beta, s^{\prime}}\left(w^{(m)}\right) \leq C\left(\left\|\phi_{m}\right\|_{H^{s^{\prime}}\left(R^{+}\right)}+\left\|h_{m}\right\|_{H^{\left(s^{\prime}+1\right) / 3}(0, T)}\right) \\
& \quad+C\left(\left\|\partial_{t}^{m}\left(v v_{x}\right)\right\|_{L^{1}\left(0, \beta ; H^{s^{\prime}}\left(R^{+}\right)\right)}+\left\|\left(\partial_{t}^{m}\left(v v_{x}\right)\right)(0, t)\right\|_{H^{(s-2) / 3}(0, \beta)}\right)
\end{aligned}
$$

if $2<s^{\prime} \leq 3$. Since $v \in Z_{\beta}^{s}$, by repeated application of Lemma 4.6, there follows the inequality

$$
\begin{aligned}
& \left\|\partial_{t}^{k}\left(v v_{x}\right)\right\|_{L^{1}\left(0, \beta ; H^{\left.s^{\prime}\left(R^{+}\right)\right)}\right.} \leq C \beta^{1 / 2} \sum_{j=0}^{k}\left(\begin{array}{c}
k \\
j
\end{array}\right)\left\|\left(\partial_{t}^{j} v \partial_{t}^{k-j} v\right)_{x}\right\|_{L^{2}\left(0, \beta ; H^{s^{\prime}}\left(R^{+}\right)\right)} \\
& \quad \leq C \beta^{1 / 2} \sum_{j=0}^{k}\left(\begin{array}{c}
k \\
j
\end{array}\right) \lambda_{\beta, 3}\left(\partial_{t}^{j} v\right) \lambda_{\beta, 3}\left(\partial_{t}^{k-j} v\right) \\
& \quad \leq C \beta^{1 / 2}\|v\|_{Z_{\beta}^{s}}^{2}
\end{aligned}
$$

for $k=1, \cdots, m-1$. Similarly, using Lemma 4.6 and Lemma 4.7 , it is seen that

$$
\left\|\partial_{t}^{m}\left(v v_{x}\right)\right\|_{L^{1}\left(0, \beta ; H^{s^{\prime}}\left(R^{+}\right)\right)} \leq C \beta^{1 / 2}\|v\|_{Z_{\beta}^{s}}^{2} .
$$

Furthermore, it is straightforward to show that

$$
\left\|\left(\partial_{t}^{m}\left(v v_{x}\right)\right)(0, t)\right\|_{L^{2}(0, \beta)} \leq C \beta^{1 / 4}\|v\|_{Z_{\beta}^{s}}^{2}
$$

when $1 / 2<s^{\prime} \leq 2$, and that

$$
\left\|\left(\partial_{t}^{m}\left(v v_{x}\right)\right)(0, t)\right\|_{H^{(s-2) / 3}(0, \beta)} \leq C\left(\beta^{1 / 2}+\beta^{1 / 4}\right)\|v\|_{Z_{\beta}^{s}}^{2}
$$

when $2<s^{\prime} \leq 3$.

Those estimates, together with (4.11) yield

$$
\|\Gamma(v)\|_{Z_{\beta}^{s}} \leq C\left(\|(\phi, h)\|_{H^{s}\left(R^{+}\right) \times H^{(s+1) / 3}(0, T)}+\left(\beta^{1 / 2}+\beta^{1 / 4}\right)\|v\|_{Z_{\beta}^{s}}^{2}\right) .
$$

The remainder of the proof is the same as the culmination of the proof for Theorem 4.8. The theorem is thereby proved.

By writing the equation in (4.1) in the form

$$
u_{x x x}=-u_{t}-u_{x}-u u_{x}
$$

one sees that if both $u$ and $u_{t}$ belong to the space $C\left([0, T] ; H^{s}\left(R^{+}\right)\right)$for some $s>1 / 2$, then $u \in C\left([0, T] ; H^{s+3}\left(R^{+}\right)\right)$. Thus the following theorem is a direct consequence of Theorem 4.8 and Theorem 4.9.

Theorem 4.10. Let $T>0$ and $s>3 / 4$ be given. Then for any $s$-compatible pair $(\phi, h) \in H^{s}\left(R^{+}\right) \times H^{(s+1) / 3}(0, T)$, there exists a $T^{*} \in(0, T]$ depending only on 
$\|(\phi, h)\|_{H^{s}\left(R^{+}\right) \times H^{(s+1) / 3}(0, T)}$ such that 4.1$)$ with initial data $\phi$ and boundary data $h$ admits a unique solution

$$
u \in Z_{T^{*}}^{s} \cap C\left(\left[0, T^{*}\right] ; H^{s}\left(R^{+}\right)\right)
$$

with $\partial_{t}^{k} u \in C\left(\left[0, T^{*}\right] ; H^{s-3 k}\left(R^{+}\right)\right)$for $k=0,1, \cdots,[s / 3]$.

\section{Global Well-POSEDNESS}

The well-posedness results presented in Section 4 are local in the sense that the length of the time interval $\left[0, T^{*}\right]$ on which the solution exists depends on the quantity $\|\phi\|_{H^{s}\left(R^{+}\right)}+\|h\|_{H^{(s+1) / 3}(0, T)}$. In general, the larger is $\|\phi\|_{H^{s}\left(R^{+}\right)}+$ $\|h\|_{H^{(s+1) / 3}(0, T)}$, the smaller will be $T^{*}$. However, if $T^{*}=T$ no matter what the size of $\|\phi\|_{H^{s}\left(R^{+}\right)}+\|h\|_{H^{(s+1) / 3(0, T)}}$, the initial-boundary-value problem (4.1) is said to be globally well-posed. With local well-posedness in hand, it is well understood that one need only establish a priori global $H^{s}\left(R^{+}\right)$-estimates for the smooth solution $u$ of (4.1) to show that 4.1) is globally well-posed.

In this section, aided by the smoothing properties established in Section 3, a range of a priori estimates is provided and these are established under the same hypotheses as those used to prove the local well-posedness when $s \geq 3$, while a slightly stronger assumption on the boundary data $h$ is employed when $1 \leq s<3$. The theory begins with $H^{s}\left(R^{+}\right)$-bounds in the range $1 \leq s \leq 3$.

Theorem 5.1. Let $T>0$ and $s \in[1,3]$ be given. Then there exists a continuous non-decreasing function $\alpha_{s}: R^{+} \rightarrow R^{+}$such that for any smooth solution $u$ of (4.1),

$$
\sup _{0 \leq t \leq T}\|u(\cdot, t)\|_{H^{s}\left(R^{+}\right)} \leq \alpha_{s}\left(\|\phi\|_{H^{s}\left(R^{+}\right)}+\|h\|_{H^{\frac{7+3 s}{12}}(0, T)}\right) .
$$

Two important tools will be utilized in the proof of this theorem. One is the smoothing properties of the equation established earlier. These will be used to recover the regularity lost through taking boundary traces. The other is nonlinear interpolation theory as expounded in Tartar [57] and Bona and Scott 8], which is the key to obtaining the estimate (5.1) for $1<s<3$.

Here is a précis of the (real) interpolation theory as it will be used below. Let $B_{0}$ and $B_{1}$ be two Banach spaces such that $B_{1} \subset B_{0}$ with continuous inclusion map. Let $f \in B_{0}$ and, for $t \geq 0$, define

$$
K(f, t)=\inf _{g \in B_{1}}\left\{\|f-g\|_{B_{0}}+t\|g\|_{B_{1}}\right\} .
$$

For $0<\theta<1$ and $1 \leq p \leq+\infty$, define

$$
\left[B_{0}, B_{1}\right]_{\theta, p}=B_{\theta, p}=\left\{f \in B_{0}:\|f\|_{\theta, p}=\left(\int_{0}^{\infty} K(f, t)^{p} t^{-\theta p-1} d t\right)^{1 / p}<+\infty\right\}
$$

with the usual modification for the case $p=+\infty$. Then $B_{\theta, p}$ is a Banach space with norm $\|\cdot\|_{\theta, p}$. Given two pairs of indices $\left(\theta_{1}, p_{1}\right)$ and $\left(\theta_{2}, p_{2}\right)$ as above, then $\left(\theta_{1}, p_{1}\right)<\left(\theta_{2}, p_{2}\right)$ means

$$
\begin{cases}\theta_{1}<\theta_{2}, & \text { or } \\ \theta_{1}=\theta_{2} & \text { and } p_{1}>p_{2} .\end{cases}
$$

If $\left(\theta_{1}, p_{1}\right)<\left(\theta_{2}, p_{2}\right)$, then $B_{\theta_{2}, p_{2}} \subset B_{\theta_{1}, p_{1}}$ and the inclusion map is continuous. 
Theorem 5.2. Let $B_{0}^{j}$ and $B_{1}^{j}$ be Banach spaces such that $B_{1}^{j} \subset B_{0}^{j}$ with continuous inclusion mappings, $j=1,2$. Let $\lambda$ and $q$ lie in the ranges $0<\lambda<1$ and $1 \leq q \leq+\infty$. Suppose $A$ is a mapping such that

i) $A: B_{\lambda, q}^{1} \rightarrow B_{0}^{2}$ and for $f, g \in B_{\lambda, q}^{1}$,

$$
\|A f-A g\|_{B_{0}^{2}} \leq C_{0}\left(\|f\|_{B_{\lambda, q}^{1}}+\|g\|_{B_{\lambda, q}^{1}}\right)\|f-g\|_{B_{0}^{1}}
$$

and

ii) $A: B_{1}^{1} \rightarrow B_{1}^{2}$ and for $h \in B_{1}^{1}$

$$
\|A h\|_{B_{1}^{2}} \leq C_{1}\left(\|h\|_{B_{\lambda, q}^{1}}\right)\|h\|_{B_{1}^{1}}
$$

where $C_{j}: R^{+} \rightarrow R^{+}$are continuous non-decreasing functions, $j=0,1$.

Then if $(\theta, p) \geq(\lambda, q)$, A maps $B_{\theta, p}^{1}$ into $B_{\theta, p}^{2}$ and for $f \in B_{\theta, p}^{1}$

$$
\|A f\|_{B_{\theta, p}^{2}} \leq C\left(\|f\|_{B_{\lambda, q}^{1}}\right)\|f\|_{B_{\theta, p}^{1}},
$$

where for $r>0, C(r)=4 C_{0}(4 r)^{1-\theta} C_{1}(3 r)^{\theta}$.

Remark 5.1. This theorem is identical with Theorem 2 of Tartar [57] except that Tartar makes the more restrictive assumption that the constants $C_{0}$ and $C_{1}$ depend only on the $B_{0}^{1}$ norms of the functions in question. Theorem 5.2 was used by Bona and Scott $[8]$ to provide the original proof of global well-posedness of the pure initial-value problem for the $\mathrm{KdV}$ equation on the whole line in fractional order Sobolev spaces $H^{s}(R)$.

Nonlinear interpolation theory as embodied in Theorem 5.2 will be used to prove the estimate (5.1).

Proof of Theorem 5.1. For $T>0$ and $1 \leq s \leq 3$, let

$$
V_{T}^{s}=\left\{(\phi, h) \in H^{s}\left(R^{+}\right) \times H^{\frac{7+3 s}{12}}(0, T) \mid \phi(0)=h(0)\right\}
$$

with the inherited norm from the product space $H^{s}\left(R^{+}\right) \times H^{\frac{7+3 s}{12}}(0, T)$. To apply Theorem 5.2, choose

$$
B_{0}^{1}=V_{T}^{1}, \quad B_{0}^{2}=V_{T}^{3}, \quad B_{1}^{1}=C\left([0, T] ; H^{1}\left(R^{+}\right)\right), \quad B_{1}^{2}=C\left([0, T] ; H^{3}\left(R^{+}\right)\right) .
$$

Let $A$ be the solution map for the IBVP (4.1): $u=A(\phi, h)$. For a given $s$ with $1<s<3$, choose $p=2$ and $\theta=(3-s) / 2$, so that

$$
B_{\theta, p}^{2}=C\left([0, T] ; H^{s}\left(R^{+}\right)\right) \quad \text { and } \quad B_{\theta, p}^{1}=V_{T}^{s} .
$$

The following two propositions are needed to assure both the hypotheses (i) and (ii) in Theorem 5.2 are satisfied in the present context.

Proposition 5.3. For a given $T>0$, there is a $T$-dependent and non-decreasing continuous function $\alpha_{T}: R^{+} \rightarrow R^{+}$such that any smooth solution $u$ of (4.1) satisfies

$$
\sup _{0 \leq t \leq T}\|u(\cdot, t)\|_{H^{1}\left(R^{+}\right)} \leq \alpha_{T}\left(\|(\phi, h)\|_{V_{T}^{1}}\right) .
$$

Proposition 5.4. For a given $T>0$, there is a $T$-dependent and non-decreasing function $\alpha_{T}: R^{+} \rightarrow R^{+}$such that any smooth solution $u$ of 4.1) satisfies

$$
\sup _{0 \leq t \leq T}\|u(\cdot, t)\|_{H^{3}\left(R^{+}\right)} \leq \alpha_{T}\left(\|(\phi, h)\|_{V_{T}^{1}}\right)\|(\phi, h)\|_{V_{T}^{3}} .
$$


If Propositions 5.3 and 5.4 are valid, then hypothesis (ii) is assured by (5.3). To see hypothesis (i) is also satisfied, let $u_{1}$ and $u_{2}$ be two smooth solutions of the equation in (4.1) with

$$
u_{j}(x, 0)=\psi_{j}(x), \quad u_{j}(0, t)=g_{j}(t)
$$

for $j=1,2$ and let $z(x, t)=u_{1}(x, t)-u_{2}(x, t)$. Then $z(x, t)$ solves

$$
\left\{\begin{array}{l}
z_{t}+z_{x}+(a(x, t) z)_{x}+z_{x x x}=0, \quad x>0, \quad t>0, \\
z(x, 0)=\psi_{1}(x)-\psi_{2}(x), \quad z(0, t)=g_{1}(t)-g_{2}(t)
\end{array}\right.
$$

where $a(x, t)=\frac{1}{2}\left(u_{1}(x, t)+u_{2}(x, t)\right)$. It follows from estimate (5.2) and Theorem 4.9 that $a \in Z_{T}^{1}$ and

$$
\|a\|_{Z_{T}^{1}} \leq \alpha_{T}\left(\left\|\left(\psi_{1}, g_{1}\right)\right\|_{V_{T}^{1}}+\left\|\left(\psi_{2}, g_{2}\right)\right\|_{V_{T}^{1}}\right) .
$$

Then, by Proposition 6.1 in the next section, which is proved independently of the present considerations, we have that

$$
\begin{gathered}
\sup _{0 \leq t \leq T}\left\|u_{1}(\cdot, t)-u_{2}(\cdot, t)\right\|_{H^{1}\left(R^{+}\right)} \leq \alpha_{T}\left(\left\|\left(\psi_{1}, g_{1}\right)\right\|_{V_{T}^{1}}+\left\|\left(\psi_{2}, g_{2}\right)\right\|_{V_{T}^{1}}\right) \\
\times\left\|\left(\psi_{1}-\psi_{2}, g_{1}-g_{2}\right)\right\|_{V_{T}^{1}} .
\end{gathered}
$$

Thus Theorem 5.1 follows by a direct application of Theorem 5.2.

Consideration is turned to proving Proposition 5.3 and Proposition 5.4.

Proof of Proposition 5.3. For a smooth solution $u$ of (4.1), write it in the form $u=w+v+g(x, t)$ where $g(x, t) \equiv e^{-x-t} h(0), v$ solves

$$
\left\{\begin{array}{l}
v_{t}+v_{x}+v_{x x x}=0, \quad \text { for } x, t \geq 0, \\
v(x, 0)=0, \quad v(0, t)=h^{*}(t) \equiv h(t)-e^{-t} h(0),
\end{array}\right.
$$

and $w$ solves

$$
w_{t}+w_{x}+w w_{x}+(v w)_{x}+(g w)_{x}+w_{x x x}=Y(x, t)-(g v)_{x}-v v_{x}
$$

with $Y(x, t)=3 g(x, t)+g^{2}(x, t)$ and with the auxiliary conditions

$$
w(x, 0)=\phi^{*}(x) \equiv \phi(x)-e^{-x} \phi(0) \quad \text { and } \quad w(0, t)=0 .
$$

By Proposition 4.5 and the third part of Lemma 3.11, there is a $T$-dependent constant $C_{T}$ such that

$$
\left\{\begin{array}{l}
\|v\|_{Z_{T}^{1}} \leq C_{T}\|h\|_{H^{2 / 3}(0, T)} \text { and } \\
\left(\int_{0}^{T} \sup _{x \in R^{+}}\left|v_{x x}(x, t)\right|^{4} d t\right)^{1 / 4} \leq C_{T}\|h\|_{H^{5 / 6}(0, T)}
\end{array}\right.
$$


for any $(\phi, h) \in V_{T}^{1}$. Multiplying both sides of (15.4) by $2 w$, integrating with respect to $x$ over $(0, \infty)$, and integrating by parts appropriately, there obtains

$$
\begin{aligned}
& \frac{d}{d t} \int_{0}^{\infty} w^{2}(x, t) d x+w_{x}^{2}(0, t)=-\int_{0}^{\infty} v(x, t) v_{x}(x, t) w(x, t) d x \\
& \quad-\int_{0}^{\infty} v_{x}(x, t) w^{2}(x, t) d x \\
& +2 \int_{0}^{\infty}\left(Y(x, t)-(g(x, t) w(x, t))_{x}-(g(x, t) v(x, t))_{x}\right) w(x, t) d x .
\end{aligned}
$$

Hölder's inequality gives

$$
\begin{gathered}
\int_{0}^{t} \int_{0}^{\infty}\left|v_{x}(x, \tau)\right| w^{2}(x, \tau) d x d t \leq \int_{0}^{t} \sup _{x \in R^{+}}\left|v_{x}(x, \tau)\right| \int_{0}^{\infty} w^{2}(x, \tau) d x d \tau \\
\leq\left(\int_{0}^{t} \sup _{x \in R^{+}}\left|v_{x}(x, \tau)\right|^{4} d \tau\right)^{1 / 4}\left(\int_{0}^{t}\|w(\cdot, \tau)\|_{L^{2}\left(R^{+}\right)}^{8 / 3} d \tau\right)^{3 / 4} \\
\leq\|v\|_{Z_{T}^{1}}\left(\int_{0}^{t}\|w(\cdot, \tau)\|_{L^{2}\left(R^{+}\right)}^{8 / 3} d \tau\right)^{3 / 4} \\
\leq\|v\|_{Z_{T}^{1}} \sup _{0 \leq \tau \leq t}\|w(\cdot, \tau)\|_{L^{2}\left(R^{+}\right)}\left(\int_{0}^{t}\|w(\cdot, \tau)\|_{L^{2}\left(R^{+}\right)}^{4 / 3} d \tau\right)^{3 / 4} \\
\leq C_{T}\|v\|_{Z_{T}^{1}} \sup _{0 \leq \tau \leq t}\|w(\cdot, \tau)\|_{L^{2}\left(R^{+}\right)}\left(\int_{0}^{t}\|w(\cdot, \tau)\|_{L^{2}\left(R^{+}\right)}^{2} d \tau\right)^{1 / 2}
\end{gathered}
$$

and

$$
\begin{aligned}
\int_{0}^{t} \int_{0}^{\infty}\left|v(x, \tau) v_{x}(x, \tau) w(x, \tau)\right| d x d \tau & \leq \int_{0}^{t}\|v(\cdot, \tau)\|_{H^{1}\left(R^{+}\right)}^{2}\|w(\cdot, \tau)\|_{L^{2}\left(R^{+}\right)} d \tau \\
& \leq\|v\|_{Z_{T}^{1}}^{2} \int_{0}^{t}\|w(\cdot, \tau)\|_{L^{2}\left(R^{+}\right)} d \tau
\end{aligned}
$$

Similarly, one has

$$
\begin{gathered}
\int_{0}^{t} \int_{0}^{\infty}|g(x, \tau)| w^{2}(x, \tau) d x d \tau \leq\|\phi\|_{H^{1}\left(R^{+}\right)} \int_{0}^{t} \int_{0}^{\infty} w^{2}(x, \tau) d x d \tau \\
\int_{0}^{t} \int_{0}^{\infty}|Y(x, t) w(x, t)| d x d \tau \leq C_{T}\|\phi\|_{H^{1}\left(R^{+}\right)}^{2}\left(\int_{0}^{t} \int_{0}^{\infty} w^{2}(x, \tau) d x d \tau\right)^{1 / 2}
\end{gathered}
$$

and

$$
\begin{gathered}
\int_{0}^{t} \int_{0}^{\infty}\left|(g(x, \tau) v(x, \tau))_{x} w(x, \tau)\right| d x d \tau \\
\leq C_{T}\|\phi\|_{H^{1}\left(R^{+}\right)}\|v\|_{Z_{T}^{1}}\left(\int_{0}^{t} \int_{0}^{\infty} w^{2}(x, \tau) d x d \tau\right)^{1 / 2} .
\end{gathered}
$$


Integrating (5.7) with respect to the temporal variable over $[0, t]$ and combining the above inequalities with (5.6) allows one to infer that

$$
\begin{aligned}
& \int_{0}^{\infty} w^{2}(x, t) d x+\int_{0}^{t} w_{x}^{2}(0, \tau) d \tau \leq \frac{1}{2} \sup _{0 \leq \tau \leq t}\|w(\cdot, \tau)\|_{L^{2}\left(R^{+}\right)}^{2} \\
& \quad+\alpha_{0}+\alpha_{1} \int_{0}^{t}\|w(\cdot, \tau)\|_{L^{2}\left(R^{+}\right)} d \tau+\alpha_{2} \int_{0}^{t}\|w(\cdot, \tau)\|_{L^{2}\left(R^{+}\right)}^{2} d \tau \\
& \leq \frac{1}{2} \sup _{0 \leq \tau \leq t}\|w(\cdot, \tau)\|_{L^{2}\left(R^{+}\right)}^{2}+\alpha_{0}+\alpha_{3} \int_{0}^{t}\|w(\cdot, \tau)\|_{L^{2}\left(R^{+}\right)}^{2} d \tau
\end{aligned}
$$

for any $t \in(0, T]$, where $\alpha_{j}, j=0,1,2,3$, are constants depending only on $T$ and $\|(\phi, h)\|_{V_{T}^{1}}$. Consequently, it transpires that

$$
\sup _{0 \leq t \leq T}\|w(\cdot, t)\|_{L^{2}\left(R^{+}\right)}+\int_{0}^{t} w_{x}^{2}(0, t) d t \leq \alpha_{T}\left(\|(\phi, h)\|_{V_{T}^{1}}\right) .
$$

Next, multiply both sides of (5.4) by $-2 w_{x x}-w^{2}$, integrate over $R^{+}$and $(0, t)$ with respect to $x$ and $t$, respectively, and integrate by parts appropriately to reach the formula

$$
\begin{aligned}
& \int_{0}^{\infty} w_{x}^{2}(x, t) d x+\int_{0}^{t} w_{x x}^{2}(0, \tau) d \tau=\int_{0}^{\infty}\left|D_{x} \phi^{*}(x)\right|^{2} d x+\frac{1}{3} \int_{0}^{\infty} w^{3}(x, t) d x \\
& -5 \int_{0}^{t} \int_{0}^{\infty} v_{x}^{2}(x, \tau) w_{x}(x, \tau) d x d \tau-2 \int_{0}^{t} \int_{0}^{\infty} v_{x x}(x, \tau) w(x, \tau) w_{x}(x, \tau) d x d \tau \\
& -2 \int_{0}^{t} \int_{0}^{\infty} v_{x x}(x, \tau) v(x, \tau) w_{x}(x, \tau) d x d \tau+\frac{2}{3} \int_{0}^{t} \int_{0}^{\infty} v_{x}(x, \tau) w^{3}(x, \tau) d x d \tau \\
& +\int_{0}^{t} \int_{0}^{\infty} v_{x}(x, \tau) v(x, \tau) w^{2}(x, \tau) d x d \tau-\int_{0}^{t} v(0, \tau) w_{x}^{2}(0, \tau) d \tau \\
& -\int_{0}^{t} v(0, \tau) v_{x}(0, \tau) w_{x}(0, \tau) d \tau-2 \int_{0}^{t} Y(0, \tau) w_{x}(0, \tau) d \tau \\
& +2 \int_{0}^{t} \int_{0}^{\infty} Y_{x}(x, \tau) w_{x}(x, \tau) d x d \tau-\int_{0}^{t} \int_{0}^{\infty} Y(x, \tau) w^{2}(x, \tau) d x d \tau \\
& -2 \int_{0}^{t} \int_{0}^{\infty} g_{x x}(x, \tau) w(x, \tau) w_{x}(x, \tau) d x d \tau-3 \int_{0}^{t} \int_{0}^{\infty} g_{x}(x, \tau) w_{x}^{2}(x, \tau) d x d \tau \\
& -\int_{0}^{t} g(0, \tau) w_{x}^{2}(0, \tau) d \tau+\frac{2}{3} \int_{0}^{t} \int_{0}^{\infty} g_{x}(x, \tau) w^{3}(x, \tau) d x d \tau \\
& -2 \int_{0}^{t} \int_{0}^{\infty} g_{x x}(x, \tau) w_{x}(x, \tau) d x d \tau-4 \int_{0}^{t} \int_{0}^{\infty} g_{x}(x, \tau) v_{x}(x, \tau) w_{x}(x, \tau) d x d \tau \\
& -2 \int_{0}^{t} \int_{0}^{\infty} g(x, \tau) v_{x x}(x, \tau) w_{x}(x, \tau) d x d \tau \\
& -2 \int_{0}^{t}\left[g_{x}(0, \tau) v(0, \tau)+g(0, \tau) v_{x}(0, \tau)\right] w_{x}(0, \tau) d \tau
\end{aligned}
$$


For any $t \in(0, T]$, the terms on the right-hand side of equation (5.9) may be estimated as follows:

$$
\begin{aligned}
& \left|\int_{0}^{t} \int_{0}^{\infty} v_{x}(x, \tau) w^{3}(x, \tau) d x d \tau\right| \\
& \leq \int_{0}^{t}\|w(\cdot, \tau)\|_{H^{1}\left(R^{+}\right)}^{2}\left\|v_{x}(\cdot, \tau)\right\|_{L^{2}\left(R^{+}\right)}\|w(\cdot, \tau)\|_{L^{2}\left(R^{+}\right)} d \tau \\
& \leq\|v\|_{Z_{T}^{1}} \sup _{0 \leq \tau \leq t}\|w(\cdot, \tau)\|_{L^{2}\left(R^{+}\right)} \int_{0}^{t}\|w(\cdot, \tau)\|_{H^{1}\left(R^{+}\right)}^{2} d \tau ; \\
& \left|\int_{0}^{t} \int_{0}^{\infty} v(x, \tau) v_{x}(x, \tau) w^{2}(x, \tau) d x d \tau\right| \\
& \leq \int_{0}^{t}\|w(\cdot, \tau)\|_{H^{1}\left(R^{+}\right)}^{2}\|v(\cdot, \tau)\|_{L^{2}\left(R^{+}\right)}\left\|v_{x}(\cdot, \tau)\right\|_{L^{2}\left(R^{+}\right)} d \tau \\
& \leq\|v\|_{Z_{T}^{1}}^{2} \int_{0}^{t}\|w(\cdot, \tau)\|_{H^{1}\left(R^{+}\right)}^{2} d \tau ; \\
& \left|\int_{0}^{t} \int_{0}^{\infty} v_{x}^{2}(x, \tau) w_{x}(x, \tau) d x d \tau\right| \\
& \leq \int_{0}^{t}\left\|v_{x}(\cdot, \tau)\right\|_{L^{\infty}\left(R^{+}\right)}\left\|v_{x}(\cdot, \tau)\right\|_{L^{2}\left(R^{+}\right)}\left\|w_{x}(\cdot, \tau)\right\|_{L^{2}\left(R^{+}\right)} d \tau \\
& \leq \sup _{0 \leq \tau \leq t}\left\|v_{x}(\cdot, \tau)\right\|_{L^{2}\left(R^{+}\right)}\left(\int_{0}^{t}\left\|v_{x}(\cdot, \tau)\right\|_{L^{\infty}\left(R^{+}\right)}^{4} d \tau\right)^{1 / 4}\left(\int_{0}^{t}\left\|w_{x}(\cdot, \tau)\right\|_{L^{2}\left(R^{+}\right)}^{4 / 3} d \tau\right)^{3 / 4} \\
& \leq C_{T}\|v\|_{Z_{T}^{1}}^{2}\left(\int_{0}^{t}\|w(\cdot, \tau)\|_{H^{1}\left(R^{+}\right)}^{2} d \tau\right)^{1 / 2} \\
& \left|\int_{0}^{t} \int_{0}^{\infty} v_{x x}(x, \tau) w(x, \tau) w_{x}(x, \tau) d x d \tau\right| \\
& \leq \int_{0}^{t}\left\|v_{x x}(\cdot, \tau)\right\|_{L^{\infty}\left(R^{+}\right)}\|w(\cdot, \tau)\|_{H^{1}\left(R^{+}\right)}^{2} d \tau \\
& \leq\left(\int_{0}^{t}\left\|v_{x x}(\cdot, \tau)\right\|_{L^{\infty}\left(R^{+}\right)}^{4} d \tau\right)^{1 / 4}\left(\int_{0}^{t}\|w(\cdot, \tau)\|_{H^{1}\left(R^{+}\right)}^{8 / 3}\right)^{3 / 4} \\
& \leq C_{T}\|h\|_{H^{5 / 6}(0, T)} \sup _{0 \leq \tau \leq t}\|w(\cdot, \tau)\|_{H^{1}\left(R^{+}\right)}\left(\int_{0}^{t}\|w(\cdot, \tau)\|_{H^{1}\left(R^{+}\right)}^{4 / 3}\right)^{3 / 4} \\
& \leq C_{T}\|h\|_{H^{5 / 6}(0, T)} \sup _{0 \leq \tau \leq t}\|w(\cdot, \tau)\|_{H^{1}\left(R^{+}\right)}\left(\int_{0}^{t}\|w(\cdot, \tau)\|_{H^{1}\left(R^{+}\right)}^{2}\right)^{1 / 2} ;
\end{aligned}
$$




$$
\begin{aligned}
& \left|\int_{0}^{t} \int_{0}^{\infty} v(x, \tau) v_{x x}(x, \tau) w_{x}(x, \tau) d x d \tau\right| \\
& \quad \leq \int_{0}^{t}\left\|v(\cdot, \tau) v_{x x}(\cdot, \tau)\right\|_{L^{2}\left(R^{+}\right)}\left\|w_{x}(\cdot, \tau)\right\|_{L^{2}\left(R^{+}\right)} d \tau \\
& \quad \leq\left(\int_{0}^{t}\left\|v(\cdot, \tau) v_{x x}(\cdot, \tau)\right\|_{L^{2}\left(R^{+}\right)}^{2} d \tau\right)^{1 / 2}\left(\int_{0}^{t}\left\|w_{x}(\cdot, \tau)\right\|_{L^{2}\left(R^{+}\right)}^{2} d \tau\right)^{1 / 2} \\
& \quad \leq \int_{0}^{t}\left\|w_{x}(\cdot, \tau)\right\|_{L^{2}\left(R^{+}\right)}^{2} d \tau+\int_{0}^{\infty} \sup _{0 \leq \tau \leq t} v^{2}(x, \tau) \int_{0}^{t} v_{x x}^{2}(x, \tau) d \tau d x \\
& \quad \leq \int_{0}^{t}\left\|w_{x}(\cdot, \tau)\right\|_{L^{2}\left(R^{+}\right)}^{2} d \tau+\sup _{0<x<+\infty} \int_{0}^{t} v_{x x}^{2}(x, \tau) d \tau \int_{0}^{\infty} \sup _{0 \leq \tau \leq t} v^{2}(x, \tau) d x \\
& \quad \leq \int_{0}^{t}\left\|w_{x}(\cdot, \tau)\right\|_{L^{2}\left(R^{+}\right)}^{2} d \tau+\|v\|_{Z_{T}^{1}}^{4}
\end{aligned}
$$

and

$$
\begin{aligned}
\left|\int_{0}^{\infty} w^{3}(x, t) d x\right| & \leq\|w(\cdot, t)\|_{H^{1}\left(R^{+}\right)}\|w(\cdot, t)\|_{L^{2}}^{2} \\
& \leq \frac{1}{4}\|w(\cdot, t)\|_{H^{1}\left(R^{+}\right)}^{2}+\|w(\cdot, t)\|_{L^{2}\left(R^{+}\right)}^{4} .
\end{aligned}
$$

In a similar vein, one obtains the following inequalities:

$$
\begin{gathered}
\int_{0}^{t} \int_{0}^{\infty}|Y(x, \tau)| w^{2}(x, \tau) d x d \tau \leq C_{T}\|(\phi, h)\|_{V_{T}^{1}}^{2} \int_{0}^{t}\|w\|_{L^{2}\left(R^{+}\right)}^{2} d \tau \\
\int_{0}^{t} \int_{0}^{\infty}\left|Y_{x}(x, \tau) w_{x}(x, \tau)\right| d x d \tau \leq C_{T}\|(\phi, h)\|_{V_{T}^{1}}\left(\int_{0}^{t}\|w(\cdot, \tau)\|_{H^{1}\left(R^{+}\right)}^{2} d \tau\right)^{1 / 2} \\
\int_{0}^{t} \int_{0}^{\infty}\left|g_{x}(x, \tau) w^{3}(x, \tau)\right| d x d \tau \\
\leq|h(0)| \int_{0}^{t}\|w(\cdot, \tau)\|_{H^{1}\left(R^{+}\right)}\|w(\cdot, \tau)\|_{L^{2}\left(R^{+}\right)}^{2} d \tau \\
\leq\|h\|_{H^{2 / 3}(0, T)} \sup _{0 \leq \tau \leq t}\|w(\cdot, \tau)\|_{L^{2}\left(R^{+}\right)}^{2} \int_{0}^{t}\|w(\cdot, \tau)\|_{H^{1}\left(R^{+}\right)} d \tau \\
\int_{0}^{t} \int_{0}^{\infty}\left|g_{x x}(x, \tau) w(x, \tau) w_{x}(x, \tau)\right| d x d \tau \\
\leq C_{T}\|h\|_{H^{2 / 3}(0, T)} \sup _{0 \leq \tau \leq t}\|w(\cdot, \tau)\|_{L^{2}\left(R^{+}\right)} \int_{0}^{t}\left\|w_{x}(\cdot, \tau)\right\|_{L^{2}\left(R^{+}\right)} d \tau \\
\int_{0}^{t} \int_{0}^{\infty}\left|g_{x}(x, \tau) w_{x}^{2}(x, \tau)\right| d x \leq C_{T}\|h\|_{H^{2 / 3}(0, T)} \int_{0}^{t}\left\|w_{x}(\cdot, \tau)\right\|_{L^{2}\left(R^{+}\right)}^{2} d \tau
\end{gathered}
$$




$$
\begin{aligned}
& \int_{0}^{t} \int_{0}^{\infty}\left|(g(x, \tau) v(x, \tau))_{x} w^{2}(x, \tau)\right| d x d \tau \leq C_{t}\|v\|_{Z_{T}^{1}} \int_{0}^{t}\|w(\cdot, \tau)\|_{L^{2}\left(R^{+}\right)}^{2} d \tau \\
& \int_{0}^{t} \int_{0}^{\infty}\left|g(x, \tau) v_{x x}(x, \tau) w_{x}(x, \tau)\right| d x d \tau \\
& \quad \leq C_{T}|h(0)|\left(\int_{0}^{\infty} \sup _{0 \leq \tau \leq t}\left|v_{x x}(x, \tau)\right|^{2} d x\right)^{1 / 2}\left(\int_{0}^{t} \int_{0}^{\infty} w_{x}^{2}(x, \tau) d x d \tau\right)^{1 / 2}
\end{aligned}
$$

and

$$
\int^{t} \int_{0}^{\infty}\left|\left(g_{x} v\right)_{x} w_{x}\right| d x d \tau \leq C_{T}\|v\|_{Z_{T}^{1}} \int_{0}^{t}\left\|w_{x}\right\|_{L^{2}\left(R^{+}\right)} d \tau .
$$

In addition, the integrals corresponding to boundary traces need to be bounded:

$$
\begin{gathered}
\left|\int_{0}^{t} v(0, \tau) w_{x}^{2}(0, \tau) d \tau\right| \leq \sup _{0 \leq \tau \leq t}|v(0, \tau)| \int_{0}^{t} w_{x}^{2}(0, \tau) d \tau \\
\leq\|v\|_{Z_{T}^{1}} \int_{0}^{t} w_{x}^{2}(0, \tau) d \tau ; \\
\left|\int_{0}^{t} v(0, \tau) v_{x}(0, \tau) w_{x}(0, \tau) d \tau\right| \\
\leq\|v\|_{Z_{T}^{1}}\left(\int_{0}^{t} v_{x}^{2}(0, \tau) d \tau\right)^{1 / 2}\left(\int_{0}^{t} w_{x}^{2}(0, \tau) d \tau\right)^{1 / 2} \\
\leq\|v\|_{Z_{T}^{1}}^{2}\left(\int_{0}^{t} w_{x}^{2}(0, \tau) d \tau\right)^{1 / 2} ; \\
\int_{0}^{t}\left|Y(0, \tau) w_{x}(0, \tau)\right| d \tau \leq 3\left(\|h\|_{H^{2 / 3}(0, T)}+\|h\|_{H^{2 / 3}(0, T)}^{2}\right) \\
\times\left(\int_{0}^{t}\left|w_{x}(0, \tau)\right|^{2} d \tau\right)^{1 / 2} ; \\
\left.\int_{0}^{t}|g(0, \tau)| w_{x}^{2}(0, \tau)|d \tau \leq| h(0)\left|\int_{0}^{t}\right| w_{x}(0, \tau)\right|^{2} d \tau
\end{gathered}
$$

and

$$
\begin{gathered}
\int_{0}^{t}\left|g_{x}(0, \tau)\left[v(0, \tau)+v_{x}(0, \tau)\right] w_{x}(0, \tau)\right| d \tau \leq C_{T}|h(0)|\|v\|_{Z_{T}^{1}} \\
\times\left(\int_{0}^{t}\left|w_{x}(0, \tau)\right|^{2} d \tau\right)^{1 / 2} .
\end{gathered}
$$


Combining the above estimates yields the inequality

$$
\begin{gathered}
\|w(\cdot, t)\|_{H^{1}\left(R^{+}\right)}^{2}+\int_{0}^{t} w_{x x}^{2}(0, \tau) d \tau \\
\leq \frac{1}{2} \sup _{0 \leq \tau \leq t}\|w(\cdot, \tau)\|_{H^{1}\left(R^{+}\right)}^{2}+\alpha_{1}+\alpha_{2} \int_{0}^{t}\|w\|_{H^{1}\left(R^{+}\right)}^{2} d \tau,
\end{gathered}
$$

valid for any $t \in[0, T]$, where the $\alpha_{j}, j=1,2$, depend only on $T,\|\phi\|_{H^{1}\left(R^{+}\right)}$and $\|h\|_{H^{5 / 6}\left(R^{+}\right)}$. As before, there obtains from this integral inequality the bound

$$
\sup _{0 \leq t \leq T}\|w(\cdot, t)\|_{H^{1}\left(R^{+}\right)}+\left(\int_{0}^{T} w_{x x}^{2}(0, t) d t\right)^{1 / 2} \leq \alpha_{T}\left(\|(\phi, h)\|_{V_{T}^{1}}\right) .
$$

The proof of Proposition 5.3 is complete.

Proof of Proposition 5.4. Observing that

$$
u_{x x x}=-u_{t}-u_{x}-u u_{x}
$$

we are naturally led to search for a global $L^{2}$-estimate on $u_{t}$ instead of attempting to derive a global $L^{2}$-estimate for $u_{x x x}$ directly. As observed already in [5, 6], there is a crucial advantage to this approach in terms of the boundary traces that arise in the analysis.

If $v=u_{t}$, then $v$ solves

$$
\left\{\begin{array}{l}
\partial_{t} v+\partial_{x} v+\partial_{x}(u v)+\partial_{x}^{3} v=0, \quad \text { for } x, t \geq 0 \\
v(x, 0)=\phi_{1}(x), \quad v(0, t)=h_{1}(t)
\end{array}\right.
$$

with $\phi_{1}(x)=-\phi^{\prime}(x)-\phi(x) \phi^{\prime}(x)-\phi^{\prime \prime \prime}(x)$ and $h_{1}(t)=h^{\prime}(t)$. We show that

$$
\sup _{t \in[0, T]}\|v(\cdot, t)\|_{L^{2}\left(R^{+}\right)} \leq \alpha_{T}\left(\|u\|_{Z_{T}^{1}}\right)\left(\left\|h_{1}\right\|_{H^{1 / 3}\left(R^{+}\right)}+\left\|\phi_{1}\right\|_{L^{2} R^{+}}\right),
$$

which, together with (5.2) is equivalent to the desired estimate (5.3).

Rewrite $v$ as $v=w+z$ with $w$ solving

$$
\begin{cases}w_{t}+w_{x}+w_{x x x}=0, & \text { for } x, t \geq 0, \\ w(x, 0)=\phi_{1}(x), & w(0, t)=h_{1}(t) .\end{cases}
$$

It follows that $z$ is a solution to the initial-boundary-value problem

$$
\left\{\begin{array}{l}
\partial_{t} z+\partial_{x} z+\partial_{x}(u z)+\partial_{x}^{3} z=-(u w)_{x}, \quad \text { for } x, t \geq 0, \\
z(x, 0)=0, \quad z(0, t)=0 .
\end{array} .\right.
$$

By Proposition 4.5, there is a $T$-dependent constant $C_{T}$ such that

$$
\begin{aligned}
& \sup _{0 \leq t \leq T}\|w(\cdot, t)\|_{L^{2}\left(R^{+}\right)}+\sup _{x \geq 0}\left(\int_{0}^{T}\left|w_{x}(x, t)\right|^{2} d t\right)^{1 / 2} \\
& \leq C_{T}\left(\left\|\phi_{1}\right\|_{L^{2}\left(R^{+}\right)}+\left\|h_{1}\right\|_{H^{1 / 3}(0, T)}\right) .
\end{aligned}
$$


Since

$$
\begin{aligned}
& \int_{0}^{T} \int_{0}^{\infty}\left|w_{x}(x, t) u(x, t)\right|^{2} d x d t=\int_{0}^{\infty} \int_{0}^{T}\left|w_{x}(x, t) u(x, t)\right|^{2} d t d x \\
& \quad \leq \int_{0}^{\infty} \sup _{0 \leq t \leq T}|u(x, t)|^{2} \int_{0}^{T}\left|w_{x}(x, t)\right|^{2} d t d x \\
& \quad \leq \sup _{x \geq 0} \int_{0}^{T}\left|w_{x}(x, t)\right|^{2} d t \int_{0}^{\infty} \sup _{0 \leq t \leq T}|u(x, t)|^{2} d x \\
& \quad \leq C_{T}\|u\|_{Z_{T}^{1}}^{2}\left(\left\|\phi_{1}\right\|_{L^{2}\left(R^{+}\right)}+\left\|h_{1}\right\|_{H^{1 / 3}(0, T)}\right)^{2}
\end{aligned}
$$

and

$$
\begin{aligned}
\int_{0}^{T} & \int_{0}^{\infty}\left|w(x, t) u_{x}(x, t)\right|^{2} d x d t \leq \int_{0}^{T} \sup _{x \in R^{+}}\left|u_{x}(x, t)\right|^{2} \int_{0}^{\infty}|w(x, t)|^{2} d x d t \\
& \leq \sup _{0 \leq t \leq T} \int_{0}^{\infty}|w(x, t)|^{2} d x \int_{0}^{T} \sup _{x \in R^{+}}\left|u_{x}(x, t)\right|^{2} d t \\
& \leq C_{T}\|u\|_{Z_{T}^{1}}^{2}\left(\left\|\phi_{1}\right\|_{L^{2}\left(R^{+}\right)}+\left\|h_{1}\right\|_{H^{1 / 3}(0, T)}\right)^{2},
\end{aligned}
$$

it is seen that

$$
\left(\int_{0}^{T}\left\|(u w)_{x}\right\|_{L^{2}\left(R^{+}\right)}^{2} d t\right)^{1 / 2} \leq C_{T}\|u\|_{Z_{T}^{1}}\left(\left\|\phi_{1}\right\|_{L^{2}\left(R^{+}\right)}+\left\|h_{1}\right\|_{H^{1 / 3}(0, T)}\right)
$$

for any $T>0$.

Next multiply both sides of the evolution equation in (5.12) by $2 z$ and integrate with respect to $x$ over $R^{+}$. After integration by parts, there appears

$$
\frac{d}{d t} \int_{0}^{\infty} z^{2}(x, t) d x+z_{x}^{2}(0, t)+\int_{0}^{\infty} u_{x}(x, t) z^{2}(x, t) d x=2 \int_{0}^{\infty} g(x, t) z(x, t) d x
$$

which holds for any $t \in[0, T]$, where $g=-(u w)_{x}$. As a result, it follows that

$$
\frac{d}{d t} \int_{0}^{\infty} z^{2}(x, t) d x \leq \int_{0}^{\infty} g^{2}(x, t) d x+\left(1+\sup _{x \in R^{+}}\left|u_{x}(x, t)\right|\right) \int_{0}^{\infty} z^{2}(x, t) d x .
$$

Using Gronwall's lemma, it is adduced that

$$
\begin{aligned}
\sup _{0 \leq t \leq T} \int_{0}^{\infty} z^{2}(x, t) d x & \leq \int_{0}^{t} e^{\int_{s}^{t}\left(1+\sup _{y \geq 0}\left|u_{x}(y, \tau)\right|\right) d \tau} \int_{0}^{\infty} g^{2}(x, s) d x d s \\
& \leq \alpha_{T}\left(\|u\|_{Z_{T}^{1}}\right) \int_{0}^{T} \int_{0}^{\infty} g^{2}(x, s) d x d s,
\end{aligned}
$$

which, together with (5.13) and (5.14), yields (5.11). The proof is complete.

Next, a global a priori bound in $H^{s}\left(R^{+}\right)$is obtained for solutions of 4.1) when $s>3$.

Theorem 5.5. For given $T>0$ and $s=3 m+s^{\prime}$ with $0<s^{\prime} \leq 3$ and $m \geq 1$, there exists a T-dependent and continuous non-decreasing function $\alpha_{T}: R^{+} \rightarrow R^{+}$such 
that for any smooth solution $u$ of (4.1),

$$
\begin{gathered}
\sup _{0 \leq t \leq T}\left\|\partial_{t}^{m} u(\cdot, t)\right\|_{H^{s^{\prime}}\left(R^{+}\right)}+\sum_{k=0}^{m-1} \sup _{0 \leq t \leq T}\left\|\partial_{t}^{k} u(\cdot, t)\right\|_{H^{3}\left(R^{+}\right)} \\
\leq \alpha_{T}\left(\|(\phi, h)\|_{H^{s}\left(R^{+}\right) \times H^{(s+1) / 3}(0, T)}\right) .
\end{gathered}
$$

Proof. We only prove Theorem 5.5 for $m=1$. The general case follows by induction. Let $v=u_{t}$. Then $v$ is a solution of

$$
\left\{\begin{array}{l}
\partial_{t} v+\partial_{x} v+\partial_{x}(u v)+\partial_{x}^{3} v=0, \quad \text { for } x, t \geq 0 \\
v(x, 0)=\phi_{1}(x), \quad v(0, t)=h_{1}(t) .
\end{array}\right.
$$

The problem (5.15) is linear, but with $u \in Z_{T}^{3}$ as a variable coefficient. Applying the proof of Proposition 5.3, the following estimate emerges for sufficiently regular solutions of [5.15):

$$
\sup _{0 \leq t \leq T}\|v(\cdot, t)\|_{L^{2}\left(R^{+}\right)} \leq C_{T}\|u\|_{Z_{T}^{1}}\left(\left\|\phi_{1}\right\|_{L^{2}\left(R^{+}\right)}+\left\|h_{1}\right\|_{H^{1 / 3}(0, T)}\right) .
$$

Secondly, by using Proposition 6.1 in the next section, which, as mentioned before, is proved independently of the considerations in this section, we also have

$$
\sup _{t \in[0, T]}\|v(\cdot, t)\|_{H^{1}\left(R^{+}\right)} \leq \alpha_{T}\left(\|u\|_{Z_{T}^{1}}\right)\left(\left\|\phi_{1}\right\|_{H^{1}\left(R^{+}\right)}+\left\|h_{1}\right\|_{H^{2 / 3}(0, T)}\right) .
$$

The following estimates hold by interpolation:

$$
\begin{aligned}
\sup _{t \in[0, T]}\|v(\cdot, t)\|_{H^{s^{\prime}}\left(R^{+}\right)} & \leq \alpha_{T}\left(\|u\|_{Z_{T}^{1}}\right)\|(\phi, h)\|_{H^{s}\left(R^{+}\right) \times H^{(s+1) / 3}(0, T)} \\
& \leq \alpha_{T}\left(\|(\phi, h)\|_{H^{s}\left(R^{+}\right) \times H^{(s+1) / 3}(0, T)}\right)
\end{aligned}
$$

for any $s^{\prime}$ with $0 \leq s^{\prime} \leq 1$. When $s^{\prime}>1$, using Proposition 6.1 directly gives

$$
\begin{aligned}
\sup _{t \in[0, T]}\|v(\cdot, t)\|_{H^{s^{\prime}}\left(R^{+}\right)} & \leq \alpha_{T}\left(\|u\|_{Z_{T}^{3}}\right)\|(\phi, h)\|_{H^{s}\left(R^{+}\right) \times H^{(s+1) / 3}(0, T)} \\
& \leq \alpha_{T}\left(\|(\phi, h)\|_{H^{s}\left(R^{+}\right) \times H^{(s+1) / 3}(0, T)}\right) .
\end{aligned}
$$

Thus (5.15) holds for $m=1$. As already indicated, the remainder of the proof follows by an induction that is analogous to the argument just presented for the case $m=1$.

As an immediate consequence of Theorem 5.1, Theorem 5.5 and the local wellposedness results established in Section 4, the following global well-posedness result for the initial-boundary-value problem (4.1) is obtained.

Theorem 5.6. Let $T>0$ and $s \geq 1$ be given. Then for any $s$-compatible $(\phi, h) \in$ $H^{s}\left(R^{+}\right) \times H^{(7+3 s) / 12}(0, T)$ when $1 \leq s \leq 3$ and for any $s$-compatible $(\phi, h) \in$ $H^{s}\left(R^{+}\right) \times H^{(s+1) / 3}(0, T)$ when $s>3$, the problem $\sqrt{4.1)}$ admits a unique solution $u \in Z_{T}^{s} \cap C\left([0, T] ; H^{s}\left(R^{+}\right)\right)$with $\partial_{t}^{k} u \in C\left([0, T] ; H^{s-3 k}\left(R^{+}\right)\right)$for $k=0,1, \cdots,[s / 3]$. 


\section{TAYLOR SERIES EXPANSION}

Having established local and global well-posedness results, interest naturally turns to related issues. Here we focus on the mapping that takes compatible pairs of initial- and boundary-data into associated solutions and inquire about the regularity of this correspondence. For given $T>0$ and $s \geq 0$, let $X_{T}^{s}$ be the collection of all $s$-compatible functions $(\phi, h) \in H^{s}\left(R^{+}\right) \times H^{(s+1) / 3}(0, T)$. By its definition, $X_{T}^{s}$ is a linear vector subspace of $H^{s}\left(R^{+}\right) \times H^{(s+1) / 3}(0, T)$ only when $0 \leq s \leq 7 / 2$. When $s$ is in this range, we consider $X_{T}^{s}$ as a Banach space with norm induced by that of $H^{s}\left(R^{+}\right) \times H^{(s+1) / 3}(0, T)$. For any $s>3 / 4$, the results established in Sections 4 and 5 show that the initial-boundary-value problem (4.1) defines a nonlinear map $K_{I}$ from the space $X_{T}^{s}$ to the space $Z_{T}^{s}$. For $T>0$, let $\mathcal{D}_{s}^{T}=\mathcal{D}_{s}^{T}\left(K_{I}\right)$ denote the domain of the map $K_{I}$ in the space $X_{T}^{s}$. An element $g=(\phi, h)$ belongs to $\mathcal{D}_{s}^{T}$ if $(\phi, h) \in X_{T}^{s}$ and the associated solution $u$ of (4.1) with auxiliary data $(\phi, h)$ exists at least on the time interval $[0, T]$. Obviously, $\mathcal{D}_{s}^{T}$ is not empty since $0 \in \mathcal{D}_{s}^{T}$. Because of the global well-posedness of (4.1) for $s \geq 3$, it is clear that $\mathcal{D}_{s}^{T}=X_{T}^{s}$ in this case. From the proofs of the results presented in Section 4, the map $K_{I}$ is known to be Lipschitz continuous from $\mathcal{D}_{s}^{T}$ to $Z_{T}^{s}$. In this section it is shown that $K_{I}$ has far stronger regularity. More precisely, when $3 / 4<s \leq 7 / 2$, for any $g \in \mathcal{D}_{s}^{T}$, there exists an $\eta>0$ such that for any $w \in X_{T}^{s}$ with $\|w\|_{X_{T}^{s}} \leq \eta$, we have $g+w \in \mathcal{D}_{s}^{T}$ and $K_{I}(g+w)$ has the following Taylor series expansion:

$$
K_{I}(g+w)=K_{I}(g)+\sum_{n=1}^{\infty} \frac{K_{I}^{(n)}(g)\left[w^{n}\right]}{n !}
$$

where $K_{I}^{(n)}(g)$ is the $n$-th order Fréchet derivative of $K_{I}$ evaluated at $g$ and the series converges strongly in the space $Z_{T}^{s}$. In other words, the map $K_{I}$ is analytic. In case $s>7 / 2$, the Taylor series expansion does not hold in the form just presented since the space $X_{T}^{s}$ is no longer a vector space. In this situation, consideration is given to an initial-boundary-value problem for a general $m$-nonlinear system which includes (4.1) as a special case. It will be shown that the corresponding nonlinear map $\mathcal{K}_{I}$ is analytic.

To begin, we present a well-posedness result for the linear, variable-coefficient, initial-boundary-value problem

$$
\left\{\begin{array}{l}
\partial_{t} u+\partial_{x} u+\partial_{x}(a u)+\partial_{x}^{3} u=\partial_{x}(f g), \quad \text { for } x \geq 0,0 \leq t \leq T, \\
u(x, 0)=\phi(x), \quad u(0, t)=h(t),
\end{array}\right.
$$

for the linearized KdV equation. This result was already used in Section 5 and will also play an important role in establishing analyticity of the map $K_{I}$.

Proposition 6.1. Let $T>0$ and $s \in(3 / 4,3]$ be given. Suppose that $a, f, g \in Z_{T}^{s}$. Then, for any $(\phi, h) \in X_{T}^{s}$, (6.1) admits a unique solution $u \in Z_{T}^{s}$ satisfying

$$
\|u\|_{Z_{T}^{s}} \leq \alpha_{T}\left(\|a\|_{Z_{T}^{s}}\right)\left(\|f\|_{Z_{T}^{1}}\|g\|_{Z_{T}^{s}}+\|(\phi, h)\|_{X_{T}^{s}}\right)
$$

where $\alpha_{T}: R^{+} \rightarrow R^{+}$is a $T$-dependent and continuous non-decreasing function which is independent of $f, g$ and $(\phi, h)$. 
Proof. The proof is similar to that of Theorem 4.8 and consequently we content ourselves with a sketch. For given $(\phi, h) \in X_{T}^{s}, \beta \in(0, T]$ and $r>0$, let

$$
S_{\beta, r}=\left\{w \in Z_{\beta}^{s}: \quad w(x, 0)=\phi(x), w(0, t)=h(t),\|w\|_{Z_{\beta}^{s}} \leq r\right\} .
$$

For given $a, f, g \in Z_{T}^{s}$, consider the map $\Gamma: S_{\beta, r} \rightarrow Z_{T}^{s}$ defined for $v \in S_{\beta, r}$ by

$$
u=\Gamma(v),
$$

where $u$ is the unique solution of

$$
\left\{\begin{array}{l}
u_{t}+u_{x}+u_{x x x}=-(a v)_{x}+(f g)_{x}, \quad \text { for } x \geq 0,0 \leq t \leq T, \\
u(x, 0)=\phi(x), \quad u(0, t)=h(t) .
\end{array}\right.
$$

Applying Lemmas $4.1-4.6$, one can show that there is a constant $C$ depending on $r$ and $T$, but independent of $v$, such that

$$
\lambda_{\beta, s}(\Gamma(v)) \leq C\left(\|g\|_{Z_{T}^{s}}\|f\|_{Z_{T}^{s}}+\|(\phi, h)\|_{X_{T}^{s}}\right)+C\left(\beta^{1 / 4}+\beta^{1 / 2}\right) \lambda_{T, s}(a) \lambda_{\beta, s}(v) .
$$

Hence if we choose $r$ so that

$$
r=2 C\left(\|g\|_{Z_{T}^{s}}\|f\|_{Z_{T}^{s}}+\|(\phi, h)\|_{X_{T}^{s}}\right),
$$

then there is a unique choice $\tilde{\beta}$ for which

$$
r C\left(\tilde{\beta}^{1 / 4}+\tilde{\beta}^{1 / 2}\right) \lambda_{T, s}(a)=1 / 2 .
$$

If we define $\beta=\min \{T, \tilde{\beta}\}$, then

$$
\lambda_{\beta, s}(\Gamma(v)) \leq r
$$

for any $v \in S_{\beta, r}$. Moreover, for any $v_{1}, v_{2} \in S_{\beta, r}$,

$$
\lambda_{\beta, r}\left(\Gamma\left(v_{1}\right)-\Gamma\left(v_{2}\right)\right) \leq \frac{1}{2} \lambda_{\beta, r}\left(v_{1}-v_{2}\right) .
$$

With such a choice of $r$ and $\beta, \Gamma$ is a contraction map from $S_{\beta, r}$ to $S_{\beta, r}$. Its unique fixed point is the desired solution of (6.1) on the temporal interval $0 \leq t \leq \beta$. However, since the value of $\beta$ is chosen according to (6.3) and (6.4) which only depends on $\|a\|_{Z_{T}^{s}},\|g\|_{Z_{T}^{s}}$ and $\|f\|_{Z_{T}^{s}}$, a standard iteration extends the solution to the entire interval $0 \leq t \leq T$. The proof is complete.

The major step in the present development is to show that for $T>0$ and $s \in(3 / 4,3], \mathcal{D}_{s}^{T}$ is an open set in the space $X_{T}^{s}$ and that the nonlinear map $K_{I}$ is analytic from $\mathcal{D}_{s}^{T}$ to $Z_{T}^{s}$.

The following formal calculation is instructive. If $K_{I}$ is an analytic mapping from $\mathcal{D}_{s}^{T}$ to $Z_{T}^{s}$, then, for $n=0,1,2, \cdots$, its $n$-th order Fréchet derivative $K_{I}^{(n)}(g)$ at $g \in \mathcal{D}_{s}^{T}$ exists and is the symmetric, $n$-linear map from the $n$-fold product $X_{T}^{s} \times \cdots \times X_{T}^{s}$ to $Z_{T}^{s}$ given as

$$
K_{I}^{(n)}(g)\left[w_{1}, \cdots, w_{n}\right]=\left\{\frac{\partial^{n}}{\partial \xi_{1} \cdots \partial \xi_{n}} K_{I}\left(g+\sum_{k=1}^{n} \xi_{k} w_{k}\right)\right\}_{0, \cdots, 0}
$$

for any $w_{1}, w_{2}, \cdots, w_{n} \in X_{T}^{s}$. The homogeneous polynomial $K_{I}^{(n)}(g)\left[w^{n}\right]$ of degree $n$ induced by $K_{I}^{(n)}(g)$ evaluated at $w^{n}=(w, w, \cdots, w)$ (n-components) is

$$
K_{I}^{(n)}(g)\left[w^{n}\right]=\left\{\frac{d^{n}}{d \xi^{n}} K_{I}(g+\xi w)\right\}_{\xi=0}
$$


where $w=\left(w_{\phi}, w_{h}\right) \in X_{T}^{s}$. If we define $y_{n}$ by

$$
y_{n}=K_{I}^{(n)}(g)\left[w^{n}\right],
$$

then it is formally ascertained that $\left(y_{1}, y_{2}, \cdots, y_{n}\right)$ solves the system

$$
\left\{\begin{array}{l}
\partial_{t} y_{1}+\partial_{x} y_{1}+\partial_{x}\left(u y_{1}\right)+\partial_{x}^{3} y_{1}=0, \quad \text { for } x \geq 0,0 \leq t \leq T, \\
y_{1}(x, 0)=w_{\phi}(x), \quad y_{1}(0, t)=w_{h}(t)
\end{array}\right.
$$

and

$$
\left\{\begin{array}{l}
\partial_{t} y_{k}+\partial_{x} y_{k}+\partial_{x}\left(u y_{k}\right)+\partial_{x}^{3} y_{k}=-\frac{1}{2} \sum_{j=1}^{k-1}\left(\begin{array}{c}
k \\
j
\end{array}\right) \partial_{x}\left(y_{j} y_{k-j}\right), \\
y_{k}(x, 0)=0, \quad y_{k}(0, t)=0,
\end{array}\right.
$$

for $x \geq 0,0 \leq t \leq T$ and $2 \leq k \leq n$, where $u=K_{I}(g) \equiv y_{0}$ and $w=\left(w_{\phi}, w_{h}\right) \in X_{T}^{s}$.

On the other hand, for any $g=(\phi, h) \in \mathcal{D}_{s}^{T}$, let $u=K_{I}(g)$ and consider solving the linear system (6.5)- (6.6). It follows from Proposition 6.1 that the system (6.5)(6.6) may be used to define a homogeneous polynomial of degree $n$ which maps $X_{T}^{s}$ to $Z_{T}^{s}$ as described in the following proposition.

Proposition 6.2. Let $T>0,3 / 4<s \leq 3$ and $g \in \mathcal{D}_{s}^{T}=\mathcal{D}_{s}^{T}\left(K_{I}\right)$ be given and let $u=K_{I}(g)$. Then the system (6.5)-(6.6) defines a homogeneous polynomial $K_{I}^{(n)}(g)\left[w^{n}\right]$ of degree $n$ from $X_{T}^{s}$ to $Z_{T}^{s}$. Moreover, there exists a constant $c_{3}$ such that

$$
\left\|y_{n}\right\|_{Z_{T}^{s}} \leq c_{3}^{n} n !\|w\|_{X_{T}^{s}}^{n}
$$

for any $n \geq 2$, where $c_{3}=c_{3}\left(T,\|u\|_{Z_{T}^{s}}\right)$, and it may be that $c_{3} \rightarrow+\infty$ as $T \rightarrow+\infty$ or as $\|u\|_{Z_{T}^{s}} \rightarrow+\infty$, but in any case $c_{3} \rightarrow 0$ if $T \rightarrow 0$, or if $\|u\|_{Z_{T}^{s}} \rightarrow 0$.

Proof. The proof is a straightforward consequence of Lemmas $4.1-4.6$ and Proposition 6.1 (see [64], Prop. 3.3 for details).

For $w \in X_{T}^{s}$, define a Taylor polynomial $P_{n}(w)$ of degree $n$ by

$$
P_{n}(w)=\sum_{k=0}^{n} \frac{K_{I}^{(k)}(g)\left[w^{k}\right]}{k !}=K_{I}(g)+\sum_{k=1}^{n} \frac{y_{k}}{k !},
$$

and a Taylor series by

$$
P(w)=\sum_{k=0}^{\infty} \frac{K_{I}^{(k)}(g)\left[w^{k}\right]}{k !} .
$$

Proposition 6.3. Let $T>0$ and $3 / 4<s \leq 3$ be given. For any $g=(\phi, h) \in \mathcal{D}_{s}^{T}$, there exists an $\eta>0$ depending only on $\left\|K_{I}(g)\right\|_{Z_{T}^{s}}$ such that the formal Taylor series (6.9) is uniformly convergent in the space $Z_{T}^{s}$ with respect to $w \in X_{T}^{s}$ with $\|w\|_{X_{T}^{s}} \leq \eta$. Moreover, if $v=P(w)$, then $v \in Z_{T}^{s}$ solves the problem

$$
\begin{cases}v_{t}+v_{x}+v v_{x}+v_{x x x}=0, & \text { for } x \geq 0, t \in(0, T], \\ v(x, 0)=\phi(x)+w_{\phi}(x), & v(0, t)=h(t)+w_{h}(t) .\end{cases}
$$


Proof. It is readily seen that the sequence $\left\{P_{n}(w)\right\}_{n=0}^{\infty}$ of Taylor polynomials is Cauchy in $Z_{T}^{s}$ uniformly for $w$ in the ball of radius $\eta$ in $X_{T}^{s}$ for suitable $\eta$. Indeed, because of Proposition 6.2, it transpires that for $m \geq n \geq 0$,

$$
\left\|P_{n}(w)-P_{m}(w)\right\|_{Z_{T}^{s}}=\left\|\sum_{k=n}^{m} \frac{y_{k}}{k !}\right\|_{Z_{T}^{s}} \leq \sum_{k=n}^{m} \frac{\left\|y_{k}\right\|_{Z_{T}^{s}}}{k !} \leq \sum_{k=n}^{m} c_{3}^{k}\|w\|_{X_{T}^{s}}^{k} .
$$

If $\eta$ is chosen so that

$$
\eta \leq 1 /\left(2 c_{3}\right)
$$

then for $w \in X_{T}^{s}$ with $\|w\|_{X_{T}^{s}} \leq \eta$, one has

$$
\left\|P_{n}(w)-P_{m}(w)\right\|_{Z_{T}^{s}} \leq \sum_{k=n}^{m} \frac{1}{2^{k}}
$$

which goes to zero uniformly as $n, m \rightarrow \infty$.

Since $\left\{P_{n}(w)\right\}_{n=0}^{\infty}$ is a Cauchy sequence in the space $Z_{T}^{s}$, it makes sense to define $v=P(w)$ as its limit as $n \rightarrow \infty$. Then $v \in Z_{T}^{s}$ and $v$ solves the initial-boundaryvalue problem (6.10). To see this, note first that

$$
\begin{array}{r}
v(x, 0)=\sum_{k=0}^{\infty} \frac{y_{k}(x, 0)}{k !}=u(x, 0)+y_{1}(x, 0)=\phi(x)+w_{\phi}(x), \\
v(0, t)=\sum_{k=0}^{\infty} \frac{y_{k}(0, t)}{k !}=u(0, t)+y_{1}(0, t)=h(t)+w_{h}(t) .
\end{array}
$$

Moreover, since the series $P(w)$ is absolutely convergent in the Banach algebra $Z_{T}^{s}$, it follows that

$$
\begin{aligned}
v^{2} & =\left(u+\sum_{k=1}^{\infty} \frac{y_{k}}{k !}\right)^{2}=u^{2}+2 \sum_{k=1}^{\infty} \frac{u y_{k}}{k !}+\left(\sum_{k=1}^{\infty} \frac{y_{k}}{k !}\right)^{2} \\
& =2\left(\frac{1}{2} u^{2}+\sum_{k=1}^{\infty} \frac{u y_{k}}{k !}+\frac{1}{2} \sum_{k=1}^{\infty} \frac{1}{k !} \sum_{n=0}^{k-1}\left(\begin{array}{c}
k \\
n
\end{array}\right) y_{n} y_{n-k}\right) .
\end{aligned}
$$

In consequence, we have

$$
\begin{aligned}
\partial_{t} v & +\frac{1}{2} \partial_{x}\left(v^{2}\right)+\partial_{x}^{3} v=\partial_{t} u+\sum_{k=1}^{\infty} \frac{\partial_{t} y_{k}}{k !}+\partial_{x}^{3} u \\
& +\sum_{k=1}^{\infty} \frac{\partial_{x}^{3} y_{k}}{k !}+\frac{1}{2} \partial_{x}\left(u^{2}\right)+\sum_{k=1}^{\infty}\left\{\frac{\partial_{x}\left(u y_{k}\right)}{k !}+\frac{1}{2 k !} \sum_{n=0}^{k-1}\left(\begin{array}{c}
k \\
n
\end{array}\right) \partial_{x}\left(y_{n} y_{n-k}\right)\right\} \\
= & \left(\partial_{t} u+\frac{1}{2} \partial_{x}\left(u^{2}\right)+\partial_{x}^{3} u\right)+\left(\partial_{t} y_{1}+\partial_{x}\left(u y_{1}\right)+\partial_{x}^{3} y_{1}\right)+ \\
+ & \sum_{k=2}^{\infty} \frac{1}{k !}\left\{\partial_{t} y_{k}+\partial_{x}\left(u y_{k}\right)+\frac{1}{2} \sum_{n=0}^{k-1}\left(\begin{array}{c}
k \\
n
\end{array}\right) \partial_{x}\left(y_{n} y_{n-k}\right)+\partial_{x}^{3} y_{k}\right\} \\
& =0 .
\end{aligned}
$$

The proof is complete. 
The following theorem is now readily adduced.

Theorem 6.4 (Analyticity). For any $T>0$ and $3 / 4<s \leq 3$ the nonlinear problem (4.1) establishes a map $K_{I}$ from the space $X_{T}^{s}$ to the space $Z_{T}^{s}$ having as its domain $\mathcal{D}_{s}^{T}$ a non-empty open subset of $X_{T}^{s}$. The map $K_{I}$ is analytic from $\mathcal{D}_{s}^{T}$ to $Z_{T}^{s}$ in the sense that for any $\phi \in \mathcal{D}_{s}^{T}$, there exists an $\eta>0$ such that for any $w \in X_{T}^{s}$ with $\|w\|_{X_{T}^{s}} \leq \eta$, the Taylor series expansion

$$
K_{I}(\phi+w)=\sum_{n=0}^{\infty} \frac{K_{I}^{(n)}(\phi)\left[w^{n}\right]}{n !}
$$

converges in the space $Z_{T}^{s}$. Moreover, the convergence is uniform with regard to $w$ in the aforementioned ball in $X_{T}^{s}$.

Remarks. 1. The above theorem holds also for $3<s<7 / 2$. Since its proof is similar to that for the system discussed below, a separate discussion is not included.

2. Since $0 \in \mathcal{D}_{s}^{T}$, there exists an $\eta>0$ depending on $T$ such that for any $g=(\phi, h) \in X_{T}^{s}$ with $\|g\|_{X_{T}^{s}} \leq \eta$, the problem (4.1) has a unique solution $u \in Z_{T}^{s}$ defined at least on the time interval $(0, T)$. Moreover, according to (6.8) and Proposition 6.2, $\eta \rightarrow \infty$ as $T \rightarrow 0$. The local well-posedness of the problem (4.1) thus follows as a corollary to Theorem 5.1. This provides an alternative approach to the well-posedness of (4.1): show first the analyticity of the map $K_{I}$ by establishing the solvability of the $n$-linear system (6.5)(6.6). One advantage of this approach is that it clearly shows the solution of the nonlinear problem (4.1) can be obtained by solving a sequence of linear problems.

3. We know already that (4.1) is globally well-posed in the space $X_{T}^{s}$ when $s \geq 3$. In case $3 / 4<s<3$, only local well-posedness has been proved in $X_{T}^{s}$ and the needed a priori estimates are not available. Of course, global well-posedness is valid for $1 \leq s<3$, but slightly stronger conditions on the boundary data are needed than is implied by membership in $X_{T}^{s}$. This raises the question of whether the corresponding solutions blow up in finite time or exist globally in the space $H^{s}\left(R^{+}\right)$. As an application of the analyticity of the map $K_{I}$, a partial answer to this question is forthcoming. For $(\phi, h) \in X_{\infty}^{s}$, the corresponding solution $u$ of (4.1) exists globally in the space $H^{s}\left(R^{+}\right)$if and only if $(\phi, h) \in \mathcal{D}_{s}^{T}$ for all $T>0$. On the other hand, it follows from our theory that for any $T>0, \mathcal{D}_{s}^{T}$ is a dense subset of the space $X_{T}^{s}$. The Baire Category Theorem thus implies that initial- and boundary-data that yield globally defined solutions comprise a dense $G_{\delta^{-}}$set in $X_{\infty}^{s}$.

Attention is turned to the case $s>3$. As pointed out earlier, if $s>7 / 2$, then $X_{T}^{s}$ is not a linear space because of the nonlinear compatibility condition imposed by membership in this class. One way to deal with this fact of life is to realize (4.1) as a specialization of a system of equations. This formulation is useful also for $3<s \leq 7 / 2$, and so it is pursued here in the entire range $s>3$.

As in Section 4, for any $s>3$, write $s=3 m+s^{\prime}$ where $m>0$ is an integer and $0<s^{\prime} \leq 3$. For $T>0$, define the space $\mathcal{Z}_{T}^{s}$ as

$$
\mathcal{Z}_{T}^{s}=Z_{T}^{3} \times Z_{T}^{3} \times \cdots \times Z_{T}^{3} \times Z_{T}^{s^{\prime}}
$$


and the space $\mathcal{X}_{T}^{s}$ as

$$
\mathcal{X}_{T}^{s}=X_{T}^{3} \times X_{T}^{3} \times \cdots X_{T}^{3} \times X_{T}^{s^{\prime}},
$$

in which there are $m$ copies of $Z_{T}^{3}$ and $X_{T}^{3}$ featured, respectively. Consider the system

$$
\left\{\begin{array}{l}
\vec{u}_{t}+\vec{u}_{x}+\vec{u}_{x x x}=-F(\vec{u})_{x}, \quad \text { for } x \geq 0,0 \leq t \leq T, \\
\vec{u}(x, 0)=\vec{\phi}(x), \quad \vec{u}(0, t)=\vec{h}(t),
\end{array}\right.
$$

where

$$
\begin{gathered}
\vec{u}=\left(u_{0}, u_{1}, \cdots, u_{m}\right)^{\tau}, \quad \vec{\phi}=\left(\phi_{0}, \phi_{1}, \cdots, \phi_{m}\right)^{\tau}, \\
\vec{h}=\left(h_{0}, h_{1}, \cdots, h_{m}\right)^{\tau}
\end{gathered}
$$

and

$$
F(\vec{u})=\frac{1}{2}\left(u_{0}^{2}, 2 u_{0} u_{1}, \cdots, \sum_{k=0}^{m}\left(\begin{array}{c}
m \\
k
\end{array}\right) u_{k} u_{m-k}\right)^{\tau},
$$

and the superscript $\tau$ connotes the transpose of the relevant vector. The pair $(\vec{\phi}, \vec{h})$ is said to be $s$-compatible if

$$
\phi_{j}(0)=h_{j}(0)
$$

for $j=0,1, \cdots, m-1$ when $s^{\prime} \leq 1 / 2$ and for $j=0,1, \cdots, m$ when $s^{\prime}>1 / 2$. By Theorem 4.8, for any $s$-compatible $(\phi, h) \in X_{T}^{s}$, the initial-boundary-value problem (4.1) has a unique solution $u \in Z_{T}^{s}$. If we let $\phi_{0}=\phi$, then $\phi_{1}, \phi_{2}, \cdots, \phi_{m}$ may be obtained recursively via (4.7). For $k=0,1, \cdots, m$, let $h_{k}=h^{(k)}$, and $u_{k}=\partial_{t}^{k} u$. If $\vec{\phi}=\left(\phi_{0}, \cdots, \phi_{m}\right), \vec{h}=\left(h_{0}, \cdots, h_{m}\right)$ and $\vec{u}=\left(u_{0}, \cdots, u_{m}\right)$, then $(\vec{\phi}, \vec{h}) \in \mathcal{X}_{T}^{s}$ and $\vec{u}$ is a solution of (6.12). In this sense, (4.1) is a special case of the system (6.12).

Theorem 6.5. Let $T>0$ and $s>3$ be given with $s=3 m+s^{\prime}$ and $0<s^{\prime} \leq 3$. Then for any s-compatible $(\vec{\phi}, \vec{h}) \in \mathcal{X}_{T}^{s}$, the system (6.12) admits a unique solution $\vec{u} \in \mathcal{Z}_{T}^{s}$.

Proof. Observe that the nonlinear system 6.12 consists of initial-boundary-value problems for $m+1$ scalar equations. Among them, the first one is the initialboundary-value problem (4.1) which only involves $u_{0}$. The second one involves only $u_{0}$ and $u_{1}$. If $u_{0}$ is considered known, then the second equation is linear in $u_{1}$, and so on. Thus we may solve the nonlinear system by solving for $u_{0}$ in the first equation, then using this determination of $u_{0}$ in the second equation and solving the corresponding linearized problem to obtain $u_{1}$ and so forth. Using Theorem 4.8 and Proposition 6.1, we obtain inductively $u_{k} \in Z_{T}^{3}$ for $k=0,1, \cdots, m-1$. The equation for $u_{m}$ has the form

$$
\left\{\begin{array}{l}
\partial_{t} u_{m}+\partial_{x} u_{m}+\partial_{x}\left(a u_{m}\right)+\partial_{x}^{3} u_{m}=f, \quad \text { for } x \geq 0,0 \leq t \leq T, \\
u_{m}(x, 0)=\phi_{m}(x), \quad u_{m}(0, t)=h_{m}(t)
\end{array}\right.
$$

where

$$
f=-\frac{1}{2} \partial_{x}\left(\sum_{k=1}^{m-1}\left(\begin{array}{c}
m \\
k
\end{array}\right) u_{k} u_{m-k}\right)
$$


and the coefficient $a=u_{0}$ is known. By Proposition 6.1, for any $s^{\prime}$-compatible $\left(\phi_{m}, h_{m}\right) \in X_{T}^{s^{\prime}}$, (6.13) possesses a unique solution $u_{m} \in Z_{T}^{s^{\prime}}$. The proof is complete.

By Theorem 6.5, for given $T>0$, the system (6.12) defines a map $\mathcal{K}_{I}$ from the space $\mathcal{X}_{T}^{s}$ to $\mathcal{Z}_{T}^{s}$ where $s=3 m+s^{\prime}$ with $m \geq 1$ and $s^{\prime} \neq 1 / 2$. The map $\mathcal{K}_{I}$ is analytic from $\mathcal{X}_{T}^{s}$ to $\mathcal{Z}_{T}^{s}$. To establish this, consider the linearized system corresponding to (6.12), namely

$$
\left\{\begin{array}{l}
\partial_{t} \vec{w}+\partial_{x} \vec{w}+\partial_{x}(J(\vec{a}) \vec{w})+\partial_{x}^{3} \vec{w}=\partial_{x} \vec{f}, \quad \text { for } x \geq 0,0 \leq t \leq T, \\
\vec{w}(x, 0)=\vec{\phi}(x), \quad \vec{w}(0, t)=\vec{h}(t),
\end{array}\right.
$$

where $J$ is the Jacobian of $F$ at $\vec{u}=\vec{a}$ given by

$$
\begin{gathered}
J(\vec{a})=\left.\frac{\partial F(\vec{u})}{\partial \vec{u}}\right|_{\vec{u}=\vec{a}}=\left(\sum_{j=0}^{k}\left(\begin{array}{c}
k \\
j
\end{array}\right)\left(\delta(i, j) a_{k-j}+a_{j} \delta(i, k-j)\right)\right)_{0 \leq k, i \leq m}, \\
\delta(i, j)= \begin{cases}1 & \text { if } i=j, \\
0 & \text { if } i \neq j,\end{cases}
\end{gathered}
$$

and

$$
\vec{f}=\left(b_{0} v_{0}, b_{1} v_{1}, \cdots, b_{m} v_{m}\right)^{\tau} .
$$

Proposition 6.6. Let $T>0$ and $s>3$ be given and let

$$
\vec{b}=\left(b_{0}, b_{1}, \cdots, b_{m}\right)^{\tau}, \quad \vec{v}=\left(v_{0}, v_{1}, \cdots, v_{m}\right)^{\tau} .
$$

Suppose $\vec{a}, \vec{b}, \vec{v} \in \mathcal{Z}_{T}^{s}$. Then for any $(\vec{\phi}, \vec{h}) \in \mathcal{X}_{T}^{s}$, 6.14) admits a unique solution $\vec{w} \in \mathcal{Z}_{T}^{s}$. Moreover,

$$
\|\vec{w}\|_{\mathcal{Z}_{T}^{s}} \leq \alpha_{T}\left(\|\vec{a}\|_{\mathcal{Z}_{T}^{s}}\right)\left(\|(\vec{\phi}, \vec{h})\|_{\mathcal{X}_{T}^{s}}+\|\vec{b}\|_{\mathcal{Z}_{T}^{s}}\|\vec{v}\|_{\mathcal{Z}_{T}^{s}}\right)
$$

where $\alpha_{T}: R^{+} \rightarrow R^{+}$is a T-dependent and continuous non-decreasing function.

Proof. The proof is very similar to that of Proposition 6.1 and is therefore omitted.

For given $\vec{u}=\mathcal{K}_{I}((\vec{\phi}, \vec{h}))$ with $(\vec{\phi}, \vec{h}) \in \mathcal{Z}_{T}^{s}$, consider the linear systems

$$
\left\{\begin{array}{l}
\partial_{t} \vec{y}_{1}+\partial_{x} \vec{y}_{1}+\partial_{x}\left(J(\vec{u}) \vec{y}_{1}\right)+\partial_{x}^{3} \vec{y}_{1}=0, \quad \text { for } x \geq 0,0 \leq t \leq T, \\
\vec{y}_{1}(x, 0)=\vec{w}_{\vec{\phi}}(x), \quad \vec{y}_{1}(0, t)=\vec{w}_{\vec{h}}(t),
\end{array}\right.
$$

and for $x \geq 0,0 \leq t \leq T$,

$$
\left\{\begin{array}{l}
\partial_{t} \vec{y}_{n}+\partial_{x} \vec{y}_{n}+\partial_{x}\left(J(\vec{u}) \vec{y}_{n}\right)+\partial_{x}^{3} \vec{y}_{n}=F_{n}\left(\vec{y}_{1}, \cdots, \vec{y}_{n-1}\right), \\
\vec{y}_{n}(x, 0)=0, \quad \vec{y}_{n}(0, t)=0
\end{array}\right.
$$

for $2 \leq n \leq N$, where

$$
F_{n}=\left(f_{n, 0}, f_{n, 1}, \cdots, f_{n, m}\right)^{\tau}
$$


with

$$
f_{n, k}=-\frac{1}{2} \partial_{x}\left(\sum_{j=0}^{k} \sum_{i=1}^{n-1}\left(\begin{array}{c}
k \\
j
\end{array}\right)\left(\begin{array}{c}
n \\
i
\end{array}\right) y_{i, j} y_{n-i, k-j}\right)
$$

for $k=0,1, \cdots, m$.

Proposition 6.7. Given $T>0, s>3$ and $\vec{g}=(\vec{\phi}, \vec{h}) \in \mathcal{X}_{T}^{s}$, let $\vec{u}=\mathcal{K}_{I}((\vec{\phi}, \vec{h}))$. Then the system de.15)- 6.16) defines a homogeneous polynomial $\mathcal{K}_{I}^{(N)}(\vec{g})\left[\vec{w}^{n}\right]$ of degree $n$ from $\mathcal{X}_{T}^{s}$ to $\mathcal{Z}_{T}^{s}$. Moreover, there exists a constant $C$ such that

$$
\left\|\vec{y}_{n}\right\|_{\mathcal{Z}_{T}^{s}} \leq C^{n} n !\|\vec{w}\|_{\mathcal{X}_{T}^{s}}^{n}
$$

for any $n \geq 2$, where $C=C\left(T,\|\vec{u}\|_{\mathcal{Z}_{T}^{s}}\right)$, and it may be that $C \rightarrow+\infty$ as $T \rightarrow \infty$ or $\|\vec{u}\|_{\mathcal{Z}_{T}^{s}} \rightarrow \infty$, but in any case $C \rightarrow 0$ if $\|\vec{u}\|_{\mathcal{Z}_{T}^{s}} \rightarrow 0$ or if $T \rightarrow 0$.

Proof. This follows from Proposition 6.6 by direct computation.

For $\vec{w} \in \mathcal{X}_{T}^{s}$, define a Taylor polynomial $P_{N}(\vec{w})$ of degree $n$ by

$$
P_{n}(\vec{w})=\sum_{k=0}^{N} \frac{\mathcal{K}_{I}^{(k)}(g)\left[\vec{w}^{k}\right]}{k !}=\mathcal{K}_{I}(\vec{g})+\sum_{k=1}^{N} \frac{\vec{y}_{k}}{k !},
$$

and a formal Taylor series by

$$
P(\vec{w})=\sum_{k=0}^{\infty} \frac{\mathcal{K}_{I}^{(k)}(\vec{g})\left[\vec{w}^{k}\right]}{k !} .
$$

Arguing as in the proof of Proposition 6.3 gives the following result.

Proposition 6.8. For any $\vec{g}=(\vec{\phi}, \vec{h}) \in \mathcal{D}_{s}^{T}=\mathcal{D}_{s}^{T}\left(\mathcal{K}_{I}\right)$, there exists an $\eta>0$ depending only on $\left\|\mathcal{K}_{I}(\vec{g})\right\|_{\mathcal{Z}_{T}^{s}}$ such that the formal Taylor series (6.18) is uniformly convergent in the space $\mathcal{Z}_{T}^{s}$ for $\vec{w} \in \mathcal{X}_{T}^{s}$ with $\|\vec{w}\|_{\mathcal{X}_{T}^{s}} \leq \eta$. Moreover, if $\vec{v}=P(\vec{w})$, then $\vec{v} \in \mathcal{Z}_{T}^{s}$ solves the problem

$$
\left\{\begin{array}{l}
\partial_{t} \vec{v}+\partial_{x} \vec{v}+\partial_{x}(F(\vec{v}) \vec{v})+\partial_{x}^{3} \vec{v}=0, \quad \text { for } x \geq 0,0 \leq t \leq T, \\
\vec{v}(x, 0)=\vec{\phi}+\vec{w}_{\vec{\phi}}, \quad \vec{v}(0, t)=\vec{h}+\vec{w}_{\vec{h}}
\end{array}\right.
$$

for $0 \leq t \leq T$.

As a direct consequence of Proposition 6.8, we arrive at the following satisfactory result.

Theorem 6.9 (Analyticity). For any $T>0$ and $s>3$ the nonlinear problem (6.12) establishes a map $\mathcal{K}_{I}$ from the space $\mathcal{X}_{T}^{s}$ to the space $\mathcal{Z}_{T}^{s}$. The map $\mathcal{K}_{I}$ is analytic from $\mathcal{X}_{T}^{s}$ to $\mathcal{Z}_{T}^{s}$ in the sense that for any $\vec{\phi} \in \mathcal{X}_{T}^{s}$, there exists an $\eta>0$ such that for any $\vec{h} \in \mathcal{X}_{T}^{s}$ with $\|\vec{h}\|_{\mathcal{X}_{T}^{s}} \leq \eta$, the Taylor series expansion

$$
\mathcal{K}_{I}(\vec{\phi}+\vec{h})=\sum_{n=0}^{\infty} \frac{\mathcal{K}_{I}^{(n)}(\phi)\left[\vec{h}^{n}\right]}{n !}
$$

converges in the space $\mathcal{Z}_{T}^{s}$. Moreover, the convergence is uniform with regard to $h$ in the aforementioned ball in $\mathcal{X}_{T}^{s}$. 


\section{REFERENCES}

1. T. B. Benjamin, J. L. Bona and J. J. Mahony, Model equations for long waves in nonlinear, dispersive media, Philos. Trans. Royal Soc. London Series A 272 (1972), 47-78. MR 55:898

2. J. L. Bona and P. J. Bryant, A mathematical model for long waves generated by wave makers in nonlinear dispersive systems, Proc. Cambridge Philos. Soc. 73 (1973), 391-405. MR 49:4409

3. J. L. Bona and M. Chen, A Boussinesq system for two-way propagation of nonlinear dispersive waves, Phys. D 116 (1998), 191-224. MR 99f:76021

4. J. L. Bona, M. Chen and J.-C. Saut, Boussinesq equations and other systems for smallamplitude long waves in nonlinear dispersive media, Part I. Derivation and the linear theory. To appear.

5. J. L. Bona and L. Luo, Initial-boundary-value problems for model equations for the propagation of long waves, Evolution Equations, Lecture Notes in Pure and Applied Mathematics, vol. 168 (G. Ferreyra, G. Goldstein \& F. Neubrander, ed.), Dekker, New York, 1995, pp. 65-94. MR 95i:35151

6. J. L. Bona and L. Luo, Generalized Korteweg-de Vries equation in a quarter plane, Contemporary Math. 221 (1999), 59-125. MR 99m:35201

7. J. L. Bona, W. G. Pritchard and L. R. Scott, An evaluation of a model equation for water waves, Philos. Trans. Royal Soc. London Series A 302 (1981), 457-510. MR 83a:35088

8. J. L. Bona and L. R. Scott, Solutions of the Korteweg-de Vries equation in fractional order Sobolev spaces, Duke Math. J. 43 (1976), 87-99. MR 52:14694

9. J. L. Bona and R. Smith, The initial-value problem for the Korteweg-de Vries equation, Philos. Trans. Royal Soc. London Series A 278 (1975), 555-601. MR 52:6219

10. J. L. Bona and R. Winther, The Korteweg-de Vries equation posed in a quarter plane, SIAM J. Math. Anal. 14 (1983), 1056-1106. MR 85c:35076

11. J. L. Bona and R. Winther, Korteweg-de Vries equation in a quarter plane, continuous dependence results, Diff. and Integral Equ., 2 (1989), 228-250. MR 90e:35134

12. J. L. Bona and B.-Y. Zhang, The initial-value problem for the forced Korteweg-de Vries equation, Proc. Royal Soc. Edinburgh Section A, 126 (1996), 571-598. MR 97j:35133

13. J. Bourgain, Fourier transform restriction phenomena for certain lattice subsets and applications to non-linear evolution equations, part I: Schrödinger equations, Geom. \& Funct. Anal. 3 (1993), 107-156. MR 95d:35160a

14. J. Bourgain, Fourier transform restriction phenomena for certain lattice subsets and applications to non-linear evolution equations, part II: the KdV equation, Geom. \& Funct. Anal. 3 (1993), 209-262. MR 95d:35160b

15. B. A. Bubnov, Solvability in the large of nonlinear boundary-value problem for the Kortewegde Vries equations, Differential Equations 16 (1980), 24-30. MR 81e:35111

16. A. Cohen, Solutions of the Korteweg-de Vries equation from irregular data, Duke Math. J. 45 (1978), 149-181. MR 57:10283

17. A. Cohen, Existence and regularity for solutions of the Korteweg-de Vries equation, Arch. Rat. Mech. Anal. 71 (1979), 143-175. MR 80g:35109

18. T. Colin and J.-M. Ghidaglia, An initial-boundary-value problem for the Korteweg-de Vries equation posed on a finite interval, to appear in Adv. Diff. Eq..

19. P. Constantin and J.-C. Saut, Local smoothing properties of dispersive equations, J. American Math. Soc. 1 (1988), 413-446. MR 89d:35150

20. W. Craig, T. Kappeler and W. A. Strauss, Gain of regularity for equations of the Korteweg-de Vries type, Ann. Inst. Henri Poincaré 9 (1992), 147-186. MR 93j:35153

21. T. E. Dushane, On existence and uniqueness for a new class of nonlinear partial differential equations using compactness and differential-difference schemes, Trans. Amer. Math. Soc. 188 (1974), 77-96. MR 49:3349

22. A. V. Faminskii, The Cauchy problem and the mixed problem in the half strip for equations of Korteweg-de Vries type, (Russian) Dinamika Sploshn. Sredy 63 (1983), 152-158. MR 87c:35137

23. A. V. Faminskii, A mixed problem in a semistrip for the Korteweg-de Vries equation and its generalizations, (Russian) Dinamika Sploshn. Sredy 258 (1988), 54-94.

24. A. S. Fokas and B. Pelloni, The solution of certain initial boundary-value problems for the linearized Korteweg-de Vries equation, Proc. Royal. Soc. London Series A 454 (1998), 645657. MR 99e:35197 
25. A. S. Fokas and A. R. Its, Integrable equations on the half-infinite line. Solitons in science and engineering: theory and applications, Chaos Solitons Fractals 5 (1995), 2367-2376. MR 96i:35109

26. A. S. Fokas and A. R. Its, An initial-boundary value problem for the Korteweg-de Vries equation, In the proceedings of the conference: Solitons, nonlinear wave equations and computation (New Brunswick, NJ, 1992), Math. Comput. Simulation 37 (1994), 293-321. MR 95m:35162

27. A. S. Fokas and A. R. Its, Soliton generation for initial-boundary value problems, Phys. Rev. Lett. 68 (1992), 3117-3120. MR 93a:35130

28. J. Ginibre and Y. Tsutsumi, Uniqueness for the generalized Korteweg-de Vries equations, SIAM J. Math. Anal. 20 (1989), 1388-1425. MR 90i:35240

29. J. Ginibre, Y. Tsutsumi and G. Velo, Existence and uniqueness of solutions for the generalized Korteweg de Vries equation, Math. Z. 203 (1990), 9-36. MR 90m:35168

30. J. Ginibre and G. Velo, Smoothing properties and retarded estimates for some dispersive evolution equations, Comm. Math. Phys. 144 (1992), 163-188. MR 93a:35065

31. G. H. Hardy, J. E. Littlewood and G. Pólya, Inequalities, Cambridge University Press, Cambridge, 1934. (2nd ed., 1952, MR 13:727e)

32. J. L. Hammack, A note on tsunamis: their generation and propagation in an ocean of uniform depth, J. Fluid Mech. 60 (1973), 769-799.

33. J. L. Hammack and H. Segur, The Korteweg-de Vries equation and water waves, Part 2. Comparison with experiments, J. Fluid Mech. 65 (1974), 289-313. MR 51:2446

34. Y. Kametaka, Korteweg-de Vries equation $I-I V$, Proc. Japan Acad. 45 (1969), 552-558 \& 656-665. MR 40:6043. MR 40:6044 MR 41:7311; MR 41:7312

35. T. Kato, Quasilinear equations of evolution, with applications to partial differential equations, Springer Lecture Notes in Math. 448 (1975), 27-50.

36. T. Kato, On the Korteweg-de Vries equation, Manuscripta Math. 28 (1979), 89-99. MR 80d:35128

37. T. Kato, The Cauchy problem for the Korteweg-de Vries equation, Pitman Research Notes in Math. 53 (1981), 293-307. MR 82m:35129

38. T. Kato, On the Cauchy problem for the (generalized) Korteweg-de Vries equations, Advances in Mathematics Supplementary Studies, Studies in Applied Math. 8 (1983), 93-128. MR 86f:35160

39. C. E. Kenig, G. Ponce and L. Vega, On the (generalized) Korteweg-de Vries equation, Duke Math. J. 59 (1989), 585-610. MR 91d:35190

40. C. E. Kenig, G. Ponce and L. Vega, Oscillatory integrals and regularity of dispersive equations, Indiana Univ. Math. J. 40 (1991), 33-69. MR 92d:35081

41. C. E. Kenig, G. Ponce and L. Vega, Well-posedness of the initial value problem for the KdV equation, J. American Math. Soc. 4 (1991), 323-347. MR 92c:35106

42. C. E. Kenig, G. Ponce and L. Vega, Well-posedness and scattering results for the generalized Korteweg-de Vries equations via the contraction principle, Comm. Pure Appl. Math. 46 (1993), 527-620. MR 94h:35229

43. C. E. Kenig, G. Ponce and L. Vega, The Cauchy problem for the Korteweg-de Vries equation in Sobolev spaces of negative indices, Duke Math. J. 71 (1993), 1-21. MR 94g:35196

44. C. E. Kenig, G. Ponce and L. Vega, A bilinear estimate with applications to the KdV equation, J. American Math. Soc. 9 (1996), 573-603. MR 96k:35159

45. S. N. Kruzhkov and A. V. Faminskii, Generalized solutions of the Korteweg-de Vries equation, (Russian) Dokl. Akad. Nauk SSSR 261 (1981), 1296-1298. MR 83f:35098

46. S. N. Kruzhkov and A. V. Faminskii, Generalized solutions of the Cauchy problem for the Korteweg-de Vries equation, (in Russian) Mat. Sb. (N.S.) 120 (1983), 396-425. MR 85c:35079 English translation in Math USSR Sbornik 48 (1984), 391-421.

47. J.-L. Lion and E. Magenes, Non-homogeneous boundary value problems and applications, Vol. 1, Springer-Verlag, Heidelberg, 1972. MR 50:2670

48. R. M. Miura, The Korteweg-de Vries equation: A survey of results, SIAM Review 18 (1976), 412-459. MR 53:8689

49. D. L. Russell and B.-Y. Zhang, Smoothing and decay properties of solutions of the Kortewegde Vries equation on a periodic domain with point dissipation, J. Math. Anal. Appl., 190 (1995), 449-488. MR 95k:35180

50. R. L. Sachs, Classical solutions of the Korteweg-de Vries equation for non-smooth initial data via inverse scattering, Comm. P.D.E. 10 (1985), 29-89. MR 86h:35126 
51. J.-C. Saut, Applications de l'interpolation non linéaire a des problèmes d'evolution nonlineaire, J. Math. Pures Appl. 54 (1975), 27-52. MR 56:12625

52. J.-C. Saut and R. Temam, Remarks on the Korteweg-de Vries equation, Israel J. Math. 24 (1976), 78-87. MR 56:12676

53. A. Sjöberg, On the Korteweg-de Vries equation: Existence and uniqueness, J. Math. Anal. Appl. 29 (1970), 569-579. MR 53:13885

54. P. Sjölin, Regularity of solutions to the Schrödinger equation, Duke Math. J. 55 (1987), 699715. MR 88j:35026

55. E. M. Stein and G. Weiss, Introduction to Fourier Analysis on Euclidean Spaces, Princeton University Press, Princeton 1971. MR 46:4102

56. S. M. Sun, The Korteweg-de Vries equation on a periodic domain with singular-point dissipation, SIAM J. Control Optim. 34 (1996), 892-912. MR 97a:35208

57. L. Tartar, Interpolation non linèaire et régularité, J. Funct. Anal. 9 (1972), 469-489. MR 46:9717

58. R. Temam, Sur un problème non linéaire, J. Math. Pures Appl. 48 (1969), 159-172. MR 41:5799

59. M. M. Tom, Smoothing properties of some weak solutions of the Benjamin-Ono equation, Diff. \& Integral Equ. 3 (1990), 683-694. MR 91e:35191

60. Y. Tsutsumi, The Cauchy problem for the Korteweg-de Vries equation with measures as initial data, SIAM J. Math. Anal. 20 (1989), 582-588. MR 90g:35153

61. L. Vega, Schrödinger equations: pointwise convergence to the initial data, Proc. American Math. Soc. 102 (1988), 874-878. MR 89d:35046

62. N. J. Zabusky and C. J. Galvin, Shallow-water waves, the Korteweg-de Vries equation and solitons, J. Fluid Mech. 47 (1971), 811-824.

63. B.-Y. Zhang, Boundary stabilization of the Korteweg-de Vries equations, in the Proc. of International Conference on Control and Estimation of Distributed Parameter Systems: Nonlinear Phenomena held in Vorau (Styria, Austria), July 18-24, 1993, International Series of Numerical Mathematics 118 (1994), 371-389. MR 95j:93037

64. B.-Y. Zhang, Taylor series expansion for solutions of the KdV equation with respect to their initial values, J. Funct. Anal. 129 (1995), 293-324. MR 96a:35016

65. B.-Y. Zhang, Analyticity of solutions for the generalized Korteweg-de Vries equation with respect to their initial datum, SIAM J. Math. Anal. 26 (1995), 1488-1513. MR 97a:35210

66. B.-Y. Zhang, A remark on the Cauchy problem for the Korteweg-de Vries equation on a periodic domain, Diff. and Integral Equ. 8 (1995), 1191-1204. MR 96a:35183

Department of Mathematics, Texas Institute for Computational and Applied Mathematics, University of Texas, Austin, Texas 78712

Current address: Department of Mathematics, Statistics and Computer Science, University of Illinois at Chicago, Chicago, Illinois 60607

E-mail address: bona@math.utexas.edu

Department of Mathematics, Virginia Polytechnic Institute and State University, BlaCKSBURG, ViRginia 24061

E-mail address: sun@math.vt.edu

Department of Mathematics, University of Cincinnati, Cincinnati, Ohio 45221

E-mail address: bzhang@math.uc.edu 